\title{
Towards Power
}

\section{Autonomous Wireless \\ Sensors}

Colm Mc Caffrey

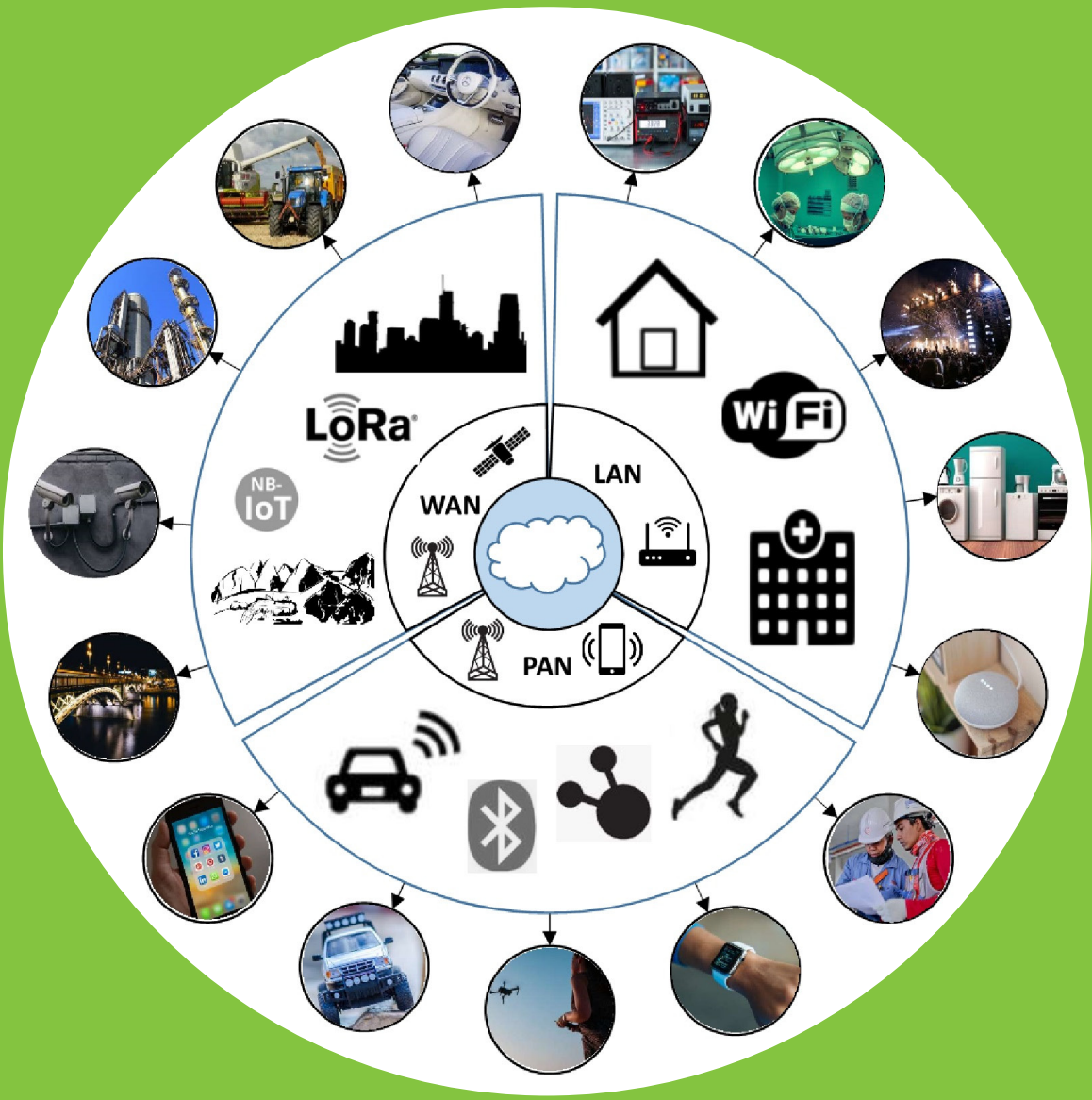




\title{
Towards Power Autonomous Wireless Sensors
}

\author{
Colm Mc Caffrey
}

A doctoral dissertation completed for the degree of Doctor of Science (Technology) to be defended, with the permission of the Aalto University School of Electrical Engineering, at a public examination held at Large Seminar Hall, Micronova, Tietotie 3 of the school on 2nd August 2019 at 12:00 
Prof. D.Sc. (Tech.) Ville Viikari, Aalto University, Finland

\section{Thesis advisor}

D. Sc. (Tech.) Pekka Pursula, VTT - Technical Research Center of Finland

\section{Preliminary examiners}

Prof. Ph.D. Anja Skrivervik, Ecole polytechnique fédérale de Lausanne, Switzerland

Prof. Ph.D. Yvan Duroc, Lyon University, France

\section{Opponent}

Prof. Ph.D. Gaetano Marrocco, University of Roma Tor Vergata, Italy

DOCTORAL DISSERTATIONS 130/2019

(C) 2019 Colm Mc Caffrey

ISBN 978-952-60-8639-2 (printed)

ISBN 978-952-60-8640-8 (pdf)

ISSN 1799-4934 (printed)

ISSN 1799-4942 (pdf)

http://urn.fi/URN:ISBN:978-952-60-8640-8

Unigrafia Oy

Helsinki 2019

Finland 
Author

Colm Mc Caffrey

Name of the doctoral dissertation

Towards Power Autonomous Wireless Sensors

\begin{tabular}{|c|c|}
\hline \multicolumn{2}{|l|}{ Unit Electronics and Nanoengineering } \\
\hline \multicolumn{2}{|c|}{ Series Aalto University publication series DOCTORAL DISSERTATIONS 130/2019 } \\
\hline \multicolumn{2}{|l|}{ Field of research Radio Engineering } \\
\hline \multicolumn{2}{|l|}{ Manuscript submitted 20 June 2019} \\
\hline \multicolumn{2}{|l|}{ Permission for public defence granted (date) 17 May 2019} \\
\hline$\bigotimes$ Article dissertation & Essay dissertation \\
\hline
\end{tabular}

\section{Abstract}

To enable widespread deployment of sensor systems in our world, they must be wireless and power autonomous. There is no universal solution to achieve such a device. Instead a system level approach must be taken that considers the sensor, its readout, wireless communications and powering methods available in the context of the application requirements and sensor system lifetime. Achieving power autonomy requires a two front approach; addressing both the need and the supply. The optimisation of power consumption in this work focuses on the radio communications, highlighted as one of the most power critical subsystems. A power-optimised system enables the exploitation of application appropriate energy sources; whether they be battery power in the case of short lifetime devices, energy harvesting where reliable sources exist, wireless power transfer where it can be applied, or passive radio backscattering techniques.

This thesis presents four independent systems, each a power autonomous solution in the context of its application requirements. The first system integrates a voltammetric electronic tongue sensor for gastrointestinal disease diagnosis, with power provided by a high-density lithium manganese dioxide primary cell, ample for the 72 hours operation time of the capsule. The capsule contains an electrically small loop antenna and communicates wirelessly at $433 \mathrm{MHz}$ to a remote receiver. The second system applies a MEMS acoustic emission sensor for online condition monitoring of valve leakage in the petrochemical industry, utilising thermal energy harvesting in the sensor node, and industrial current loop harvesting in the gateway to achieve power autonomy for continuous operation. The device communicates at $2.45 \mathrm{GHz}$ and includes an ultra-low power wake-up radio based on passive down converting receiver to enable the sensor to be in an ultra-low power mode and asynchronously interrogated by the gateway.

The third system exploits resonant inductive coupling for the actuation of a wirelessly powered soft robotic caterpillar which itself is potentially a fully passive wireless sensor platform. The system utilises a frequency sweeping power transmission mechanism, around 8.5 $\mathrm{MHz}$, to wirelessly transfer energy to multiple shape memory alloy actuators, enabling the generation of a wave of force through the caterpillar body providing locomotion. The fourth system employs a fully passive wireless sensing platform based on radio backscattering at UHF frequencies (around $868 \mathrm{MHz}$ ), and a harmonic resonant sensor based on the third order intermodulation principle.

In its entirety, this work represents progress on multiple fronts on the key challenge of powering the next generation of IoT devices.

Keywords Wireless sensors, IoT, RF communication, power autonomy, systems engineering, energy harvesting, wireless power transfer, passive wireless sensors

\begin{tabular}{lc}
\hline ISBN (printed) $978-952-60-8639-2$ & ISBN (pdf) $978-952-60-8640-8$ \\
\hline ISSN (printed) $1799-4934$ & ISSN (pdf) $1799-4942$ \\
\hline Location of publisher Helsinki & Location of printing Helsinki Year 2019 \\
\hline Pages 186 & urn http://urn.fi/URN:ISBN:978-952-60-8640-8 \\
\hline
\end{tabular}



Dedicated to the memory of my father

Vincent Mc Caffrey 



\section{Acknowledgements}

The work of this Thesis was conducted in association with Aalto University School of Electrical Engineering. The efforts were primarily undertaken at VTT - Technical Research Centre of Finland, with auxiliary efforts at Tyndall National Institute, Ireland and The University of Tokyo, Japan.

I would like to express gratitude for all funding sources that contributed towards this work including; the VTT Thesis completion grant, the EU ERASMUS Mundus TEAM exchange program, TEKES SHOK Intersync project and Enterprise Ireland DIACAPS project.

I would like to express sincerest gratitude to my Thesis Supervisor, Prof. Ville Viikari for his support and encouragement throughout the Doctoral studies and as a key contributor to the core ideas of both the Zero Power Sensor system, and the ultra-low power wake up radio used in this work.

I would like to especially acknowledge the mentorship and guidance of my Thesis Mentor and Research Team Leader, Dr. Pekka Pursula. His positivity, flexibility, patience, understanding, technical excellence and leadership continue to be an inspiration to me.

I would like to express gratitude to my colleagues at VTT, most especially Dr. Nadine Pesonen, for her mentorship in the early stages of the thesis and her positive and proactive approach to research. In addition, I would acknowledge Dr Jacek Flak as one of my closest co-authors, Juha-Matti Saari, as key technical contributors to this work. Further I would like to mention Seniors and Mentors at VTT including, Dr. Kai Nummila, Dr. Kaarle Jaakkola, Dr. Tomi Mattila and peers Jinsong, Tapio, Samuli, Mohammad, and Teuvo.

I would like to acknowledge Yoshiro Kawahara Sensei, and Takuya Umedachi Sensei, as supervising professors for the robotic caterpillar work at the University of Tokyo, and colleagues and contributors Narusue-san, Sasatani-san and Weiwei Jiang. Along with Eren, Sasha, Yoshie, Christian and Paul; friends in the Tokyo adventure. 
I would like to acknowledge the revolutionary work of the great Nicola Tesla, father of modern power systems, radio communications and wireless power transfer. His ideas are at the core of this work.

I would like to acknowledge Dr. Vladimir Ogurtsov as supervising professor in the swallowable capsule work. Dr. Karen Twomey as principle investigator and colleagues and contributors Dr. Olivier Chevalerias, Dr. Pio Jesudoss and Dr. Eva Alvarez. Along with friends at Tyndall including Padraig, Claire, Brian, Ran, Pierre and Jeff.

I would like to express gratitude to friends in the VTT Young Professionals, especially Caitlin, Visa, Tiia, Neils, Riina, Kari and Petri, and the Young European Associated Researchers especially Clara, Alexis, Tiia and Jef.

For there is more to life than work, I would like to acknowledge friends, Tuomo, Kata, Jason, Antti, Viljami, Michael, Kaarla, Zubi, Joppe, Antonio and Riikka and those too numerous to mention individually, in the Shoto RY Karate Club Helsinki, Finnish Rugby Referees Association, Helsingin Aikuisopisto Guitar courses and Finnish language courses, the International English Speakers Association of Finland, the Helsinki Couchsurfing community, UCC Karate Club, UCC International Students Society, UCC Skydive Club, Tyndall Postgraduate Student Committee.

I would like to express my deepest gratitude to my mother, Maura, for trusting me on my lifelong journey and giving me the most important things a parent ever can - roots and wings. Also to my siblings Tara, Ciara, Vincent and Clodagh.

I would, for a second time, sincerely thank my sister Ciara for kindly proof reading this Thesis along with so many of my previous works.

Finally, I would like to express sincere gratitude to Liis. You ground me and remind me what is important in life. Thank you for filling the last year of my studies with joy.

Helsinki, 20 ${ }^{\text {th }}$ June 2019

Colm Mc Caffrey 


\section{Contents}

List of Abbreviations........................................................................... 7

List of Symbols .............................................................................. 9

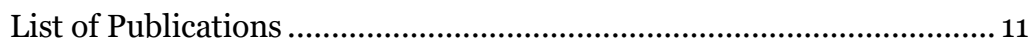

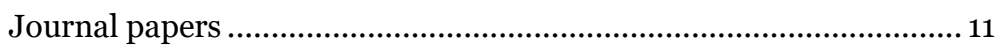

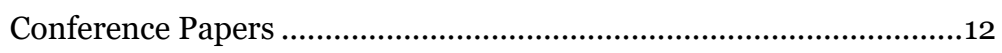

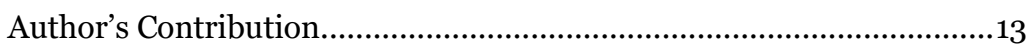

1. Introduction........................................................................17

1.1 Research Problem ................................................................ 17

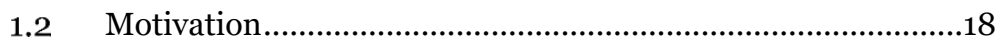

$1.3 \quad$ Scientific Contribution.........................................................21

2. Background and State of the Art ............................................... 23

2.1 Wireless Sensor System Architecture ..................................... 23

2.2 Power Consumption Overview ............................................. 24

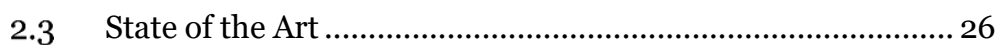

2.3.1 Power Consumption Optimisation of Wireless Sensors ..... 26

2.3.2 Ultra-Low Power Wireless Communications ...................... 27

2.3.3 Energy Storage for IoT Sensors......................................... 28

2.3.4 Energy Harvesting Sensor Systems................................... 29

2.3.5 Wireless Power Transfer .....................................................31

2.3.6 Passive Wireless Sensors ................................................... 32

2.3.7 Swallowable Capsules........................................................ 33

3. Wireless Sensor Systems ......................................................... 35

3.1 Swallowable E-tongue Capsule ............................................... 35

3.1.1 Requirements and Constraints ............................................ 36

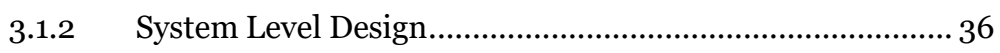


3.1.3 E-tongue Sensor and Analogue Front End .........................37

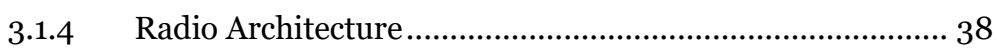

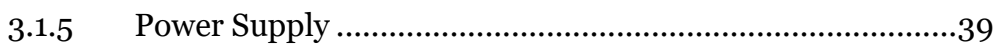

3.1.6 Test and Evaluation .......................................................39

3.1.7 Summary ........................................................................42

3.2 Power Autonomous Condition Monitoring .............................43

3.2.1 Requirements and Constraints .........................................44

3.2.2 System Level Design .........................................................44

3.2.3 Acoustic Emission Sensor ..................................................45

3.2.4 Radio Architecture .............................................................46

3.2.5 Power Supply ....................................................................4

3.2.6 Test and Evaluation ..........................................................49

3.2.7 Summary .................................................................... 52

3.3 Wirelessly Powered Soft Robot .............................................5 55

3.3.1 Requirements and Constraints .........................................56

3.3.2 System Level Design .....................................................56

3.3.3 Wireless Power Transfer ................................................... 57

3.3.4 Test and Evaluation .......................................................59

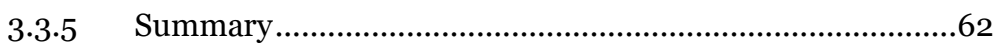

3.4 Passive Wireless Sensing ....................................................63

3.4.1 Requirements and Constraints ..........................................64

3.4.2 Radio Architecture ..........................................................64

3.4.3 Reader Integration..........................................................67

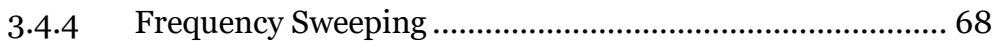

3.4.5 Activation and Identification ..............................................69

3.4.6 Test and Evaluation ........................................................ 70

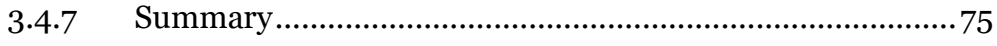

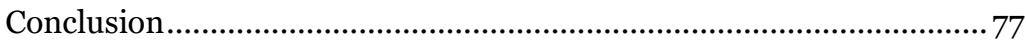

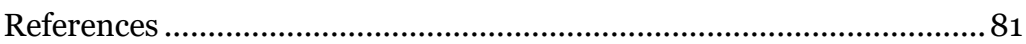

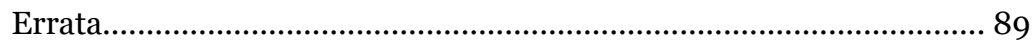

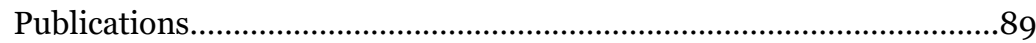




\section{List of Abbreviations}

3/4/5G Third / fourth / fifth generation (mobile network)

ADC Analogue to digital converter

ANT Proprietary 2.4 GHz low power radio standard

ASIC Application specific integrated circuit

ATEX Appareils destinés à être utilisés en ATmosphères EXplosives

BLE Bluetooth low energy

CE Counter electrode

DSB Dual side band

COTS Commercial off-the-shelf

EPC Electronic product code

FDMA Frequency division multiple access

FSK $\quad$ Frequency shift keying

GI Gastrointestinal (tract)

HFSS (Ansys) High frequency structure simulator

IoT Internet of things

ISM Industrial, Scientific and Medical (radio communication bands)

LAN Local area network

LoRa Low-power long-range (radio communication protocol)

LTE Long term evolution (mobile network)

MCU Microcontroller unit

MEMS Microelectromechanical System

MMAC Million multiples and accumulations

NB-IoT Narrow band internet of things

NFC Near field communication 
PAN Personal area network

PCB Printed circuit board

PEEK Polyether ether ketone

PER Packet error rate

PLL Phase locked loop

PWM Pulse width modulated

RE Reference electrode

RF Radio frequency

RFID Radio frequency identification

RMS Root mean squared

RSSI Received signal strength

SAW Surface acoustic wave

SRF Self resonant frequency

SMA Shape memory alloy (not to be confused with subminiture-A)

SNR Signal to noise ratio

SOI Silicon on insulator

SSB Single side band

TDMA Time division multiple access

TEG Thermoelectric generator

UART Universal asynchronous receiver transmitter

UHF Ultra high frequency

USB Universal serial bus

VCO Voltage controller oscillator

VNA Vector network analyser

WPT Wireless Power Transfer

WSN Wireless sensor network

WAN Wide area network

WE Working electrode

Wi-Fi Wireless fidelity (trademark meaning IEEE 802.11x)

WISP Wireless identification and sensing platform 


\section{List of Symbols}

\begin{tabular}{|c|c|}
\hline$C_{n}$ & Capacitance (related to $\mathrm{n}^{\text {th }}$ circuit) \\
\hline$f_{1,} f_{2}$ & UHF carrier frequencies \\
\hline$f_{\mathrm{d}}$ & Difference frequency $\left(f_{1}-f_{2}\right)$ \\
\hline$f_{\mathrm{LO}}$ & Local oscillator frequency \\
\hline$f_{\mathrm{IF}}$ & Intermediate frequency \\
\hline$F_{\mathrm{R}}$ & Resonant frequency \\
\hline$G_{\mathrm{tx}}$ & Transmitter antenna gain \\
\hline$G_{\text {tag }}$ & Sensor tag antenna gain \\
\hline$I, Q$ & Baseband demodulated quadrature signals \\
\hline$I_{\mathrm{HI}}, I_{\mathrm{LO}}$ & Intermediate frequency quadrature signals (sine) \\
\hline$I_{\mathrm{CELL}}$ & Electrochemical cell current \\
\hline$K_{n}$ & $\begin{array}{l}\text { Coupling coefficient between transmitter and } \mathrm{n}^{\text {th }} \text { re- } \\
\text { ceiver }\end{array}$ \\
\hline$K_{x y}$ & Coupling coefficient between $\mathrm{x}^{\text {th }}$ and $\mathrm{y}^{\text {th }}$ coil \\
\hline$L_{n}$ & Inductance (related to $\mathrm{n}^{\text {th }}$ circuit) \\
\hline$P_{\mathrm{MAX}}$ & Maximum harvested power \\
\hline$P_{\text {MEAS }}$ & Measured harvested power \\
\hline$P_{\mathrm{N}}$ & Noise power \\
\hline$P_{\mathrm{tx}}$ & Transmitted power \\
\hline$Q_{\mathrm{HI}}, Q_{\mathrm{LO}}$ & Intermediate frequency quadrature signals (cosine) \\
\hline$R_{n}$ & Resistance (related to $\mathrm{n}^{\text {th }}$ circuit) \\
\hline$S_{3}$ & $3^{\text {rd }}$ order intermodulation conversion loss \\
\hline$V_{\text {bias }}$ & Bias voltage \\
\hline$V_{\text {out }}$ & Output voltage \\
\hline$V_{\mathrm{REF}}$ & Reference voltage \\
\hline
\end{tabular}




\section{List of Publications}

This doctoral dissertation consists of a summary of the following publications which are referred to in the text by their numerals.

\section{Journal papers}

I. C. Mc Caffrey, O. Chevalerias, C. O'Mathuna, and K. Twomey, "Swallowable-capsule technology", IEEE Pervasive Computing, vol. 7, no. 1, pp. 23-29, January 2008.

II. C. Mc Caffrey, K. Twomey, and V.I. Ogurtsov, "Development of a wireless swallowable capsule with potentiostatic electrochemical sensor for gastrointestinal track investigation”, Sensors and Actuators B: Chemical, vol. 218, pp. 815, October 2015.

III. C. Mc Caffrey, T. Sillanpää, H. Huovila, J. Nikunen, S. Hakulinen, and P. Pursula, "Energy autonomous wireless valve leakage monitoring system with acoustic emission sensor," IEEE Transactions on Circuits and Systems I: Regular Papers, vol. 64, no. 11, pp. 2884-2893, Nov. 2017.

IV. C. Mc Caffrey, T. Umedachi, J. Weiwei, T. Sasatani, R. Niiyama, T. Narusue, and Y. Kawahara, "Soft robotic caterpillar with wirelessly powered shape memory alloy actuators", manuscript under review for publication in the Journal of Soft Robotics.

V. C. Mc Caffrey, J. Flak, and P. Pursula, "Development and optimisation of a reader for fully passive wireless sensors", manuscript under review for publication for publication in the IEEE Transactions on Instrumentation and Measurement. 


\section{Conference Papers}

VI. C. Mc Caffrey, O. Chevalerias, E. Leyraud, K. Twomey, and V. I. Ogurtsov, "RF communications for a swallowable capsule," presented at Royal Irish Academy Research Colloquium Emerging Trends in Wireless Communication, Dublin, April 24th 2008.

VII. C. Mc Caffrey, J. Doyle, V. I. Ogurtsov, K. Twomey, and D.W.M. Arrigan, "Development and evaluation of an on-chip potentiostat for biomedical applications," in Proc. Of the Third int. conf. on biomedical electronics and devices, BIODEVICES 2010, Valencia, Spain, 19-23 Jan 2010, pp 103 - 107.

VIII.C. M. Caffrey, N. Pesonen and P. Pursula, "Activation and identification of fully passive wireless sensors," in 2016 IEEE Sensors, Orlando, FL, USA, 30 Oct - 2 Nov 2016, pp. 1-3.

IX. C. M. Caffrey, J. Flak, I. Marttila, N. Pesonen and P. Pursula, "Development of a reader device for fully passive wireless sensors," in 2017 IEEE Sensors, Glasgow, UK, 30 Oct - 1 Nov 2017, pp. 1-1. 


\section{Author's Contribution}

Publication 1: Swallowable capsule technology

The candidate was the leading author, responsible for the analysis of the literature and state of the art, and the drafting, submission and review of the paper. With guidance from the supervising professors, the candidate conducted the extensive review of published research literature and commercial activities on the topic including communicating with the relevant stakeholders in research projects and companies. The candidate produced all of the text, and worked with supervisors to complete the draft to a high standard. The candidate submitted the paper, and took responsibility for the improvements in response to the reviewer feedback. The candidate is the corresponding author.

Publication 2: Development of a wireless swallowable capsule with potentiostatic electrochemical sensor for gastrointestinal track investigation

The candidate was the leading author, producing the majority of the text and responsible for the completion of the draft. With guidance from the supervising professor, the candidate was the main technical contributor to the design, implementation and evaluation of the swallowable electronic system, including the potentiostat circuit, signal processing circuit, microcontroller and power supply unit. The candidate worked on the design and integration of the radio communication and receiver module. The candidate directly produced all the paper's results, with the exception of the sensor characterisation in faecal samples which was carried out by expert co-authors. The candidate worked closely with medical, chemical and packaging partners for the integration of the device, and the system characterisation. The candidate supported the corresponding author in the submission and the improvements based on the reviewer comments.

Publication 3: Energy autonomous wireless valve leakage monitoring system with acoustic emission sensor

The candidate was the leading author, producing the majority of the text and coordinating the contributing authors' input. The candidate was the main technical contributor to the design, implementation, evaluation and deployment of the system. The candidate was responsible for the implementation, test and evaluation of the wake up radio technology at the core of the device, and production of the radio results. The candidate designed and implemented the cur- 
rent loop energy harvester. The candidate worked closely with the partner company for the deployment of the system and integration with relevant infrastructures of both hardware and software. The candidate supported the Senior Researcher in the improvement of the paper in response to the review comments. The candidate is the corresponding author and as a result, an active reviewer of papers related to the topic.

Publication 4: Soft robotic caterpillar with wirelessly powered shape memory alloy actuators

The candidate was the lead author, producing the majority of the text and coordinating the input of the contributing authors, and supported the second author in the finalisation and submission of the draft. The candidate created the concept for the wireless power transfer to multiple shape memory actuators. The candidate was responsible for the design, implementation and evaluation of the caterpillar receiver coils and their combination with the shape memory alloy actuators. In collaboration with the designer, the candidate implemented and characterised the transmitter coil and directly produced the related results. The candidate was responsible for the integration of the wireless power transfer system with the robotic caterpillar, the implementation of the working system, and all results presented in the paper. The candidate is the corresponding author.

Publication 5: Development and evaluation of a reader device for fully passive wireless sensors

The candidate was the lead author responsible for the drafting and submission of the paper. Under the supervision of the Senior Researcher, the candidate was the main technical contributor responsible for the design, implementation and characterisation of the integrated reader device which forms the core contribution of the work. The candidate was responsible for the characterisation tests of the reader itself, and the sensor range readout tests comparing the two sweeping techniques. The candidate integrated the theory, and frequency sweeping sections, and results on the evaluation of the resolution enhanced reader in cooperation with the contributing authors. The candidate is the corresponding author.

Publication 6: RF Communications for a swallowable capsule

The candidate was the lead author responsible for the drafting and submission of the paper, and its improvement based on reviewer comments. The candidate cooperated with contributing authors in the design and implementation of the radio communication and was responsible for its integration with the capsule. The candidate performed the evaluation of the radio system and generated all the results of the paper. The candidate gave the oral presentation of the paper and is the corresponding author. 
Publication 7: Development and evaluation of an on-chip potentiostat for biomedical applications

The candidate was the lead author responsible for the drafting and submission of the paper, and its improvement based on reviewer comments. The candidate collaborated with the IC designer in the design of the integrated potentiostat and was fully responsible for the characterisation results. The candidate gave the oral presentation of the paper and is the corresponding author.

Publication 8: Activation and identification of fully passive wireless sensors

The candidate was the lead author responsible for the drafting and submission of the paper, and its improvement based on reviewer comments. The candidate created the concept for activation and identification of the sensors and generated the evaluation results presented in the paper. The candidate presented the poster of the paper at the conference and is the corresponding author.

Publication 9: Development of a reader device for fully passive wireless sensors

The candidate was the lead author responsible for the drafting and submission of the paper, and its improvement based on reviewer comments. Under the supervision of the Senior Researcher, the candidate was the main technical contributor responsible for the design, implementation and characterisation of the integrated reader device which forms the core contribution of the work. The candidate was responsible for the characterisation tests of the reader, and the sensor readout tests. The candidate presented the poster of the paper at the conference and is the corresponding author. 



\section{Introduction}

\subsection{Research Problem}

Sensors are used increasingly in a wide range of applications for gathering information about an environment or process. There are many applications which would greatly benefit from sensing elements, but sensors are not currently feasible due to the requirement of an electrical energy source or data connection of some kind. Therefore, to enable widespread deployment of sensor systems in our world, they must be wireless and power autonomous. There is no universal solution to achieve such a device. Instead, a holistic and system level approach must be taken that considers the sensor, its readout and control circuitry, wireless communications and powering methods available in the context of the application requirements and sensor system lifetime.

Sensors can be interfaced with a wired connection which allows direct powering and a robust communication interface. However, wired connections are costly, subject to wear and tear, and impractical or even impossible in some applications where the sensor has to move or is fully encapsulated. Therefore, wireless communication is highlighted as a key requirement to enable the wide adoption of Internet of Things (IoT) sensing devices. The wireless communication system, when active, often consumes an amount of energy an order of magnitude greater than that required for sensor read out, signal conditioning and data processing. While short communication bursts can bring the average consumption of radio devices to a more tolerable level, it is clear that intense attention must be paid to the wireless communication scheme in order to evolve the system towards power autonomy.

A sensing system with electrical readout and wireless communication relies on the availability of an energy source of some kind. Accepting that fact, an energy autonomous sensor can be defined as "an electronic system (sensor) that has been designed to operate and/or communicate as long as possible in known/unknown environments providing, elaborating and storing information without being connected to a power grid" [1]. In the context of this thesis, a sensor is considered energy autonomous if it can operate as required, throughout its measurement lifetime without the need for energy related intervention by the user.

The research problem of this thesis is the investigation and demonstration of the feasibility of power autonomous sensors in a wide range of applications. Achieving power autonomy requires a two front approach; addressing both the need and the supply. The optimisation of power consumption requirement of 
the sensor is considered a necessity, with special attention to the consumption in sleep modes for sensors measuring at significant time intervals. As stated above, and as subsequent sections of this thesis will demonstrate, the radio communication infrastructure represents a critically important challenge in the power optimisation, which itself can also be exploited as a power delivery mechanism. The systems developed in the work allow the exploitation of energy sources appropriate for the application. The developed prototypes include battery power in the case of short lifetime devices, energy harvesting where reliable sources exist, wireless power transfer, and radio backscattering techniques.

\subsection{Motivation}

Digitalisation is changing the world as we know it, at a rate faster than we have ever seen. Already, whole industries have been created and destroyed through the penetration of digital devices and services. The information age has already progressed to the stage of big data and artificial intelligence. Thus far, the digital revolution has been driven largely from the evolution and wide scale penetration of complex devices such as personal computers and smartphones. In parallel, their distributed computational power and seamless connectivity contributes towards current and future digitalisation trends. In recent years, smaller, simpler and cheaper connected devices are evolving to the point of wide adoption. As such, the lower level distribution of intelligence and connectivity gives rise to the emerging IoT, here proposed to be the key driver in the next wave of evolution in the age of digitalisation.

An IoT device can be defined as a stand-alone, identifiable measurement or actuation device that can be connected to the internet. The extent of IoT devices is illustrated in Figure 1, reproduced from ${ }^{[2]}$. The nearest range devices in the Personal Area Network (PAN) include wearable and person peripheral devices, remote consumer electronics, short-range safety and control devices, automotive devices, robotics and toys. These kinds of devices traditionally connect to the internet via short range radios (max few 10s of meters) such as Bluetooth, through a gateway device, like smartphone or personal computer. At the next range, Local Area Network (LAN) devices achieve a range in the high $10 \mathrm{O}-100$ $\mathrm{m}$ using $\mathrm{Wi}-\mathrm{Fi}$, or in some cases lower power radios such as Bluetooth. This category of device largely applies to building level and includes connected instruments, healthcare devices, entertainment devices, domestic appliances and building automation for domestic, commercial and industrial applications. A category of IoT devices is emerging in the Wide Area Network (WAN) scale. These exploit evolving long range radio technologies such as Low-Power LongRange (LoRa) and Narrowband Internet of Things (NB-IoT) to achieve readout ranges of $100 \mathrm{~s}$ to $10 \mathrm{sm}$. These devices include automotive navigation devices, agricultural and environmental sensors, industrial sensors, security devices and infrastructural monitoring. The work of this thesis focuses on shortrange devices that fall under the categories of PAN and LAN. 


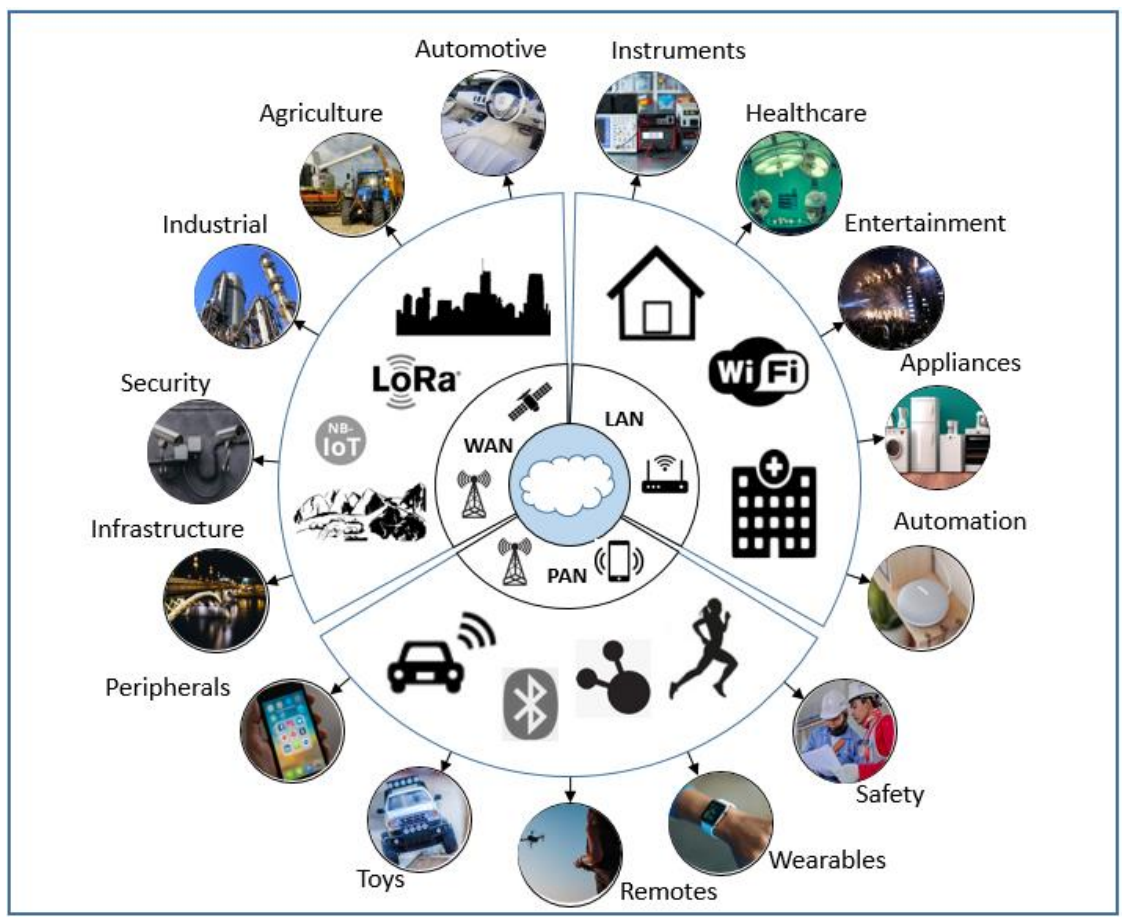

Figure 1. IoT devices and applications, subdivided by network reach (PAN, LAN and WAN). Reproduced from ${ }^{[2]}$ using stock images.

The potential of IoT devices is far-reaching and it can be expected to impact almost every industry. A 2018 Statista dossier on IoT ${ }^{[3]}$ reports the exponential growth of annual global generated revenue from $\$ 33$ billion in 2012 to $\$ 94$ billion in 2017. The key segments that drive IoT are manufacturing, construction, retail, utilities, healthcare, automotive and health sciences. There are thousands of companies driving the IoT revolution by producing both devices and IoT infrastructures. The report ${ }^{[3]}$ predicts that almost $80 \%$ of the global IoT revenue in 2017 was shared among 2 sub-sectors; Connected Cities (41\%) and Industrial Internet (38\%). The remaining share is predicted to be divided among Wearable Systems (13\%), Connected Vehicles (5\%) and Connected Homes (3\%). Stasticia predicts the number of IoT devices installed to grow exponentially from 20 billion in 2015 to 75 billion in 2025. The revenue and predicted growth of the global IoT market is illustrated in Figure 2.

The size of the IoT market worldwide was estimated to be valued just under $\$ 157$ billion in 2016, and is projected to grow to $\$ 457$ billion in 2020 [3]. Some of the companies at the forefront include Google, Apple, Microsoft, Amazon, IBM, Cisco, Verizon and GE. Many of these companies have developed IoT platform infrastructures (Amazon Web Services, Microsoft Azure, and Cisco Connect) in addition to devices. 


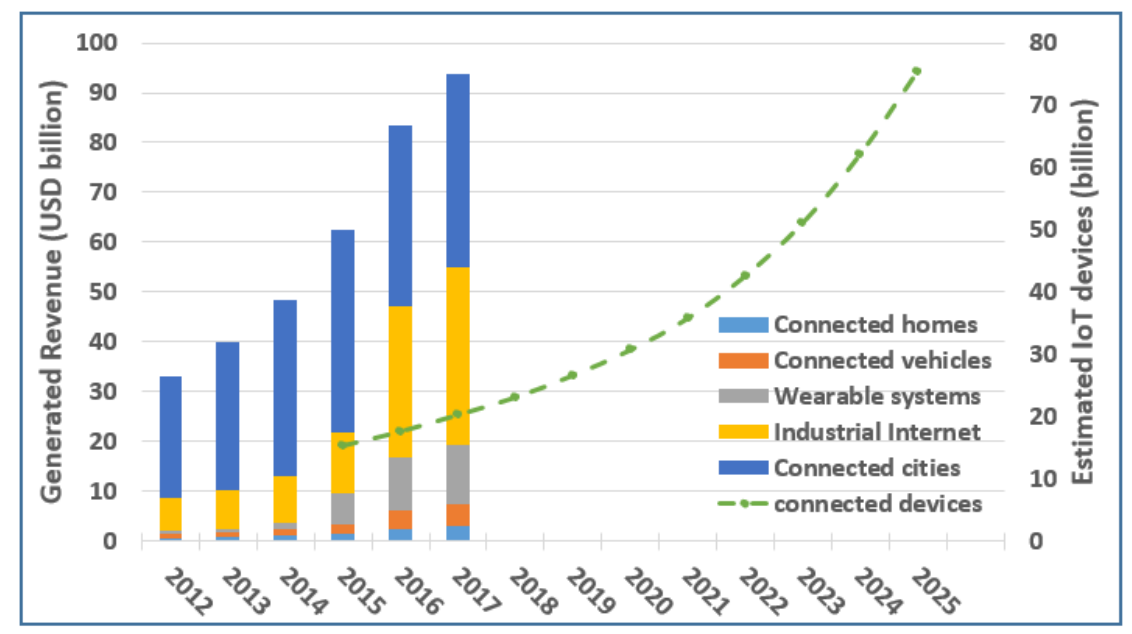

Figure 2. Global generated revenue in loT devices and applications, divided by sub-sector from 2012-2017 and the projected exponential growth of loT devices from 2015-2025, reproduced from ${ }^{[3]}$.

As the number of IoT devices grows exponentially, so too will the challenges associated with their adoption. The factors which hinder the adoption of IoT devices are cost, size of the connected device, power, communication and ease of deployment. It is clear that the value provided by an IoT device must exceed the cost of its production, cloud based service, installation and maintenance. In the majority of cases the power source can be the physically largest and most expensive component of the system. In the case of battery powered devices, as applies to the majority in use today, the maintenance associated with the replacement of drained batteries can create a cost burden that can exceed the value produced by the device. Furthermore, battery technologies are made from scarce, and in many cases, toxic materials (such as lithium), which create difficult to handle waste. Considering the scale of IoT devices, in the tens of billions, it is clear that the battery powered solution equates to an environmental catastrophe.

There is a clear and present need for the development of new and innovative IoT device powering schemes which can alleviate the effects of battery powered devices. The impact of removing batteries from IoT devices includes reduced size and cost, elimination of battery replacement, maintenance and reduction of difficult to process toxic waste. 


\subsection{Scientific Contribution}

The scientific contribution of this thesis includes the development of four novel and mutually exclusive systems. While each has their unique contributions to the state of the art, summarised in this section, they follow an evolution from a traditional battery powered system towards power autonomy. As the development evolves beyond battery power, the systems exploit energy harvesting, wireless power transfer and backscattering sensors based on the third order intermodulation principle.

The first of the developed systems relates to a wireless swallowable capsule for gastrointestinal disease diagnosis. Publication I includes a detailed review of the state of the art, both commercially and in research, in swallowable capsule technology. At the time of publication of this thesis, this article remains one of the most cited papers on the topic, with 75 citations as of $1^{\text {st }}$ December 2018. The capsule developed in this work, described in Publication II, represents the world's first swallowable device with an electronic tongue sensor and potentiostatic readout technique. In addition to the system level description in Publication II, this work includes two conference papers; Publication VI describing the development of a wireless communication system for the swallowable device and Publication VII on the development of an integrated potentiostat circuit for readout of the electronic tongue sensor. The developed device enhances the possibility of in-vivo diagnosis of gastrointestinal diseases such as ulcerative colitis and Crohn's disease. Furthermore, the device is currently being exploited in other applications such as the monitoring of reaction processes inside a closed bioreactor system.

The second system developed in this work relates to energy autonomous valve leakage detection for petrochemical valves, as described in Publication III and live-demonstrated to peers in the field in [4]. A novel MEMS acoustic emission sensor is applied to detect minute vibrations caused by the turbulent flow of high-pressure fluid as it encounters micro fractures in the valve. The system is developed around a novel ultra-low-power wake-up topology based on passive down conversion mixing. The wake-up radio allows the sensor node to be fully deactivated, and triggered via a low power wake-up IC. Power autonomy is provided using a thermal energy harvester in the sensor node and an industrial current loop energy harvester in the base station device. The developed system has the potential to detect valve micro fractures and highlight valve deterioration long before a catastrophic valve rupture. The sensor is shown to achieve improved sensitivity when compared to a commercial sensor and the wake-up radio is compared with the state of the art in Publication III.

The third system developed in this work relates to a wirelessly powered soft robotic caterpillar, described in Publication IV. The system utilises resonant inductive coupling to two receiver coils combined with shape memory alloy actuators. Inductively coupled current in the receiver coil is converted to heat in the shape memory alloy which causes contraction needed to provide locomotion for the robot. A novel system architecture is presented for the control of multiple receiver actuators by controlling their resonance frequency and sweeping the 
stimulation signal to a wideband transmitter loop. To the authors' knowledge, at the time of publishing, this was the world's first wirelessly powered soft robot.

The final system developed in this work relates to fully passive wireless sensing utilising passive backscattering and the third order intermodulation principle. In particular, this thesis focuses on the design, implementation and test of an integrated reader device for interrogating passive wireless sensors, introduced in Publication IX and described in detail in Publication V. The work focuses on the enhancement of the readout range of the device, and the improvement of the readout resolution. In addition, the work of this thesis demonstrates a method of integrating standard Radio Frequency Identification (RFID) technology with the fully passive sensors for activation and identification, described in Publication VIII. The contributions of this work are considered key steps towards the practicality of the wide deployment of the zero power sensor system. 


\section{Background and State of the Art}

\subsection{Wireless Sensor System Architecture}

A typical topology of a wireless sensor network appropriate for use in IoT applications is illustrated in Figure 3a. The network comprises of a number of remote sensor nodes deployed over an area of interest connected wirelessly to a gateway receiver. Each sensor may communicate directly with the gateway in a 'star' configuration as shown in Figure 3a, or connections may be routed between them in a multi-hop nature. Depending on the range and extent of the network, standard radio technologies used may include Bluetooth, ANT, RFID etc. (short range), Wi-Fi etc. (medium range) or LoRa, NB-IoT and 3/4/5G (long range). The gateway node shown in Figure 3a may be fixed to a mains power source and hardwire internet connected, or it too may be off grid and wirelessly connected to another gateway higher in the network hierarchy. The gateway relays sensor data from the network, increasingly to cloud based servers and services where it can be interfaced to remote users through a multitude of interface devices like computers, smartphones etc.

A typical hardware architecture of a single wireless sensor - gateway pair is presented in Figure 3b. The device consists of a sensor element extracting some information as required by the application. In general, the sensor element converts some physical parameter to an electrical signal. The sensor is controlled and read out by some electronic front end circuitry, which provides a conditioned signal for digitisation in an Analogue to Digital Converter (ADC). The microcontroller (MCU), typically including the $\mathrm{ADC}$, comprises the 'brain' of the system managing all processes in the device. Memory is often included, either as part of the MCU or an external component. A radio transceiver receives data from the MCU and converts it to a modulated Radio Frequency (RF) carrier for transmission. The antenna, often protruding from the device, is a key element in coupling the modulated RF carrier to electromagnetic waves for transmission through the air. The power supply for the remote sensor is predominantly based on a battery, but can also be some kind of energy harvesting device. On the gateway side, the electromagnetic wave transmission is coupled from the air to an electrical signal by the antenna, and demodulated in the radio transceiver. The MCU receives and processes the data for storage in memory or real time communication of the data. The data can be directly interfaced to the end user, but increasingly gateways are internet connected to the cloud where authorised remote users can access the data anywhere at any time. The power supply for the 
gateway is often provided by mains power, but some applications require that it too is battery powered.

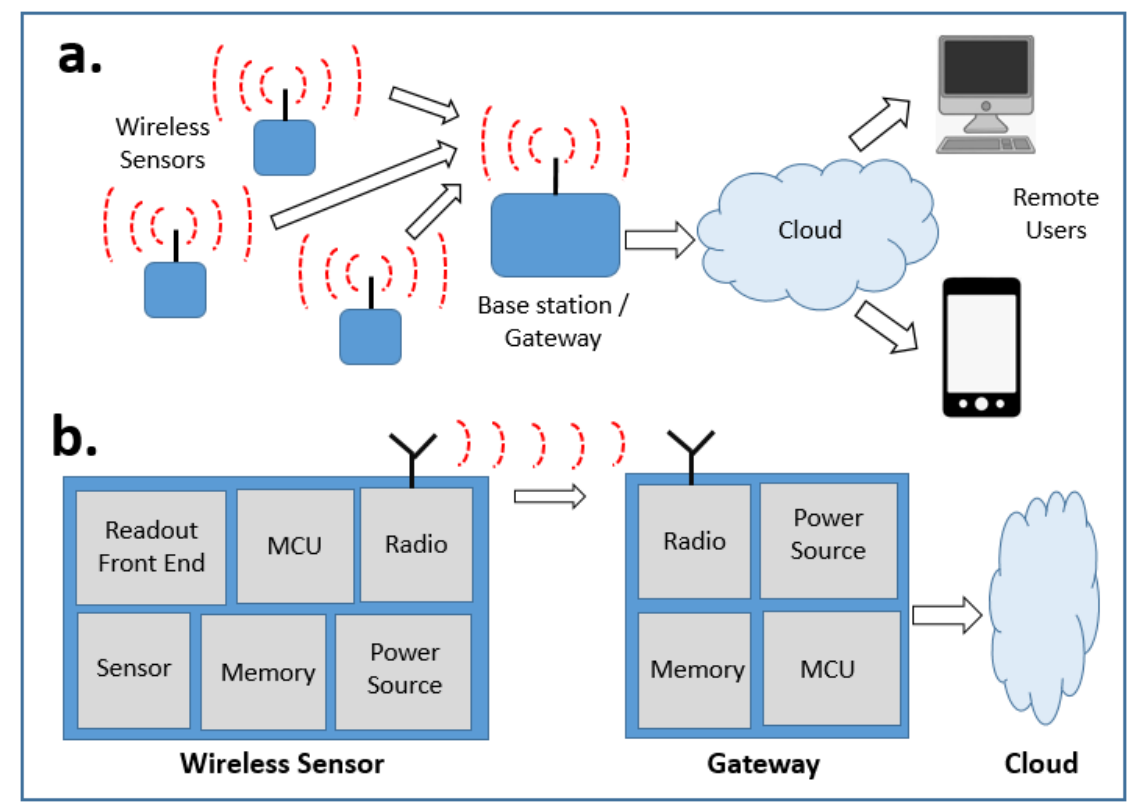

Figure 3. Anatomy of a wireless sensor network and devices. (a) Typical wireless sensor network architecture, in star unidirectional communication configuration. (b) Architecture of a single wireless sensor and gateway device.

\subsection{Power Consumption Overview}

A key consideration in the deployment of wireless sensor networks, and core to the scope of this doctoral thesis is the power requirement and supply. This is highlighted as the most critical aspect of the sensor nodes themselves, where a deploy-and-forget paradigm is most desirable. Examining the power requirements of each component of the sensor node, let us first consider the sensor itself and its readout circuitry. The sensing element itself may be passive, whereby the physical measurement is directly manifested as an electrical signal without power input, however most often some bias or stimulation energy is needed. The readout electronics often consist of an analogue front end circuit including biasing, stimulation and signal acquisition, including amplification and filtering, and typically consumes some 1s - 10s mW. Most often in IoT applications the sensor measurements can be taken periodically at long intervals so that the sensor and readout circuitry power consumption contribution depends dramatically on the measurement interval.

The microprocessor digital core of the sensor node typically consumes a significant proportion of the system power when in the active state in the range of 10 s of $\mathrm{mW}$, but it can often spend the vast majority of the time in a low power sleep state in between measurements consuming single digit uW. Where 
memory is included in the sensor node, it typically comes either as a RAM type memory which is written with some $\mathrm{uW}$ consumption but requires a constant nW range energy supply to retain data, or as a flash type memory which needs some 10 s of $\mathrm{mW}$ programming energy but can retain data without constant energy supply.

As the power optimisation of sensors, readout electronics and microcontrollers advances the radio communication components become increasingly significant in the power consumption profile of the sensor node. The active consumption of radio components falls typically in the range of $10 \mathrm{sW}$ for short range radios like Bluetooth Low Energy (BLE), increasing to around $100 \mathrm{~mW}$ for long range networks like LoRa and NB-IoT and around $500 \mathrm{~mW}$ for cellular radios like Long Term Evolution (LTE). Like the sensor and readout electronics, the radio components are duty cycled so that their overall consumption depends highly on the communication intervals.

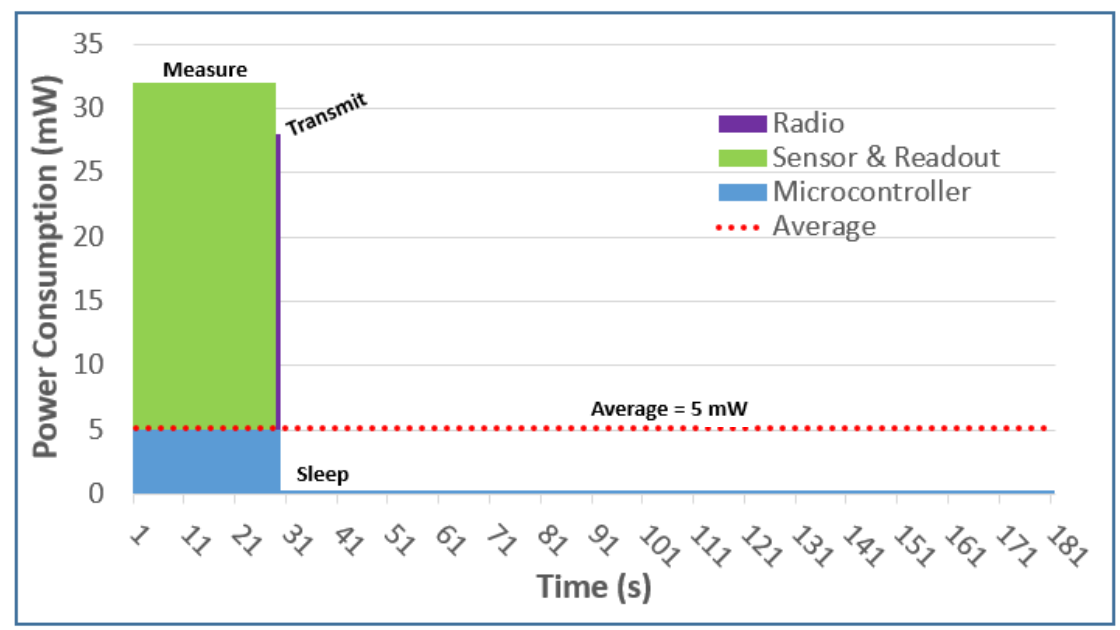

Figure 4. Typical power consumption profile of a wireless sensor node, using the device of Publication II as an example. In this illustrative example the sensor is measured for $26 \mathrm{~s}$, radio transmits for $1 \mathrm{~s}$ and the node is in sleep mode for $120 \mathrm{~s}$.

The typical power consumption profile of a wireless sensor node is illustrated in Figure 4. Here the power consumption values measured in the wireless swallowable capsule of Publication II are used as an example. During the measurement cycle, lasting $26 \mathrm{~s}$, the microcontroller accounts for $5 \mathrm{~mW}$ power consumption and the sensor readout electronics $27 \mathrm{~mW}$. During the communication cycle, lasting $1 \mathrm{~s}$, the microcontroller still consumes $5 \mathrm{~mW}$ and the radio device consumes $23 \mathrm{~mW}$. Given the sleeping time of $120 \mathrm{~s}$, the average power consumption of the device is $5 \mathrm{~mW}$. Considering a longer sleeping time of $1 \mathrm{hr}$, the average consumption can be calculated to be approximately $600 \mathrm{uW}$. It should be noted here that the electrochemical measurement technique used in the work of Publication II is slow and more power hungry when compared to a great proportion of the measurements in IoT applications. One example with simple temperature sensing and BLE radio achieves an average consumption of $51 \mathrm{uW}$ [5]. 
In the context of this thesis, a sensor is considered energy autonomous if it can operate as required, throughout its life cycle without the need for energy related intervention by the user, following from the definition of power autonomy in Section 1.1. It follows directly, that all wired and mains powered sensors satisfy the definition of power autonomy. However, as highlighted in Section 1.1, the sensors relevant for mass deployment in IoT applications need also to communicate wirelessly. To achieve energy autonomy in a wireless sensor network there are three possibilities: (i) to store sufficient energy for the lifetime of the device, (ii) to harvest ambient energy in the device environment and (iii) to wirelessly transmit the energy. The state of the art in terms of (i) is detailed in Section 2.3.3, (ii) is explored in Section 2.3.4, while Sections 2.3.5 and 2.3.6 are dedicated to (iii).

\subsection{State of the Art}

\subsubsection{Power Consumption Optimisation of Wireless Sensors}

The power optimisation of wireless sensors must be considered on the device level, and also on the systems level for the whole Wireless Sensor Network (WSN). In recent years, there has been a trend towards power consumption reduction in electronic circuits, relevant for use in wireless sensor systems. For example, DSPs have reached to $10 \mathrm{uW} / \mathrm{MMAC}$ (millions of multiples and accumulations per second), the ADCs figure of merit is now $15 \mathrm{fJ} /$ conversion step, electrical power conditioning devices are reaching near unity efficiency, and radios are achieving less than $1 \mathrm{~nJ}$ per communicated bit ${ }^{[1]}$. The progress in power optimisation of the electronic components of wireless sensor systems is an essential prerequisite for the achievement of a power autonomous sensor system. Based on this optimization, numerous power techniques become feasible as energy supplies for wireless sensor systems, therefore opening up the possibilities for power autonomy. The power optimization of the sensor systems in this work is determined as per the application requirements of each system, and available energy sources. This ranges from low need of optimisation in the short lifetime device of Publication II, to the intense need of sleep state power reduction in the device of Publication III, and further intensity of the low power operation of the fully passive sensor in Publication V.

While network level optimisation is considered outside the scope of this thesis, some comments on the state of the art in that area are included for completion. On the network level, the development of power optimized communication protocols for wireless sensor networks is an intense area of research. An effective generalised power calculation model presented in the literature, provides a framework for the estimation of energy life cycles of devices in a network ${ }^{[6]}$. One survey of energy saving techniques provides a comprehensive set of energy optimisation techniques depending on the network architecture, scale and wire- 
less protocol [7]. There are a host of strategies for power optimisation in traditional WSNs, evaluated in the literature ${ }^{[8]}$. These include smart resource allocation, opportunistic transmission / scheduling, adaptive routing / clustering, mobile relays and sinks, optimal deployment, network coding and data correlation, in addition to hardware oriented techniques such as energy harvesting and beamforming. Time Division Multiple Access (TDMA) is one simple and energy efficient protocol for low data rate WSNs. In this protocol, sensor nodes activities are scheduled for the optimal energy consumption. One significant work in this area presents generalised TDMA algorithms for both homogenous and heterogeneous WSNs [9].

\subsubsection{Ultra-Low Power Wireless Communications}

The state of the art in commercially available short range ultra-low power radios includes BLE, ZigBee and ANT and their use is widespread in the IoT ecosystem including; consumer electronics, wearables, sports and fitness, building automation and wireless sensor networks. Detailed comparison of these standards in terms of their power consumption highlights BLE as the lowest power solution ${ }^{[10]}$. With reference to industry leading BLE chips from vendors CYPRESS ${ }^{[11]}$ and Texas Instruments ${ }^{[12]}$, active consumptions of 15-20 mA are typical, combined with sleeping currents in the order of $100 \mathrm{nA}$ - 1s uA give average consumptions of $17 \mathrm{uA}^{[13]}$ and $24 \mathrm{uA}{ }^{[14]}$ for a $1 \mathrm{~s}$ connection interval and zero data payload. More recently, the Nordic Semiconductor wireless MCU (nRF52) leads the field with active consumptions as low as $5.2 \mathrm{~mA}$ while transmitting at $\mathrm{o} \mathrm{dBm}$, and receiver active consumption as low as $5.4 \mathrm{~mA}$ [15].

The average consumptions of BLE are quoted with the assumption of perfect synchronisation between devices, which is feasible only for short connection intervals, on the order of $100 \mathrm{~s}$ ms. As connection interval increases, accuracy of synchronisation decreases. This increases the probability of latency in the receiver waiting for a target response, manifested as wasted energy on the receiver side. Therefore, BLE or other duty cycled communication techniques are not ideally suited to cases where energy consumption is critical on both sensor nodes, and interrogation gateways. Moreover, applications where the measurement interval is very long, on the order of minutes or hours to weeks or more, have an overall power consumption which is overwhelmingly dominated by consumption in the sleeping state. As a result, duty cycled radio standards waste a significant amount of power in unnecessary connections. Applications including building automation, environmental monitoring, and industrial condition monitoring can benefit from a fully asynchronous wake-up topology where sensor nodes can be activated at any time from a base station. With such a profound need, wake-up radio technologies is an area of intense research and numerous topologies are found in the literature ${ }^{[16]}$. A majority of the topologies presented there remain at the conceptual or prototyping stage. We consider only those circuits that are readily available for exploitation.

Considering the CYPRESS commercially available BLE chip ${ }^{[11]}$ from the perspective of a wake-up radio, the device gives a sensitivity of $-89 \mathrm{dBm}$, with an 
average consumption of $27 \mathrm{uA}$ at $1.8 \mathrm{~V}$. However, a latency of $1000 \mathrm{~ms}$ can cause especially high consumption in the interrogator device. In addition to this work, a number of additional discrete, commercial off-the-shelf (COTS) diode detector asynchronous correlator devices are presented in the literature operating at different Industrial, Scientific and Medical (ISM) frequency bands. One such device ${ }^{[17]}$, operating at $869 \mathrm{MHz}$ gives a sensitivity of $-52 \mathrm{dBm}$ with an average current consumption of $2.8 \mathrm{uA}$ at $3 \mathrm{~V}$ and a latency of $13 \mathrm{us}$. Another device [18], operating at $434 \mathrm{MHz}$, averages consumptions of just $280 \mathrm{nA}$ at $1.5 \mathrm{~V}$. This is achieved using a complex diode detector circuit and digital logic to wake a microcontroller via the SPI port. In addition, a semi-passive MHz RFID based device can be applied as a wake-up generator ${ }^{[19]}$ achieving $-27 \mathrm{dBm}$ of sensitivity, a consumption of $900 \mathrm{nA}$ at $3 \mathrm{~V}$ and a latency of $1 \mathrm{~ms}$.

The key background technology in this work, as used in Publication III, is the wake-up radio based on a discrete COTS diode detector asynchronous correlator [20]. The topology operates at $2.45 \mathrm{GHz}$ and achieves a sensitivity of $-50 \mathrm{dBm}$, with a sleeping consumption of $6.8 \mathrm{uA}$ at $3 \mathrm{~V}$ and a latency of $13 \mathrm{~ms}$. It is the core technology that enables asynchronous wake-up and interrogation of sensor nodes, allowing a minimal optimised power consumption in both the measurement nodes and master gateway.

\subsubsection{Energy Storage for IoT Sensors}

In terms of energy storage, options include fuel cells which store and convert energy from chemical sources, batteries (primary and secondary) which store energy in electrochemical form and super capacitors which store energy with charge. Fuel cells provide the highest energy densities in order of $10^{5} \mathrm{~J} / \mathrm{kg}$ but with very slow conversion rates so have poor power capabilities $(10-100 \mathrm{~W} / \mathrm{kg})$. Batteries lie in the middle range of both energy density $(1,000-10,000 \mathrm{~J} / \mathrm{kg})$ and power capability (100 - 1,00o W/kg). Capacitors, while poor in terms of energy capacity $(10-100 \mathrm{~J} / \mathrm{kg})$, are capable of providing huge instantaneous power (up to $10,000 \mathrm{~W} / \mathrm{kg}$ ) [21].

The vast majority of wireless sensors in IoT applications are powered with primary batteries. A review of batteries for IoT devices ${ }^{[22]}$ highlights the consideration of application requirements, in particular lifetime, in the selection. The main battery technologies highlighted includes primary zinc manganese dioxide, lithium and zinc air, and secondary (rechargeable) lithium ion and nickel metal hydride cells. In the early days AA and AAA sized alkaline cells dominated with approximate power densities nearing 2400 and $1000 \mathrm{mAh}$ respectively, each at 1.5 V. As devices have become smaller and more power efficient primary lithium-ion cells have become popular, selected for their high specific energy, high efficiency and long life. One of the most prevalent devices, the CR2032, provides $210 \mathrm{mAh}$ which is enough to achieve a four year lifetime with the aforementioned temperature sensor device [5], or an estimated 9.2 days for the sensor systems consumption shown in Figure 4. Lithium-ion battery technology is the 
leading technology for powering IoT. While research efforts are intense, the advancement of lithium battery has been incremental, falling behind the accelerating scale and needs of IoT device ecosystem [23]. The environmental impact of the toxic components of single use primary lithium-ion cells is clear. Numerous technologies for recycling them exist ${ }^{[24]}$. However since the costs outweigh the recoverable value, the vast majority of lithium-ion batteries end up in landfill.

The device presented in Publication II applies a lithium manganese dioxide primary cell, while the device of Publication III applies a secondary rechargeable lithium polymer cell in combination with the thermal energy harvester.

\subsubsection{Energy Harvesting Sensor Systems}

Energy harvesting wireless sensor systems form the largest category of power autonomous sensors. The most widely reported sources in the literature include solar, motion/vibration, thermal and electromagnetic ${ }^{[25]}$. These are reported to have been harvested to supply $10 \mathrm{uW}-10 \mathrm{~mW} / \mathrm{cm}^{2}$, with respective harvested power densities per square centimetre are reported as follows: solar - $10 \mathrm{u} / 10$ $\mathrm{mW}$ (indoor/outdoor), mechanical - 4/100 uW (human/industrial), thermal $30 \mathrm{u} / 1-10 \mathrm{~mW}$ (human/industrial) and RF - $0.1 \mathrm{uW}{ }^{[26]}$. All energy harvesting systems face challenges relating to efficiency over an unpredictable range of source energy availability. Wireless sensor networks especially suffer intense challenges of optimisation of resource allocation under the limitations implied by a scarce and unpredictable harvested power source ${ }^{[27]}$. Open research questions on energy harvesting sensors relate to miniaturisation, multisource harvesting, operational adaption to available energy, energy availability prediction, efficiency, reliability and effective energy storage ${ }^{[28]}$.

Solar energy harvesting relies on the direct conversion of light to electrical energy in a photovoltaic cell [29]. A core challenge in solar energy harvesting relates to adaptation of the harvester circuit for the extraction of maximum available power. No less than 19 methods of 'maximum power point tracking' are reported in the literature [30], many of which are widely applicable to energy harvesting in general. To enable solar powered sensor systems, small and highly efficient cells and harvester circuits are required, in addition to the ability to store energy and adapt to fluctuations in light intensity ${ }^{[31]}$. Solar powered sensor platforms include energy storage and can be found with both rechargeable batteries [31] and super capacitors [32]. The advantages of solar include reliability of supply and low maintenance cost. Disadvantages relate to the relatively large area needed and challenges with efficient storage of harvested energy in leaking batteries and self-discharging super capacitors.

Thermal energy harvesters exploit the thermoelectric effect where the flow of charge carriers between hot and cold regions generates a current [33]. Thermally powered sensor systems have been emerging for industrial and, more recently, wearable [34] applications. A thermal energy harvester has been reported in [35], exploiting heat at domestic radiators and powering a ZigBee wireless sensor node. Thermal energy harvesting remains an area of research interest with ad- 
vances in microsystem integration driving more effective thermoelectric elements and more efficient converter circuits [36]. The advantages of thermoelectric energy harvesting are low maintenance costs, stable energy with stable temperature difference, and no short circuit dangers on Thermoelectric Generator (TEG) elements. Disadvantages relate to the uncertainty of the heat source, low energy conversion efficiency and the inefficiency of storing harvested power.

Mechanical energy harvesting converts movement or vibrational energy to electrical energy by exploiting the piezoelectric effect, the electromagnetic effect or the movement of a charged mass. Mechanical energy has been effectively harvested from both human and industrial sources [37]. Motion based energy harvesters are being applied to enable wearable batteryless sensors [38]. The key disadvantage with mechanical energy harvesters is that the device's region operation is often in a narrow range of motion and acceleration. Therefore these devices must be tuned carefully to the application and suffer poor efficiencies as the force or motion goes beyond the tuned operating point. Further, due to the presence of moving elements, motional energy harvesters can create costs relating to maintenance.

RF energy harvesting extracts energy from radio signals, originating from ubiquitous radio transmitters, in the frequency range from $3 \mathrm{kHz}-300 \mathrm{GHz}$ fields. In this work, RF energy harvesting is highlighted as exploiting only ambient signals, as opposed to RF power transfer from dedicated sources. RF energy harvesting systems are reported in the literature operating from $450 \mathrm{MHz}$ - $2.45 \mathrm{GHz}{ }^{[39]}$, and in some cases up to $5.8 \mathrm{GHz}$ and beyond [40]. The harvesting system typically comprises of an antenna and matching network, coupled with an RF to DC converter and voltage multiplier and an energy storage device in the form of capacitor or battery, well described in ${ }^{[39,40]}$. The device can also be manifested as a rectenna, a device which combines the antenna and the RF to DC rectifier. The optimisation of RF energy harvesting circuits and evaluation of their suitability to power embedded sensor systems are areas of research interest. The literature shows ${ }^{[40]}$ a wide range of efficiencies from $90.6 \%$ at 2.4 $\mathrm{GHz}$ and input power of $39 \mathrm{dBm}$ down to $1.2 \%$ at $900 \mathrm{MHz}$ and $-14 \mathrm{dBm}$. A pattern is observed from the collection of work in ${ }^{[40]}$, where lower frequency 900 MHz and 2.4 GHz RF energy harvesters are feasible for 10-20\% efficiencies from $-15 \mathrm{dBm}$ to $-22 \mathrm{dBm}$ input powers. Applications for RF energy harvesters are wide and include WSNs [41]. The advantage of ambient RF energy harvesting is that it exploits otherwise wasted energy, however the disadvantages are many. In the literature, only high input powers are evaluated which raises doubt as to the applicability in realistic ambient RF power levels.

The measurement system presented in Publication III of this work applies a thermal energy harvester in the sensor node, and an industrial current loop harvester is the gateway to give a fully power autonomous system. 


\subsubsection{Wireless Power Transfer}

Wireless power transfer is differentiated from RF energy harvesting in that a dedicated device transmits the power to the node. In keeping with the scope of this thesis, inductively coupled wireless power transfer will be here considered, and far field backscattering methods are addressed in Section 2.3.6. The technique of wireless power transfer was demonstrated by the renowned Nicola Tesla in the early $18^{\text {th }}$ century ${ }^{[42]}$. Although Tesla's dream of free universal wireless power for all has not quite been realized, there has been a reawakening of the topic since the 2007 MIT demonstration of the wireless transmission of 60 $\mathrm{W}$ of energy, a distance of $2 \mathrm{~m}$ at $40 \%$ efficiency using resonant inductive coupling ${ }^{[43]}$. Since then, there has been a renewed interest in power without wires ${ }^{[44]}$, significant progress in mid-range wireless power transfer [45], and key developments in the combination of data and power transfer ${ }^{[46]}$. Numerous specific wireless power transfer systems have emerged for applications in automotive [47], consumer electronics [48] and charging of implanted devices [49]. A thorough analysis of wireless power transfer, its history, techniques, standards and applications is provided in the literature ${ }^{[50]}$.

Commercial realisations of wireless power transfer have, until recently, remained limited to a number of traditional applications. The most recognisable of these employ non-resonant inductive coupling and include wireless recharging of electric toothbrushes and razors, and induction heating stovetops. Within the last two decades, resonant inductively coupled near field RFID has found numerous applications in access control and ticketing systems, and is a basis for Near Field Communication (NFC) ${ }^{[51]}$. In 2008, the Wireless Power Consortium released the Qi standard ${ }^{\left[5^{2}\right]}$ to standardise wireless power transfer of low powers $(<5 \mathrm{~W})$ over short distances $(5-40 \mathrm{~mm})$ using resonant inductive coupling in the region of $100-200 \mathrm{kHz}$. The first significant commercial device with Qi enabled wireless charging was the Nokia Lumia 930, and to date it has been included in smartphones of almost all major vendors and additionally in tablets, power banks, speakers, cameras, smartwatches and other computer peripherals. The same MIT development team that reinvigorated the topic in 2007 generated a spin-off company WiTricity. They now claim to be ready to enter the electric vehicle charging market in 2018 with 90-93\% efficient transfer of 3.6-11 kW [43].

Wireless power transfer to multiple receivers, and in particular, robot swarms, is an area of research interest in the last decade. A number of robotic devices are described in the literature with low frequency Wireless Power Transfer (WPT) and relatively large receiver coils. Many of these are wheeled robots [53], though some manifestations also include ambulatory 'insect' type locomotives [54]. WPT to Shape Memory Alloy (SMA) actuators has been successfully implemented on a micro scale [55]. This work enabled the addressed actuation of microfluidic channels using a frequency multiplexing technique. Frequency multiplexing is also applied for wireless actuation to multiple joint origami structures in a manner similar to this work ${ }^{[56]}$. A wireless earthworm-like device with SMA actuators is reported in the literature ${ }^{[57]}$. However, while the device is controlled wirelessly, the large currents for the SMA actuators are provided by a battery. 
As a direct prelude to this work, a caterpillar-like soft robot has been developed [58]. The robot locomotion is provided using SMA actuators stimulated with Pulse Width Modulated (PWM) direct current. The device presented in Publication IV utilises inductively coupled wireless power transfer to provide all the needed power for SMA actuation for the locomotion of the robotic caterpillar.

\subsubsection{Passive Wireless Sensors}

Passive wireless sensors are devices that enable measurement and wireless interrogation without a dedicated power source on the sensor side, nor energy harvesting from the ambient environment. Considering long-range sensors, of the order $1 \mathrm{~m}$ and greater, there are three primary types; Surface Acoustic Wave (SAW) sensors, RFID sensors and harmonic sensors. These are well described and compared in the doctoral thesis [59] and will be here summarised, and updated with recent developments in the field.

SAW sensors are based on the conversion of the reader's incident electromagnetic wave into a mechanical wave by exploiting the piezoelectric effect of an interdigital transducer. The acoustic wave is propagated to, and reflected from, the SAW sensor and converted back to an electromagnetic signal at the antenna. Identification is achieved by properly positioning the reflectors on the surface for modulating the timing of the reflected signal. The SAW signal can be modified by a number of sensed properties including temperature, pressure, strain, humidity, bio sensing and gas sensing ${ }^{[60]}$. SAW devices can operate on any frequency, but ISM $433 \mathrm{MHz}, 868-930 \mathrm{MHz}$ and $2.45 \mathrm{GHz}$ are common in the literature [61]. Readout ranges of SAW sensors are limited by permissible power in SAW frequency bands. In Europe $25 \mathrm{~mW}$ is permitted at $2.45 \mathrm{GHz}$ and 433 $\mathrm{MHz}$, enabling $36 \mathrm{~cm}$ and $2.5 \mathrm{~m}$ ranges respectively ${ }^{[62]}$. However, using a $4 \mathrm{~W}$ source readout distance of $13 \mathrm{~m}$ at $2 \mathrm{GHz}, 26 \mathrm{~m}$ at $1 \mathrm{GHz}$ and $51 \mathrm{~m}$ at $500 \mathrm{MHz}$ is calculated theoretically ${ }^{\left[6_{3}\right]}$.

Ultra High Frequency (UHF, 865-868 MHz and 902-928 MHz) RFID is a technology that is by now standardised [64] and is widely applied for identification and tracking in logistics, medical care, transport, agriculture and inventory management ${ }^{[65]}$. Passive UHF RFID operates based on the RF to DC conversion of the incoming radio signal, essentially energy harvesting from the incident RF signal, for powering of the tag IC. The tag response is achieved by the modulation of the backscattered signal by switching the tags input impedance [66]. UHF RFID is now evolving beyond supply chain management to sensor networks [67] and the integration sensing functionality has been an area of research for over a decade ${ }^{[68-71]}$. Early works apply a Wireless Identification and Sensing Platform (WISP) approach that involves RF energy harvesting and storage in the tag over a long period to enable burst measurements ${ }^{[68,69]}$. The latter work suggests a maximum readout distance of $2 \mathrm{~m}$. More recently, energy harvesting base approaches have achieved readout distances of $8 \mathrm{~m}^{\text {[72] }}$ and 10-12 $\mathrm{m}$ [73]. In addition, numerous approaches for loading the modulation impedance of a UHF 
RFID antenna are emerging [74], with potential to achieve similar readout distances.

Harmonic sensors utilize the frequency conversion of incident electromagnetic signal(s) using a non-linear element ${ }^{[75]}$. In one realisation a single fundamental frequency is transmitted and harmonics are reflected at multiples of the frequency [76], an approach that brings challenges in sensor antenna bandwidth and often violates usable electromagnetic spectrum restrictions. A more practical alternative is the transmission of two fundamental frequencies, and reflection of multiples of their sum and difference frequencies. The 'Zero Power Sensor Network' ${ }^{[77]}$ forms the direct background to the work of this thesis. It takes the latter approach, sending two frequencies in the UHF ISM band, $865 \mathrm{MHz}$ and $867 \mathrm{MHz}$. It has been demonstrated up to $11 \mathrm{~m}$ readout range. Any sensor element, which manifests its measurement as a change from $1 \mathrm{~s}-10 \mathrm{sF}$ can be measured with this technique. The platform has been applied for applications in temperature, accelerometer and biomedical measurements [77].

The work of this thesis builds on the direct background by integrating the reader device, presented in Publications V and IX, and integrating UHF RFID for activation and identification of passive wireless sensors presented in Publication VIII.

\subsubsection{Swallowable Capsules}

The state of the art in swallowable capsule technology is described in detail in Publication I of this work. This review paper will be here summarised and supplemented with developments in the field since its publication.

The first swallowable capsule device, measuring core temperature was described in the literature in $1957^{[78]}$. However, it was not until the 1990 s when advances in semiconductor technology enabled the development of a multitude of devices that were sufficiently miniaturised to be practical for human ingestion. There are a number of commercial swallowable devices, many of which have a primary function of capsule endoscopy [79]. Multisensing capsule devices have emerged to measure temperature, pressure and $\mathrm{pH}$ within the gut environment. Further, electromagnetic actuation enables smart capsule for targeted drug delivery. Commercial capsules measure 9-13 mm in diameter and 23 - 32 $\mathrm{mm}$ in length. They are largely battery powered, though some capsules with inductive powering exist.

In addition to commercial devices, numerous research efforts continue on various aspects of swallowable capsule technology. Integrating robotics with swallowable devices seeks to control the locomotion of the capsule through the gut. Efforts aiming to track capsule location are numerous, and include RF triangulation, radioactive tracer detection, or emission of ultrasonic waves from within the body ${ }^{[80]}$. Measurement of capsule transit time in the oesophagus, using Micro-electromechanical System (MEMS) accelerometers, has also been developed ${ }^{[81]}$. A number of ambitious projects have attempted to make a leap in swallowable capsule technology. One example, the IDEAS (Integrated Diagnostics for Environmental and Analytical Systems) project ${ }^{[82]}$, has applied system-on- 
chip technology to integrate classical sensors (temperature, conductivity and $\mathrm{pH}$ ) along with lab-on-chip technology to include chemical biosensors. Another ground-breaking project, VECTOR (Versatile Endoscopic Capsule for Gastrointestinal Tumour Recognition) has worked towards integrating complex microrobotics with endoscopic imaging. The project has developed capsule manipulators for tissue sampling and treatment along with retractable legs to control movement.

Given Imaging PillCam ${ }^{\circledR}$ has remained a purely endoscopic capsule and has gathered significant traction in the market, with more than 2 million patients and 1,900 clinical studies by 2018. Research has gathered pace, and a multitude of innovative capsules are detailed in recent literature ${ }^{[83]}$. In addition to classical measurement previously mentioned, new developments include gas sensing, visible and IR spectroscopy, florescence endoscopy, electrochemical sensing (work of this research) and ultrasound imaging which have been implemented and tested in the laboratory. Concept level research for future implementation in swallowable form includes pyroelectric measurements, Raman spectroscopy, physisorption sensors and surface acoustic wave sensors.

The swallowable capsule presented in Publication II complements the state of the art by adding a swallowable device with electronic tongue sensor capable of performing electrochemical analysis of the gastrointestinal track in vivo. 


\section{Wireless Sensor Systems}

\subsection{Swallowable E-tongue Capsule}

The human gastrointestinal (GI) tract has remained largely undiscovered. Traditional investigation methods have allowed investigation of the stomach (gastroscopy) and large intestine (colonoscopy), at much discomfort to the patient and cost to the health system. The small intestine has remained largely inaccessible to these traditional methods. As described in Section 2.3.7 and Publication I, ingestible devices are emerging and allowing investigation of the whole GI tract, including the small intestine. The work of this thesis, described in this section, progresses beyond the state of the art by adding a swallowable device with electrochemical electronic (E)-tongue sensor, with potentiostatic voltammetry readout mechanism for the detection of non-specific markers of ulcerative colitis and Crohn's disease.

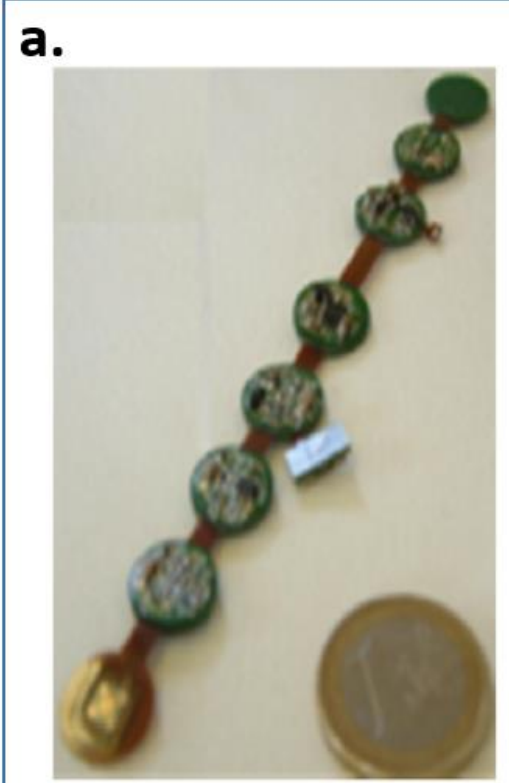

b.

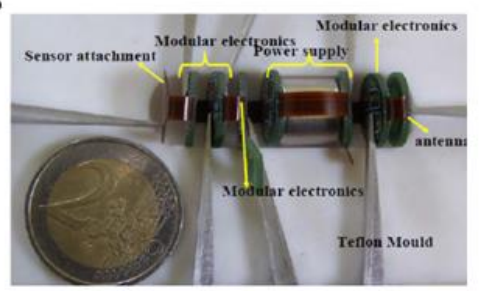

C.

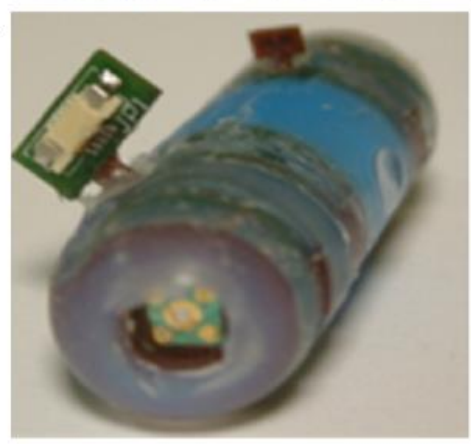

Figure 5. Swallowable E-tongue capsule device. (a) Electronic system implementation on rigidflex PCB. (b). Pleat folded electronic system with battery integration. (c) PEEK encapsulated electronic system, E-tongue sensor at the front, extruding programing connector and activation tab. 
The swallowable E-tongue system developed in this work is directly based on a system that was developed for liquid analysis with applications in the food and pharmaceutical industries in mind [84]. The capsule's predecessor employed a macro scale E-tongue sensor with benchtop readout device and wired PC user interface. The advancements on this background work are described in this section and thesis Publication II.

The implementation of the swallowable E-tongue capsule was achieved using a rigid-flex structure with modular electronics on 12 mm FR4 disks interconnected on a flexible polyimide substrate (Figure $5 \mathrm{a}$ ). This implementation allowed the folding of the system in a pleat fashion (Figure $5 \mathrm{~b}$ ) to be encapsulated within a cylindrical volume of $12 \mathrm{~mm}$ diameter and $28 \mathrm{~mm}$ in length. The encapsulation of the device (Figure 5c) was carried out in a polyether ether ketone shell (PEEK) which is biocompatible and widely used for in vivo implant applications.

\subsubsection{Requirements and Constraints}

The maximum transit time of the GI tract is 72 hours. For hygiene and practicality reasons the capsule can be considered disposable, so 72 hours is considered as the full lifetime of the device. A measurement interval of 2.5 mins was specified as appropriate for the application, considering the slow movement of the GI tract. A measurement cycle consisted of $28 \mathrm{~s}$ of cyclic voltammetry, $1 \mathrm{~s}$ of wireless transmission and 2 minutes of sleep mode. The wireless transmission scheme would need to achieve a reliable readout at a distance of $2 \mathrm{~m}$ so that the patient could conveniently shower, sleep etc. without being inconvenienced by the reader. The wireless transmission would need to be designed at a frequency low enough to penetrate human tissue but high enough that the antenna could be sufficiently small for capsule integration. The transmission would need to be robust in the environment of human tissues and robust to changes in the capsules dielectric environment.

In addition to the power and communication requirements, there were numerous additional challenges relating to miniaturisation, biocompatible encapsulation, sensor to gut fluid direct access, sensor integration, stability and cleaning. These are considered outside the context of this thesis and are briefly described in Publication II, and further elaborated in ${ }^{[85]}$ and ${ }^{[86]}$.

\subsubsection{System Level Design}

A block level description of the capsule device is shown in Figure 6a. At its front end, the capsule device comprises the E-tongue sensor with direct access to GI tract fluids, stimulated through an analogue potentiostat with current acquisition, amplification and filtering circuit, elaborated in Section 3.1.3. The system processing device is a $\mathrm{PIC} 18 \mathrm{~F} 2520$ microcontroller. Wireless communication is realized with an RF transceiver device and antenna, elaborated in Section 
3.1.4, and powering is provided through a power conditioning circuit from a battery described in Section 3.1.5. Referring to Figure 6, each component block occupies roughly one circular module in the substrate. From front to back in Figure 5a; E-tongue sensor, current acquisition and amplification, sensor control, microcontroller, power conditioning (split over two modules with battery connection sides on the back side), RF transceiver and finally the loop antenna.

On the receiver side (Figure 6b), a dipole antenna receives the capsule transmission and couples it to an RF transceiver for demodulation. The same PIC18F2520 microcontroller performs the processing in the receiver. The receiver is mains powered and interfaced via a Universal Serial Bus (USB) to serial converter to the PC where a LabVIEW user interface performs visualisation and storage for later analysis, of the capsules voltammetry data.

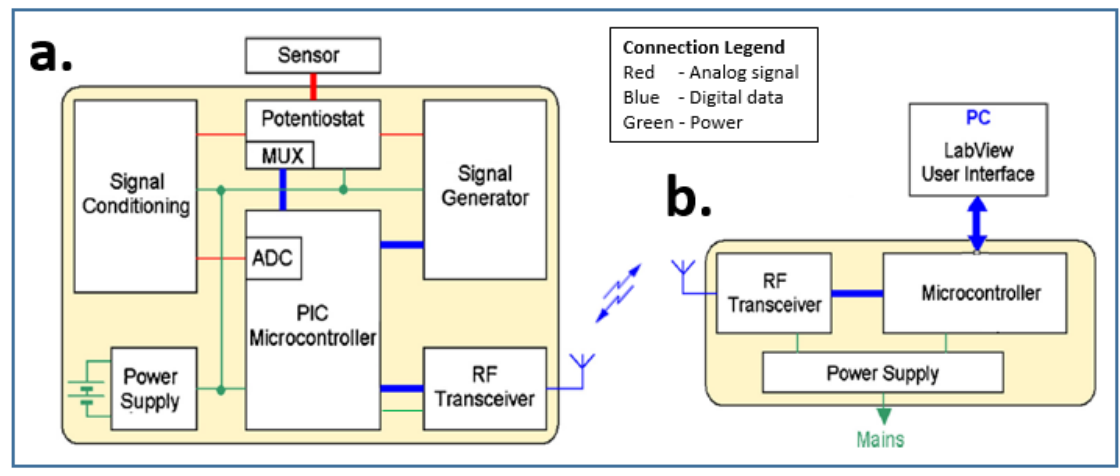

Figure 6. Block diagram of swallowable capsule and receiver module. (a) Swallowable capsule device with sensor control and readout, wireless transmitter and power supply. (b) Receiver module with wireless receiver, PC user interface and mains connected power supply.

\subsubsection{E-tongue Sensor and Analogue Front End}

Analytical electrochemical sensing is based on the measurement of oxidisation and reduction current, and their related cell voltages, of ionic compounds in an electrochemically active liquid at a metal electrode surface. A potentiostat circuit forces a cell voltage across a counter and reference electrode and measures the cell current from a working electrode. The E-tongue sensor comprises four working electrodes of differing noble metals (gold, platinum, iridium and rhodium) to exploit the different electrochemical behaviour of various metal-liquid interfaces. The working electrodes surround a large central gold counter electrode and a silver silver-chloride reference electrode. The cell voltage is forced across the reference and counter electrodes while the required current is provided through the counter to maintain the cell potential. This current is extracted from the working electrode, held at virtual ground.

In the swallowable capsule case, a miniaturised E-tongue sensor was fabricated on a 6x6 mm chip (Figure7a) and assembled on the capsule polyamide substrate using a flip chip over hole process with anisotropic conductive adhesive (Figure7b). The sensor was stimulated with cyclic voltammetry through a 
potentiostat circuit, and cell current is acquired through a transimpedance amplifier. A variable gain stage is included with a microcontroller auto ranging algorithm, before the signal is level shifted to the measurement range of the microcontroller as shown in (Figure7c). Detailed description of the sensor, assembly and readout electronics are given in Publications II and VII and in our other published works ${ }^{[84-86]}$. The power consumption of the whole analogue front end of the discrete potentiostat in Publication VII was measured to be $26 \mathrm{~mW}$ ( 8.8 $\mathrm{mA}$ at $3 \mathrm{~V}$ ). The integrated device implemented in Publication VII consumed close to $100 \mathrm{~mW}$, as it was implemented on a non-power optimised Application Specific Integrated Circuit (ASIC).

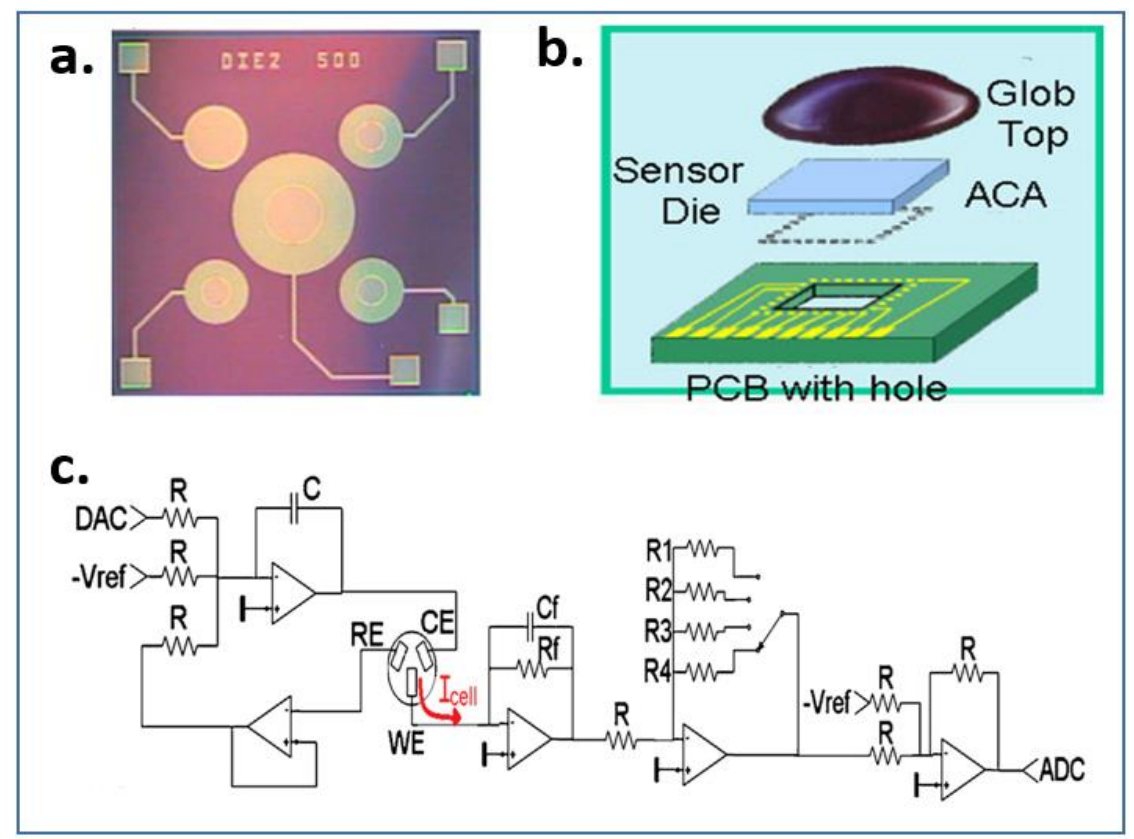

Figure 7. E-tongue sensor and readout electronics. (a) Electronic tongue sensor, fabricated on a $5 \mathrm{~mm} \times 5 \mathrm{~mm}$ die. (b) Flip-chip-over-hole assembly process. (c) Potentiostat sensor control forcing required current $I_{\text {CELL into }} C E$ (counter electrode) maintain cell voltage (DAC - $V_{\text {REF }}$ ) between the reference electrode (RE) and CE, and current acquisition (from working electrode (WE)) amplifier variable gain stage, and level shifter to ADCs positive range.

\subsubsection{Radio Architecture}

The ISM frequency band at $433 \mathrm{MHz}$ carrier was selected as a good trade-off between electromagnetic penetration through human flesh, propagation range in free space, practical antenna dimensions, power consumption and for the availability of transceiver components off the shelf. A Melexis transmitter receiver pair (TH72015/TH71102) was selected to implement a Frequency Shift Keying (FSK) modulation that can achieve $40 \mathrm{kbps}$. FSK was selected due to its simple and low power implementation in the transmitter and immunity to amplitude variations in the received signal. The transmitter provides single ended 
output at powers of -12 to $+10 \mathrm{dBm}$ with corresponding current consumptions of 2.5 to $10.6 \mathrm{~mA}$ at $3 \mathrm{~V}$ (7.5 to $31.8 \mathrm{~mW}$ ). A $12 \mathrm{~mm}$ diameter loop antenna was used, well below the optimal quarter wave size for this frequency (173 $\mathrm{mm}$ ). This is justified based on the size constraints applied by the capsule dimensions. The antenna was Q-degraded with a shunt resistor to make the resonance matching more robust to impedance variation due to changes in the dielectric in its environment as the capsule transits through the GI tract. A serial byte Manchester encoding algorithm was used, to enhance immunity to decoding errors, with a data rate of approximately $1 \mathrm{kbps}$. On the receiver side a double conversion superheterodyne receiver was used with a phase co-incidence demodulator to achieve a sensitivity of $-107 \mathrm{dBm}$. A standard dipole antenna was included in the receiver as the need for miniaturisation was less intensive in this case. Details of the capsule radio architecture are provided in Publication VI.

\subsubsection{Power Supply}

Current consumption of the capsule was measured to be $117 \mathrm{uA}, 10.5 \mathrm{~mA}$ and 9.5 $\mathrm{mA}$ in sleep, measuring and wireless transmission modes respectively giving power consumptions of $350 \mathrm{OW}, 31.5 \mathrm{~mW}$ and $28.5 \mathrm{~mW}$. The capsule performed voltammetry measurements for a maximum of $28 \mathrm{~s}$, wireless communication for $1 \mathrm{~s}$ and spent 2 minutes in sleep mode, giving a cycle time of 2.5 minutes. Taking the maximum measurement frequency of 2 minutes gives an average current consumption of $2.12 \mathrm{~mA}$ and a power requirement of $152 \mathrm{mAh}$ for 72 hours measurement. A single $3 \mathrm{~V}$ LiMnO2 2L76 cell was selected, providing 165 $\mathrm{mAh}$ in an $11.4 \mathrm{~mm}$ diameter and $10 \mathrm{~mm}$ length package. A switched capacitor converter was used to maintain the system voltage at $3 \mathrm{~V}$, providing the advantage of smaller switching components, and higher efficiencies at low currents over typical inductor based converters. A charge pump inverter was used to generate the negative voltage, and low drop out regulators to provide stable and low noise positive and negative voltages required for the bipolar analogue potentiostat circuit.

\subsubsection{Test and Evaluation}

To evaluate the radio architecture, a test setup was devised which involved placing the capsule central in a standard water cooler Plexiglas container, 18.9 $\mathrm{l}$ with $27.3 \mathrm{~cm}$ diameter and $49.5 \mathrm{~cm}$ height, filled with $1.5 \mathrm{~g} / \mathrm{l} \mathrm{NaCl}$ dissolved in purified water. Such a saline solution provides a good representation of the conductivity (represented by the $\mathrm{NaCl}$ ) and dielectric constant (represented by the water) of human tissue. The evaluation was conducted for transmission in air (empty container) and in the described saline solution with the transmitting antenna positioned approximately centrally in the container. As the loop antenna generates a highly directional field, the test was conducted for the best (antenna plane normal to direction of transmission) and worst (antenna plane perpendicular to direction of transmission) cases. The transmitter was set to output $+10 \mathrm{dBm}$ and continuously alternated between 1 and o transmissions at approx- 
imately $1 \mathrm{kHz}$. The signal was received by (a) an Anritsu MS2711D spectrum analyser and (b) the TH71102 receiver, each coupled to a dipole antenna. The transmitter and receiver were placed at table height $(\sim 1 \mathrm{~m})$, in a large room without obstruction in the range of 5 wavelengths $(3.5 \mathrm{~m})$ from transmitter or receiver. The transmitter, placed within the Plexiglas container remained stationary while the receiver was moved in steps of $0.1 \mathrm{~m}$ at increasing distances from $0.5 \mathrm{~m}$ to $2.5 \mathrm{~m}$.

The results, shown in Figure 8, demonstrate the dependence of the received signal strength with distance. In both cases the measurements were taken in what was considered to be the best and worst cases in terms of the alignment of the transmitting antenna with respect to the receiving antenna. If the transmitter antenna is an ideal electrically small loop, the worst propagation case is normal orientation, where the plane of the loop is 'facing' the receiver and the best case is perpendicular orientation where the plane of the loop is turned $90^{\circ}$ from normal. In the case of the spectrum analyser receiver (Figure 8a) the received signal is observed to reduce by $0.7 \mathrm{dBm}$ per $\mathrm{cm}$ in air in an approximately linear fashion. An unusual observation is made, that the normal and $90^{\circ}$ orientations propagate roughly equally in air, demonstrating that the antenna does not behave as an ideal loop, but rather some combination of a loop (magnetic dipole) and an electric dipole. This is proposed to be due the Q-degrading resistor. This results in electric fields being stimulated in the antenna creating a radiation pattern that is expected to be some superposition of magnetic and electric fields. The addition of the saline solution increases the attenuation by $7 \mathrm{dBm}$ in the best case $(\mathrm{d}=0.5 \mathrm{~m}$ antenna normal direction) and $18.5 \mathrm{dBm}(\mathrm{d}=2 \mathrm{~m}$, antenna perpendicular direction). Considering the receiver sensitivity of $-107 \mathrm{dBm}$, the results of Figure 8a show a reliable connection with overhead of $18 \mathrm{dBm}$ in the worst case. The results also highlight the degrading of the antenna performance in the saline solution with $7 \mathrm{dBm}$ difference between normal and perpendicular directions in the worst case. From this, it is clear that the propagation mode is altered by the saline solution, and the ratio of magnetic and electric propagation modes changes. This is likely due to matching effects of the conductive medium near the antenna. For receiver verification, the TH71102 Received Signal Strength Intensity (RSSI) output was examined and is shown in Figure 8b. The results show a decline of $300 \mathrm{mV}$ per meter in RSSI and a difference of $400 \mathrm{mV}$ between air transmission and saline transmission in the worst case $(\mathrm{d}=1.3 \mathrm{~m}$, saline solution, antenna perpendicular direction). In addition, the wireless communication was tested in an $8.5 \mathrm{~g} / \mathrm{l} \mathrm{NaCl}$ solution representing a conductivity beyond worst case in human tissue. The results, in Publication VI, demonstrate an effective connection up to $2.5 \mathrm{~m}$ with an overhead of $7 \mathrm{dBm}$ in the worst case. 


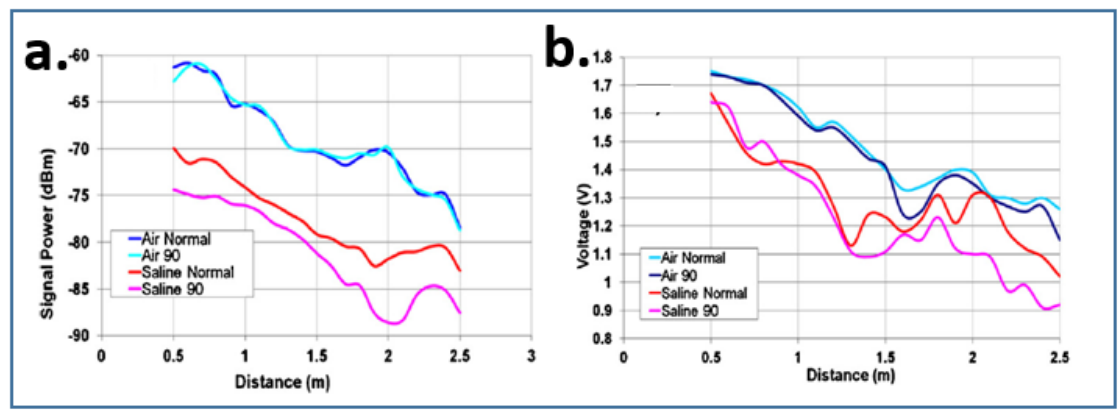

Figure 8. Radio range tests, capsule inside saline vessel with $1.5 \mathrm{~g}$ sodium chloride / litre tap water. Best (normal orientation) and worst (90 rotation from normal) case orientations of the loop transmitter with respect to the receiver (a) Signal power received at Spectrum Analyser with dipole antenna. (b) RSSI at Melexis receiver.

The following tests of the potentiostat readout circuit were carried out in a sulphuric $\left(\mathrm{H}_{2} \mathrm{SO}_{4}\right)$ acid regent of analytical purity (95- $\left.98 \%\right)$ diluted in purified water to $0.1 \mathrm{M}$ concentration. Cyclic voltammetry was performed between $\mathrm{o}$ $1.5 \mathrm{~V}$ at a scan rate of $200 \mathrm{mV}$ per second. To benchmark the developed potentiostat, a comparison under the same conditions with a laboratory CHI66oB potentiostat was conducted with the macro E-tongue sensor (as described in [84]) and commercial $\mathrm{Ag} / \mathrm{AgCl}$ reference electrode. The results, illustrated in Figure 9a show the cyclic voltammograms generated by the developed potentiostat compared with those from the commercial device. The results show expected oxidisation and reduction peaks, with a systematic offset of $-100 \mathrm{mV}$ in the developed device most likely due to inaccuracy in the calculated vs real voltage applied that can be removed by calibration. The developed potentiostat generates a well-defined and low noise voltammogram that compares well with the commercial device.

The selected battery was tested simply by loading the battery with a large resistor to represent the sleeping power drain of $350 \mathrm{uW}$. Considering an average operation voltage of $2.8 \mathrm{~V}$, and $24 \mathrm{k} \Omega$ resistor across the battery drained an average continuous current of $117 \mathrm{uA}$. The measurement and transmitting cycles were combined, and considered to consume the maximum power of $32 \mathrm{~mW}$. Considering the $2.8 \mathrm{~V}$ battery average, a $261 \Omega$ resistor was used to drain an average of $10.7 \mathrm{~mA}$ from the battery. To emulate capsule operation the $261 \Omega$ resistor was switched across the battery for a period of $60 \mathrm{~s}$ at intervals of $150 \mathrm{~s}$. The battery was found to provide enough energy for approximately 80 hours of operation in the described test, allowing the 72 hour operation requirement with just over $10 \%$ overhead.

To verify the whole system operation, the capsule device was tested over the 72 hour measurement period, battery powered and with wireless communication at a nominal distance selected as $1.5 \mathrm{~m}$. The same test conditions as described above were used. The results, in Publication II, showing 500 overlaid voltammograms, demonstrate effective operation of the whole system. The voltammogram shows no measurement inconsistency and stability of the oxidisation and reduction peaks, and a noise based accuracy of $\pm 0.5 \mathrm{uA}$ corresponding 
to a Signal to Noise Ratio (SNR) of 6o, based on reduction peak of $30 \mathrm{uA}$. Further tests were conducted using the integrated, (gold only) electrode sensor described in Section 3.1.3. In this case, one of the gold working electrodes is used as a pseudo reference in place of the more stable $\mathrm{Ag} / \mathrm{AgCl}$ standard. 50 cyclic voltammograms were conducted under the same conditions above described, except the potential window was narrowed to $0-1.1 \mathrm{~V}$. The generated voltammograms, shown in Figure 9b still show clearly defined voltammograms with expected oxidisation and reduction peaks. However the measured currents are somewhat reduced, due to smaller electrode surface area and therefore the noise levels are increased. A drift along the voltage access of approximately $50 \mathrm{mV}$ was observed due to intrinsically less stable pseudo reference.

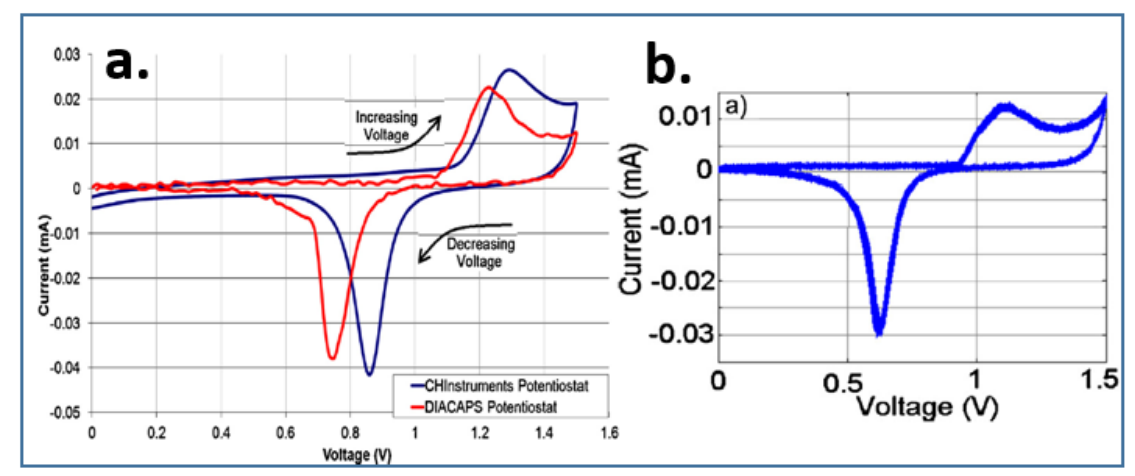

Figure 9. System tests, cyclic voltammograms in Sulphuric Acid. (a) Benchmarking test of developed potentiostat against $\mathrm{CH}$ instruments laboratory potentiostat with integrated $\mathrm{E}$-tongue sensor and commercial $\mathrm{Ag}-\mathrm{AgCl}$ reference. (b) Full wireless battery powered system cycle tests, 500 overlaid cyclic voltammograms taken at 10 minute intervals using planar E-tongue sensor and commercial $\mathrm{Ag}-\mathrm{AgCl}$ reference.

\subsubsection{Summary}

A swallowable capsule with electrochemical E-tongue has been developed and shown to perform effectively when compared with a $\mathrm{CH}$ Instruments commercial device. The device included a $433 \mathrm{MHz}$ wireless communication link which was shown to establish a reliable connection to a readout distance up to $2.5 \mathrm{~m}$ when submerged in a saline solution that models the in vivo environment. Due to the short measurement lifetime of the capsule, and disposable nature of the application, energy autonomy was accomplished through the use of a high capacity $\mathrm{LiMnO}_{2}$ battery, which allowed $28 \mathrm{~s}$ voltammetry measurements to be taken at intervals of 2 minutes for the 72 hour lifetime required by the application. 


\subsection{Power Autonomous Condition Monitoring}

Industrial internet is a key driver in the adoption of IoT technologies. Online condition monitoring of existing industrial infrastructures presents the opportunity to generate significant value in process control, increased efficiency and enhanced safety. The petrochemical industry, where flammable liquids and gasses are pumped at high pressures over significant distances, is considered a safety critical application where valve deterioration leading to leakage can present potentially catastrophic consequences. Further, the leakage of such assets can bring costs to infrastructure operators and expel toxic pollutants into our environment.

The work of this thesis, summarised in this section and detailed in Publication III and live-demonstrated in [4], presents the development of a system for petrochemical valve defect detection. The system developed direct background work including a novel MEMS based acoustic emission sensor ${ }^{[87]}$, a novel ultralow power wake-up radio technology ${ }^{[88]}$ advancing the state of the art described in Sections 2.3.2. Energy harvesters on both the sensor and base station side complement the state of the art in Section 2.3.4. delivering a fully power autonomous solution.

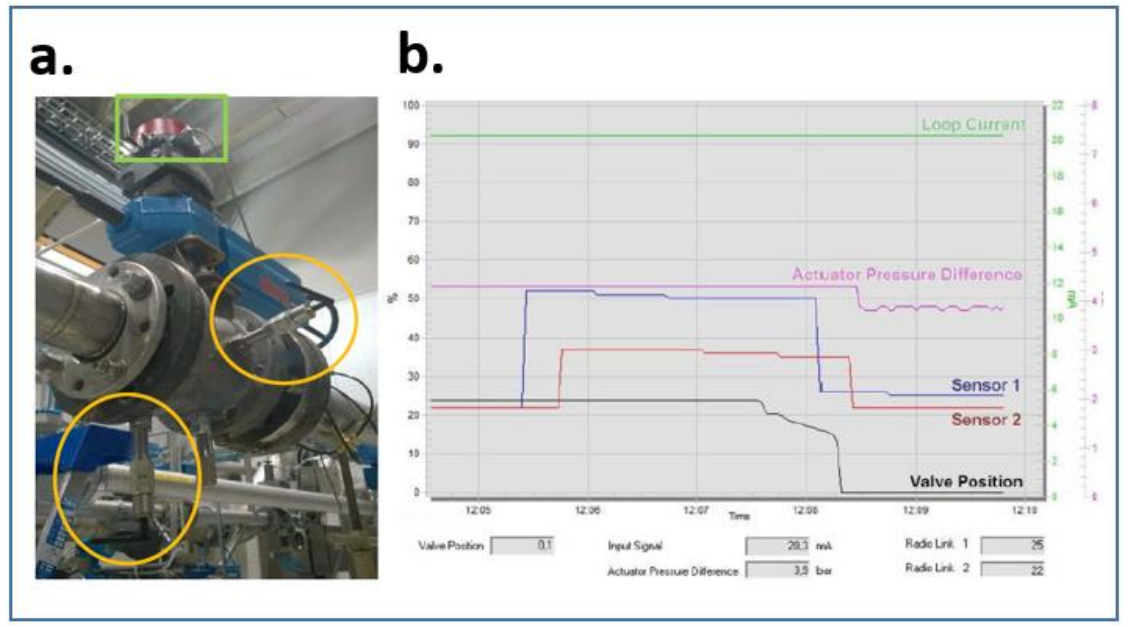

Figure 10. Photo of the deployed valve leakage detection system, and valve leakage readout. (a) Showing two bolt integrated sensor devices (yellow circles) and Metso VG9000 valve controller integrated base station (green rectangle) and (b) Metso Field Care visualisation of valve leakage measurement with actuator pressure difference (pink), sensor 1 measurement (blue), sensor 2 measurement (red) and valve position (black).

The monitoring system comprised a sensing device, including a thermal energy harvesting realisation. The sensors were integrated into bolt fixtures for seamless deployment to petrochemical valve junctions, piloted with our industrial partner Metso Flow Control Oy, as shown in Figure 10. Two bolt integrated sensors are shown in Figure 10a (yellow circles), placed at perpendicular angles 
to the valve junction. The base station, which asynchronously controlled the measurement process, was integrated into a Metso VG9ooo valve controller, as shown in the green rectangle in Figure 10a. The base station included an industrial current loop energy harvester. Data transfer was made to the Metso Field Care platform for onward interface to the cloud. Figure 1ob shows the readout of the pilot setup in the Metso Field Care environment where test valve with introduced leakage defect was investigated.

\subsubsection{Requirements and Constraints}

Existing valve monitoring entails manual maintenance on an annual or biannual basis. Therefore, to present a significant advantage the system lifetime needed to be continuous or in the order of many years. Therefore, power autonomy was considered an essential requirement to allow continuous operation. Valve deterioration occurs in timescales of months to years. Therefore a long measurement interval in the order of days is a key requirement. In this case many of the standard radio protocols, based on regular connection intervals, mentioned in Section 2.3.1 were considered unsuitable. An asynchronous wakeup radio was required to allow measurement of the valve deterioration at long intervals in case of normal operation, and at short intervals if some deterioration is detected.

The developed system would be required to detect the acoustic emissions generated by micro-fractures at the valve junction, therefore the sensor would need to be positioned such as to establish a good acoustically conductive path to the point of potential micro-fractures. The system needed to be wireless, and seamlessly deployed to allow easy retrofitting to existing infrastructures. Due to the dangerous nature of the potential for gaseous combustibles in the deployment environment, the system had to be designed to be intrinsically safe according to the ATEX-II T6 (Appareils destinés à être utilisés en ATmosphères Explosives) standard ${ }^{[89]}$ for intrinsic safety.

\subsubsection{System Level Design}

A block level description of the system is illustrated in Figure 11. The bolt sensor, represented in Figure 11a, shows the MEMS leakage sensor and readout electronics (detailed in Section 3.2.3) placed at the valve interface, perpendicular to the direction of flow across the valve. The thermoelectric element is included close to the valve interface (described in Section 3.2.5). The sensor's system had dual radios; the wake-up signal detector (described in Section 3.2.4) and standard active radio. The base station, shown in Figure 11b, included a standard active radio and a power amplified wake-up signal generator. An industrial current loop energy harvester was included to make the system fully power autonomous. 

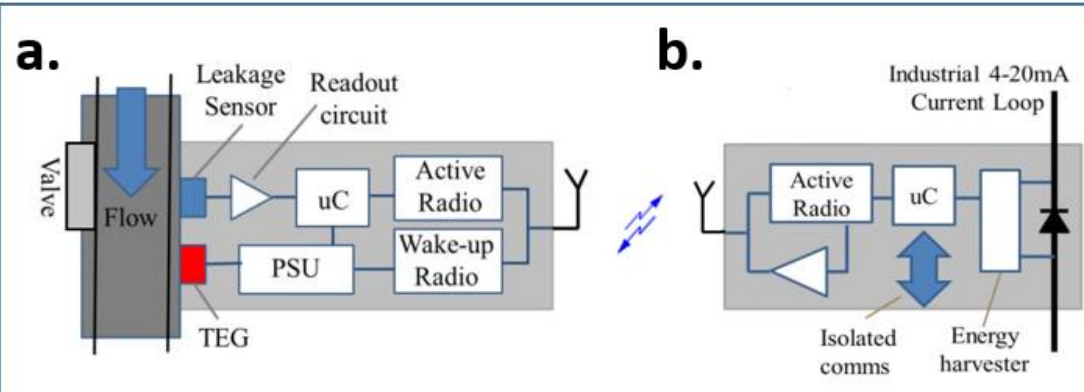

Figure 11. Block diagram of power autonomous valve leakage detection system. (a) Bolt integrated sensor device with MEMS acoustic emission sensor and readout circuit, TEG and power conditioning, microcontroller core, dual radio channels and external antenna and (b) Valve controller integrated base station with current loop energy harvester, microcontroller, magnetically isolated communication, dual radio channels and external antenna.

\subsubsection{Acoustic Emission Sensor}

The feasibility of acoustic emission sensors for valve leakage detection is presented in related literature [90], where the acoustic emissions are shown to be a function of defect size and valve pressure. The device of Publication III, fabricated on a Silicon on insulator (SOI) process, was essentially a unidirectional accelerometer designed to detect vibrations normal to the surface on which it's mounted. The sensor measures $1.8 \times 1.8 \times 0.8 \mathrm{~mm}^{3}$ and was scaled to a resonant frequency of $110 \mathrm{kHz}$ where valve micro fracture acoustic emissions are detected in the application. A cross section of the fabricated device is illustrated in Figure 12a. The bottom plane was mounted to the detection surface, and the upper electrode provided a proof mass allowed to vibrate at the resonant frequency. Further details of the sensor are provided in Publication III and [87]. The presented MEMS based acoustic emission sensor offers advantages over piezoelectric devices in terms of small size, mass manufacturability and related cost reduction.

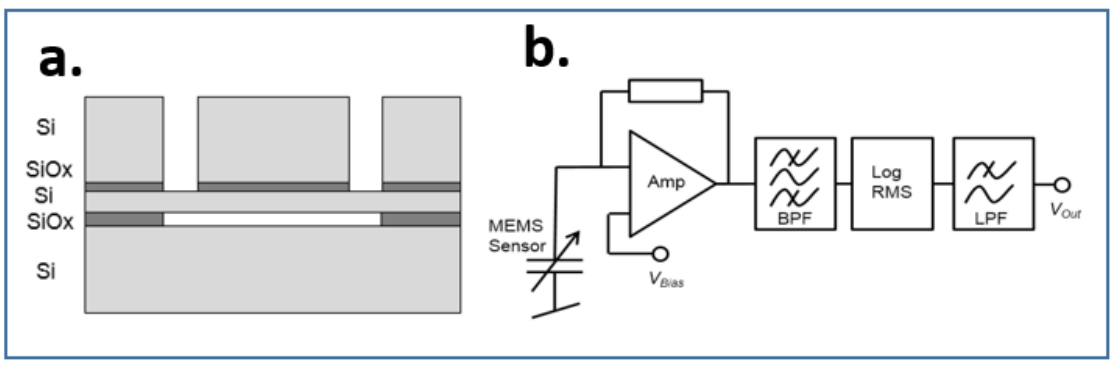

Figure 12. MEMS acoustic emission sensor and readout circuit. (a) Fabricated MEMS acoustic emission sensor with SOI process and (b) MEMS sensor represented as a variable capacitance, biased transimpedance amplifier, band pass filter, logarithmic RMS converter and low pass filter.

The sensor's measurement manifested as a varying capacitance between the upper proof mass electrode and the mounted bottom plane electrode. The readout electronics, represented in Figure 12b, were designed to detect this 110 
$\mathrm{kHz}$ variation and convert it to a DC Root Mean Squared (RMS) value representing the magnitude of AE. The signal was acquired through a DC biased low noise transimpedance amplifier and band pass filtered at $110 \mathrm{kHz}$. The signal was integrated in the logarithmic amplifier, with gain such as to provide the RMS value of acoustic emissions. Finally, a low pass filter removes signal harmonics and high frequency noise to provide a stable output DC voltage.

\subsubsection{Radio Architecture}

The system's radio architecture included a two channel system; the unidirectional wake-up radio and bidirectional active radio for data transfer as shown in Figure 13a. Both radios were designed to operate in the ISM $2.45 \mathrm{GHz}$ band, selected as a balance between power requirement, antenna sizes and free space propagation range. $2.45 \mathrm{GHz}$ has a longer unobstructed propagation range than lower frequency ISM bands, and can be expected to perform better in industrial environments. Further, quarter wave dipole antennas, such as those used in WiFi trancievers are cheaply and readily available. A commercial Nordic Semiconductor transceiver is used on both base station and sensor sides. A Nordic proprietary protocol was used, as it offered the flexibility needed to directly encode a low frequency wake-up sub-carrier on the $2.45 \mathrm{GHz}$ carrier with very low latency when compared to standardised protocols like BLE and ANT.

The base station (top of Figure 13a) acted as master, with the ability to asynchronously initiate the measurement process via the wake-up radio. On the base station side two single pole double throw (SPDT) switches were used to switch between the active and wake-up radio paths. In the wake-up case the signal is switched through a $+20 \mathrm{dBm}$ power amplifier to amplify the $-10 \mathrm{dBm}$ transceiver output to $+10 \mathrm{dBm}$. The second SPDT coupled the signal to a quarter-wave monopole antenna for transmission. To produce the wake up signals, the base station generated a $120 \mathrm{kHz}$ subcarrier by modulating the $2.45 \mathrm{GHz}$ carrier generated by the transceiver as shown in Figure 13b. The wake-up signal is encoded by on/off modulating the subcarrier at a rate of $1 \mathrm{kbps}$ as shown in Figure 13b. The wake-up signal consisted of a 16-bit preamble and 8-bit wake up address allowing up to 256 sensor nodes to be addressed.

The sensor node (bottom of Figure 13a) acted as slave in all measurement processes. The two-channel radio was accomplished using a single antenna and a passive bidirectional coupler which presented an insertion loss of $-2 \mathrm{dBm}$ to the wake-up receiver, $-10 \mathrm{dBm}$ to the active radio and $28 \mathrm{dBm}$ of isolation between them. The wake-up signal detector based on the VTT patented wake up radio receiver is illustrated in Figure 13c. The circuit included a $2.45 \mathrm{GHz}$ matching circuit to resonate with the incoming signal and couple it to a HF detector diode. The diode performs an envelope detection function and was combined with a HF choke inductor that removed the $2.45 \mathrm{GHz}$ carrier signal. The $120 \mathrm{kHz}$ subcarrier resonated with the combination of this inductor and an input capacitor to a low frequency wake-up circuit, with a sensitivity of $100 \mathrm{uV}$, represented as a $2 \mathrm{M} \Omega$ resistance and a $10 \mathrm{pF}$ parasitic pad capacitance in Figure 13c. The low 
frequency wake-up IC included a 256-bit address correlator which eliminated the possibility of false wake-up. The described wake-up technique allowed passive down conversion of the $2.45 \mathrm{GHz}$ signal and resulted in a low power wakeup solution. In listening mode, the diode detector required $1 \mathrm{uA}$ of bias current and the low frequency wake-up IC consumed $5 \mathrm{uA}$. A total consumption of 6.8 uA was measured including $800 \mathrm{nA}$ of current leakage in the system. More detailed description of the wake-up infrastructure is provided in Publication III and ${ }^{[88]}$.

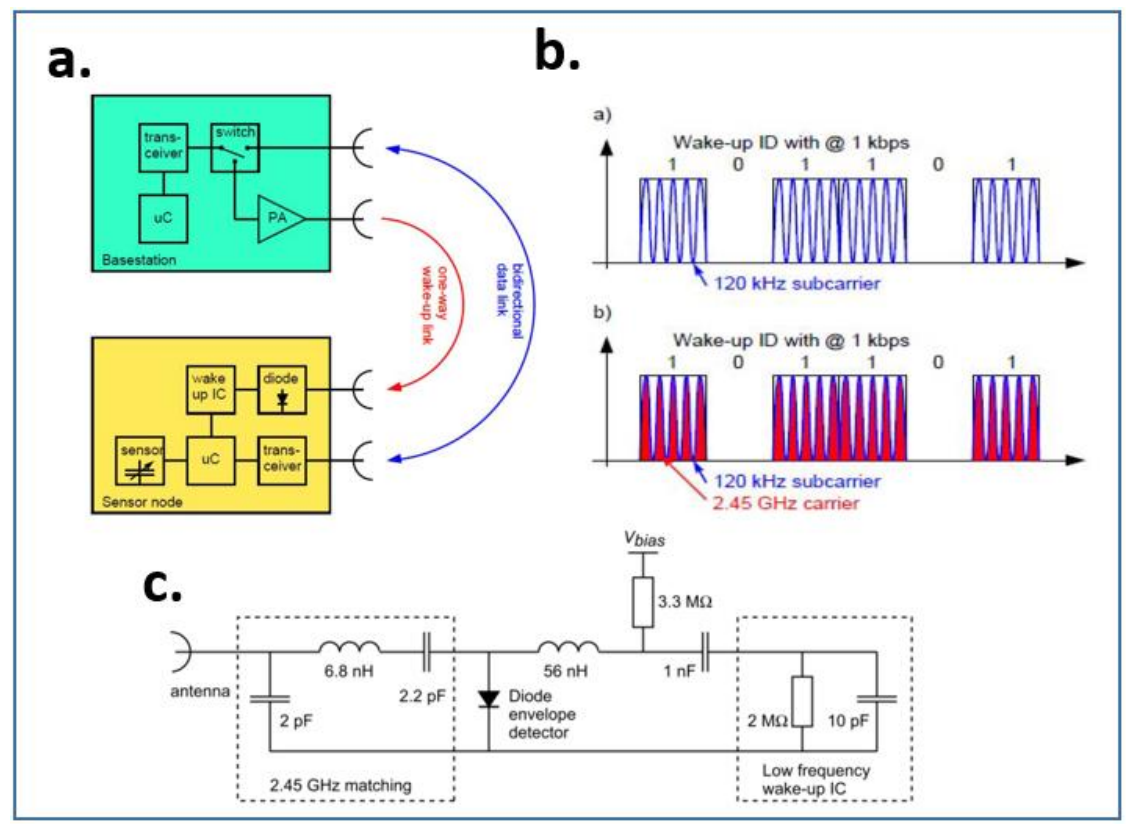

Figure 13. Wake-up radio, hardware architecture and wake-up signal generation. (a) Base station and sensor node with one-way wake-up and bidirectional data link. (b) Modulation of $1 \mathrm{kbps}$ wake-up ID on $120 \mathrm{kHz}$ subcarrier with $2.45 \mathrm{GHz}$ carrier. (c) Wake-up radio receiver with antenna, $2.45 \mathrm{GHz}$ matching circuit, diode envelope detector, low frequency matching, diode bias resister and low frequency wake-up IC.

\subsubsection{Power Supply}

Due to the long measurement interval of 12 hours, the energy consumption of the sensor node was dominated by the sleeping current of $6.8 \mathrm{uA}$. When a wakeup signal was received from the base station the sensor was activated on demand. The average active consumption, including; initialisation, measurement over 1 second window, radio communications and de-initialisation, was $63 \mathrm{~mA}$ over a time period of $6 \mathrm{~s}$. The active consumption of the sensor was $29 \mathrm{uW}$ when considered as the average over a 12 hour measurement interval. Nonetheless, the sensor node needed to be capable of both maintaining the $22 \mathrm{uW}(6.8 \mathrm{uA}$ at $3.3 \mathrm{~V}$ ) and needed to keep the wake-up radio in listening mode, and providing the $1.25 \mathrm{~J}$ ( $63 \mathrm{~mA}$ at $3.3 \mathrm{~V}$ for $6 \mathrm{~s}$ ) of energy to perform a measurement. 


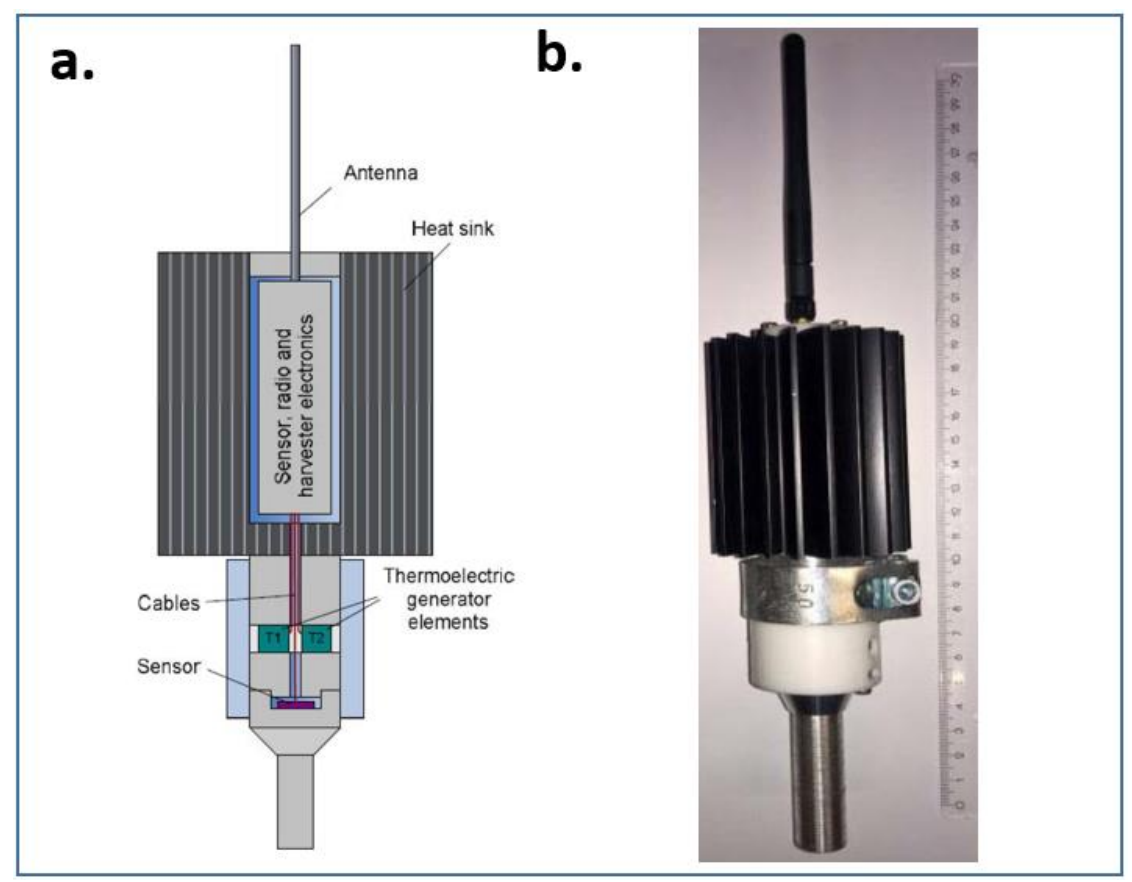

Figure 14. Thermal energy harvester for sensor power autonomy. (a) Block diagram showing bolt integrated sensor, TEG elements, electronics encased in heat sink and extruding antenna. (b) Photo of the realised energy harvesting bolt sensor.

To exploit a temperature difference between the pumped fluid and ambient air, a thermoelectric energy harvester realisation of the sensor bolt was developed. While the sensor device had to be glued to the bottom surface of the hollowed bolt, an off the shelf (Micropelt TGP-751) TEG element was inserted just above it. The energy harvesting metallic bolt provided an excellent heat conduction path from the valve fluid, enhanced by a brushing element, and cooled in the ambient air through a heat sink. The heat sink was hollowed, which directed heat conduction to its fins, and provided space for the sensor electronics and communication device to be placed as shown in Figure 14a. A plastic cap, with through-hole allowing the antenna connector to protrude, sealed the encasing. A Texas Instruments energy harvesting power controller (BQ25504) was used which included maximum power point tracking to optimise the power extracted from the TEG. A lithium polymer battery was used for energy storage and to ensure a stable and reliable source was continuously provided to the sensor electronics. A photograph of the energy harvesting bolt realisation is shown in Figure $14 \mathrm{~b}$.

To perform a measurement cycle, the base station needed o.15 $\mathrm{J}$ of energy (95 $\mathrm{mA}$ at for the $100 \mathrm{~ms}$ wake-up burst and $24 \mathrm{~mA}$ for a $1.5 \mathrm{~s}$ window for receiving of the node response) where the high current relates to the needed power amplifier for high power wake-up signal generation. The base station was integrated with the Metso VG9ooo valve controller that utilised a standard 4 - 20 
$\mathrm{mA}$ current loop for communication. To enable energy autonomy also for the base station, a simple power tapping circuit, shown in Figure 15, was inserted into the current loop. An LED was placed in the current loop, introducing a voltage drop of 2.1 V. The voltage budget of the current loop allowed this reduction. An LC filter was placed across the LED to ensure switching noise from the DCDC conversion would not distort the current loop communication. An energy harvesting boost converter was placed across the LED to use the tapped current to charge a 0.47 F super capacitor. Here ATEX-II B T6 intrinsic safety requirements necessitated clamping with a $3.5 \mathrm{~V}$ shunt voltage limiter, and a series 4.7 $\Omega$ current limiter to prevent explosion due to super capacitor overvoltage, or sparking due to current surges. A second DCDC converter maintained a stable voltage for the base station electronics while the super capacitor voltage varied. The high power RF transceiver and amplifier was powered from the second converter, while the microcontroller was powered from the first. This allowed the radio electronics to be fully deactivated in the sleep state. Therefore the sleeping current consumption, as a parasitic draw on the energy harvester, was limited to $41 \mathrm{uA}$, including the microcontroller sleeping current (300 $\mathrm{nA})$, quiescent current of one active converter $(24 \mathrm{uA})$ and one shutdown converter $(10 \mathrm{uA})$ in addition to $7 \mathrm{uA}$ leakage current from the $0.47 \mathrm{~F}$ super capacitor.

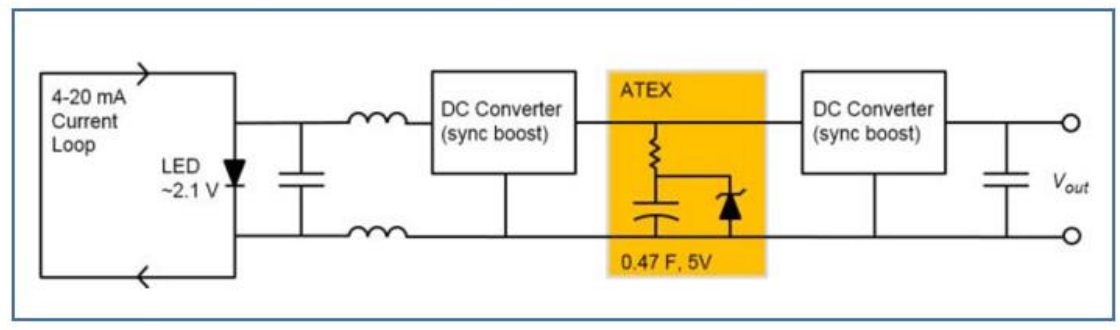

Figure 15. Industrial current loop energy harvester for base station power autonomy including current loop inserted LED, filtering circuit, DC converter charging super capacitor with series current limiting resister, and shunt diode overvoltage detector, second DC converter and output voltage to base station.

\subsubsection{Test and Evaluation}

Each of the developed technologies were tested in a laboratory environment before system integration and piloting tests at Metso Flow Control Oy, presented in Figure 10. The tests and results are here summarised and described in more detail in Publication III. The system was demonstrated to peers in the field, summarised in [4] and available as an online video [91].

The MEMS acoustic emission sensor of Publication III was tested and benchmarked against a commercial piezoelectric device; the PACR15-alpha from Physical Acoustic Co. The test setup is illustrated in Figure 16a. An industrial processing valve, with compressed air used to generate flow was used for the test. A deliberate defect was applied to the valve in the form of a small cut-out at the valve interface in order to generate turbulent flow and related acoustic emissions. The flow through the cut-out, and therefore the acoustic emission, was controlled by regulating the pressure across the valve and the flow was 
measured on the opposite side. Both the MEMS and piezoelectric sensor were read out with the same front end and RMS detector allowing a direct comparison of the sensor itself.

The sensors were compared for leakage flow rates from o to $600 \mathrm{ml} / \mathrm{min}$ and the results of the comparison are shown in Figure 16b. The correlation between the sensors is clearly observed from the results. The threshold for leakage detection was approximately $140 \mathrm{ml} / \mathrm{min}$, and the MEMS sensor presented approximately $5 \%$ greater RMS voltage output for leakage rates below 300 $\mathrm{ml} / \mathrm{min}$ and approximately $10 \%$ for leakage rates above $300 \mathrm{ml} / \mathrm{min}$. Additionally, both sensors displayed non-linear behaviour at $400 \mathrm{ml} / \mathrm{min}$, but the MEMS sensor exhibited a less severe nonlinear effect. From this test, it can be concluded that the MEMS sensor functioned well and presented some improvements over the commercial piezoelectric device.

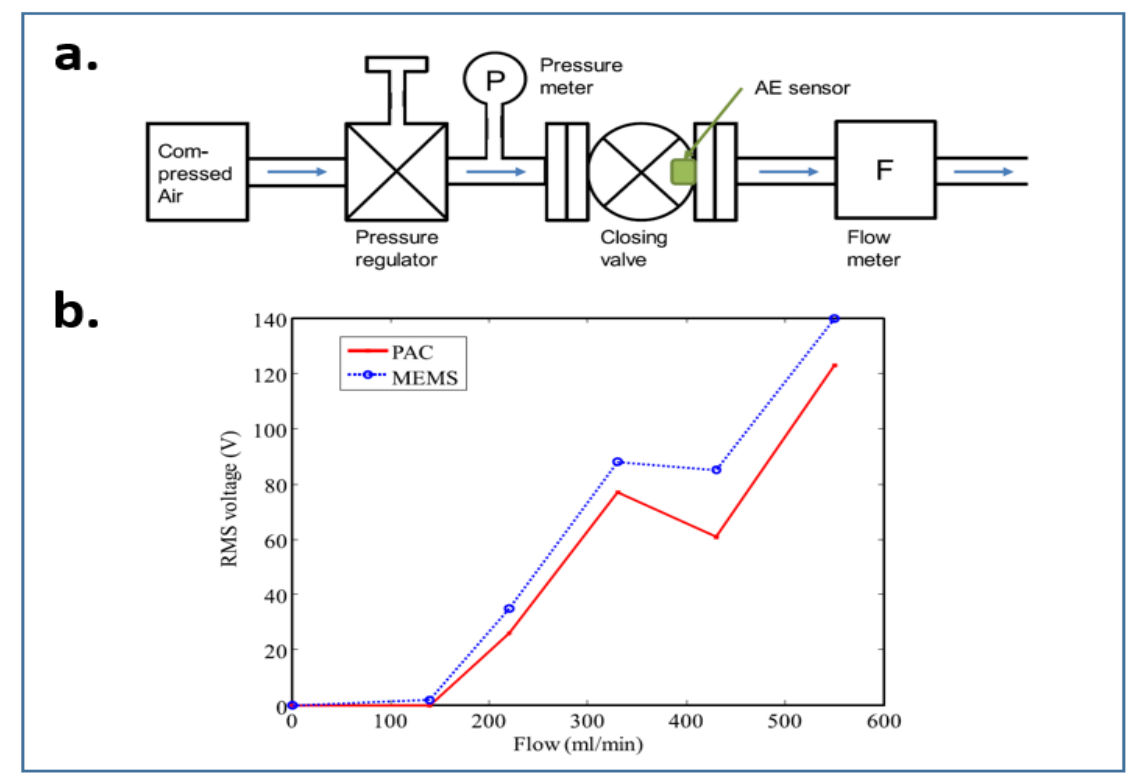

Figure 16. Comparison of MEMS acoustic emission sensor with commercial device. (a) Diagram of the test setup showing compressed air source, valve pressure regulator and meter, closing valve with etched leakage defeat, placement of the acoustic emission sensors, and output flow (leakage) meter. (b) RMS voltage detected vs leakage flow for leakage rates of 0 to 600 $\mathrm{ml} / \mathrm{min}$.

The radio system was tested to determine the reliability of the developed wake-up radio. For the tests a 24-bit packet was transmitted including the 16bit wake-up preamble and 8-bit wake-up address. The system was evaluated in terms of wake-up success, corresponding to successful detection of the whole 24-bit wake-up packet, with increasing distances between the base station and the sensor node. Two sensors, with only one-bit difference in their addresses, were placed at equal distances to test also the system's robustness to false wakeup. The system was evaluated where 100 wake-up attempts were made at each 
distance, and the packet error rate (PER) was calculated as the percentage of successful wake-ups. The tests were carried out at distances above $5 \mathrm{~m}$, at steps of $1 \mathrm{~m}$ up until $10 \mathrm{~m}$ and $0.5 \mathrm{~m}$ steps above $10 \mathrm{~m}$. Test were conducted both indoors in a long corridor and outdoors in effective free space, with no obstruction within $3 \mathrm{~m}$ or 25 wavelengths from the transmitter or receiver. In both cases the transmitter and receiver were placed approximately $1 \mathrm{~m}$ above the ground, and there was a direct line-of-sight between the transmitter and receiver. The distance was increased along this line-of sight between the transmitter and receiver.

The PER rate results for the evaluation are shown in Figure 17 where the measured results are compared with the theoretical PER calculated in a way as presented in ${ }^{[88]}$. In the indoor case the wake-up performed reliably up to a distance of $16 \mathrm{~m}$, with some exceptions between 11 and $14 \mathrm{~m}$, likely due to destructive interference of transmitted and reflected waves in the corridor. Above $17 \mathrm{~m}$ the PER increases sharply to $25 \%$ and already at $19 \mathrm{~m}$ the wake-up success reached zero. In the outdoor free space measurement, near zero PER was observed up to $20 \mathrm{~m}$ distance, with $95 \%$ wake-up success rate up until $21.5 \mathrm{~m}$. At $22 \mathrm{~m}$ the PER abruptly increased and above $23 \mathrm{~m}$ the wake-up success rate reached zero. In both cases the PER performance achieved longer distances than the theory predicted, and the transition from low to high error rates occurred much more abruptly than predicted. The differences are likely due to environmental effects such as propagation path reflections and presence of coherent interference signals. The targeted wake-up range of $10 \mathrm{~m}$ was significantly exceeded in each case.

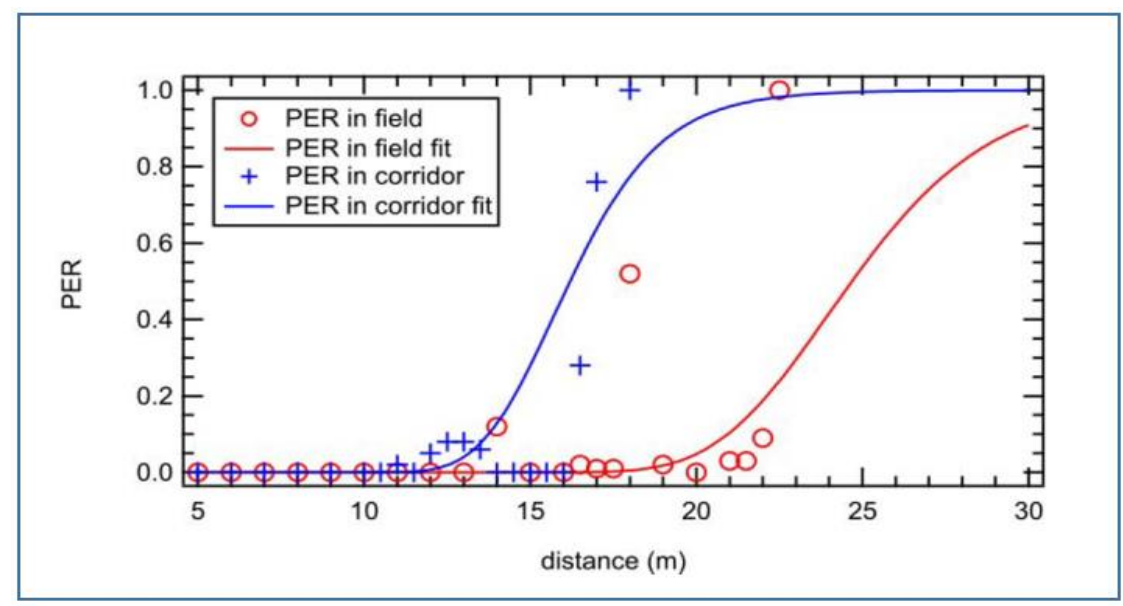

Figure 17. Range and reliability tests of wake up radio showing theoretical and measured package error rates of the wake-up radio tests, in an indoor corridor (blue) and outdoors (red).

The base station energy harvester was evaluated by measuring the tapped current and monitoring the super capacitor voltage through the harvesting and measurement cycle. The simple energy harvesting technique allowed $120 \mathrm{uA}$ $(250 \mathrm{uW})$ to be tapped in the minimum current loop drive condition of $4 \mathrm{~mA}$, 
and $600 \mathrm{uA}(1.26 \mathrm{~mW})$ in the maximum drive condition of $20 \mathrm{~mA}$. In the minimum loop drive state, the harvester needed 15 minutes to charge the super capacitor from o to $2.4 \mathrm{~V}$, a threshold above which measurements could be conducted. In the $4 \mathrm{~mA}$ drive state, enough energy was available to the energy harvester to perform one measurement cycle every 5 minutes, thereby allowing more than 256 sensor nodes to be interrogated over a 24 hour period.

The sensor node thermal energy harvester was evaluated by applying a heating coil to the bolt threads. The TEG element was encased in a plastic shell between the bolt threads and heat sink fins which allowed monitoring its temperature using a thermal infrared camera. The power harvesting circuit initiated and harvested $44 \mathrm{uW}$ at a temperature difference of $6 \mathrm{~K}$ across the TEG. As the temperature increased the harvested energy increased to $450 \mathrm{uW}$ at $10 \mathrm{~K}$ and $5 \mathrm{~mW}$ at $46 \mathrm{~K}$. The extracted energy of the harvester for temperature differences up to 46 $\mathrm{K}$ is illustrated in Figure 18 and compared with the maximum output power of the energy harvester. A difference of approximately $50 \%$ was observed between the realised power and maximum, due to losses in the power conversion. Still, the energy harvester was shown to generate enough power (>22 uW) to sustain the sensor node in sleep / listening mode at a temperature difference of $6 \mathrm{~K}$ with enough overhead to store power for measurements every 12 hours.

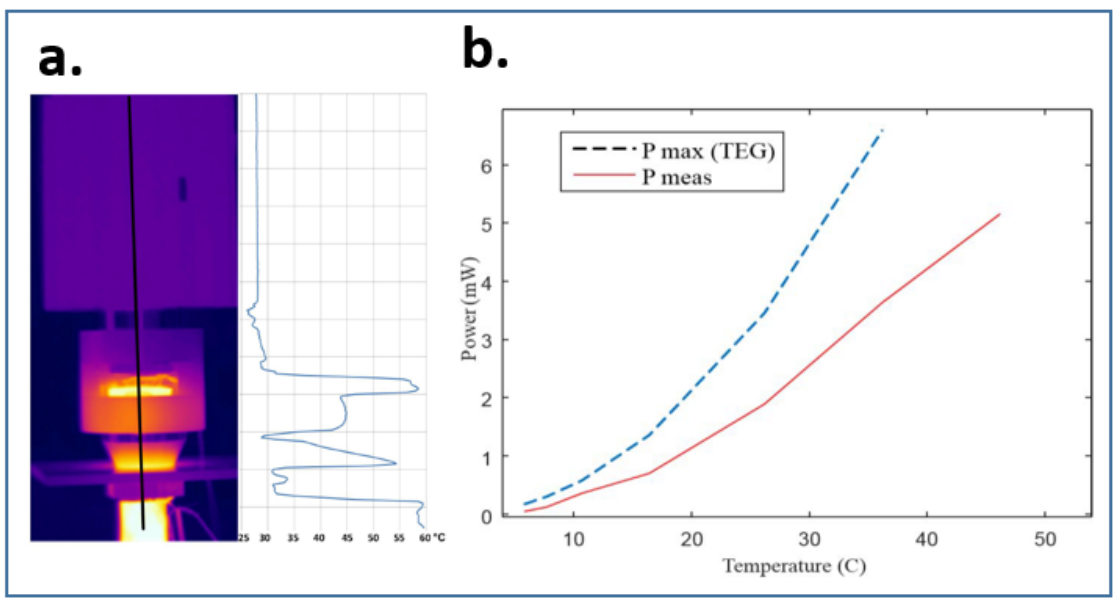

Figure 18. Thermal energy harvester power tests. (a) Example of thermal camera imaged energy harvesting bolt sensor. (b) Plot of harvested energy vs thermal camera imaged temperature difference across the TEG.

\subsubsection{Summary}

A wireless valve leakage detection system had been developed with energy autonomy achieved using harvesting circuits on both the sensor and base station sides. The device integrated a MEMS acoustic emission sensor which was benchmarked against a commercial piezoelectric device and shown to achieve similar and even slightly improved performance. A novel wake-up radio tech- 
nology was applied to allow asynchronous measurement to be performed between a base station device and up to 256 remote sensor nodes. The wake-up radio achieved effective operation at distances of $16 \mathrm{~m}$ (indoor corridor) and 21 $\mathrm{m}$ (outdoor in free space). The sensor nodes were made power autonomous with a thermal energy harvesting embodiment that extracted enough energy to perform a measurement every 12 hours with a $6 \mathrm{~K}$ temperature differential across the TEG element. The base station device was made power autonomous using an industrial current loop energy harvester which extracted enough energy, in the lowest loop drive state to perform more than the required 256 measurements in a 24 hour period. The system was delivered and piloted at Metso Flow Control Oy. 



\subsection{Wirelessly Powered Soft Robot}

The advancement of micro-robotics is greatly limited by the need for motors to drive mechanical joints and a power source. Batteries can be used but they add severely to the size weight and cost of the device. The work of this thesis develops a soft caterpillar like robot which uses shape memory actuators (SMAs) to remove motors and mechanical joints and motorised actuators. An inductively coupled wireless powering technique is developed to remove the need for a power source. The thesis delivers, to the author's knowledge, the world's first wirelessly powered soft robot. This early stage research focuses on the wireless actuation of the robot and sensors can be added to the platform in the future.

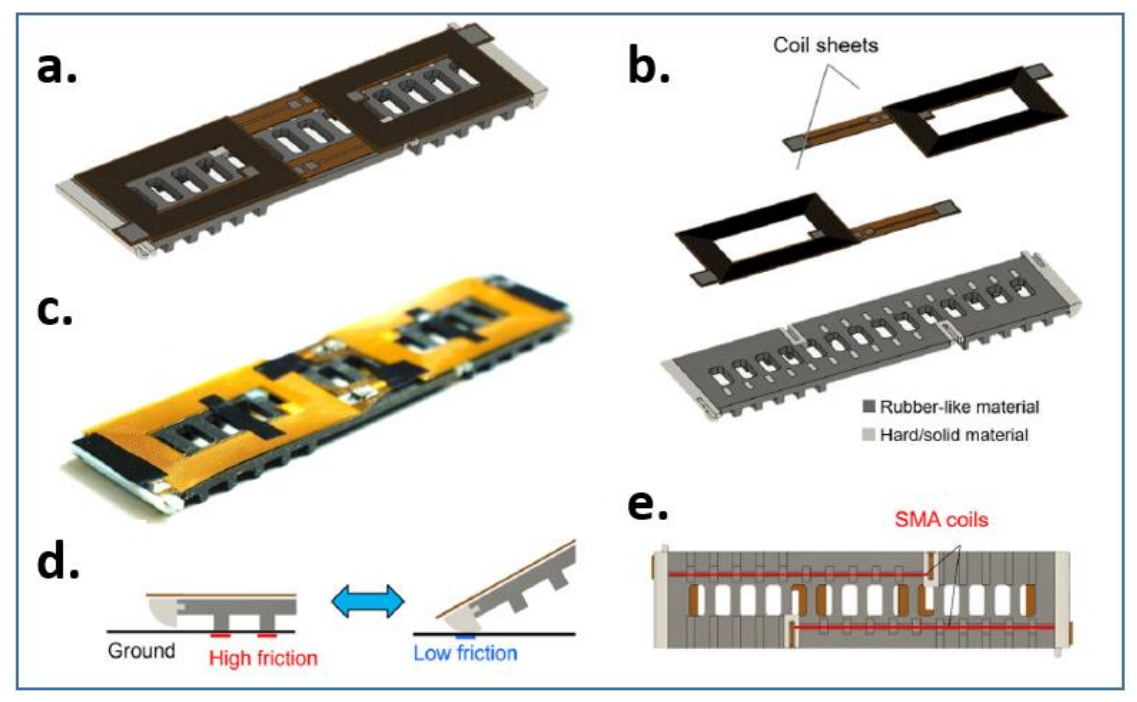

Figure 19. Illustration of the wirelessly powered soft robotic caterpillar. (a) Model of the 3D printed caterpillar with assembled receiver coils. (b) Exploded 3D model showing two receiver coils above the 3D printed caterpillar. (c) Photograph of the 3D printed device with receiver coils assembled. (d) Illustration of the high and low friction states which enabled caterpillar locomotion. (e) 3D model view from underneath, showing the assembly of the SMAs.

The soft robotic system is illustrated in Figure 19. A 3D model of the soft robotic caterpillar is shown in Figure 19a. Exploding the model, Figure 19b shows the assembly of two coils which were used as wireless power receivers for two SMA actuators. The robot was 3D printed using an Object26o Connex3 3D printed device. A rubber-like material (TangoBlack) was used for the soft body, shown in black in Figure 19b and a hard/solid material (VeroWhite+) was used for the hard legs, shown in white in Figure 19b. The coil sheets are fabricated on a polyimide flexible substrate with 18 um etched copper. A photograph of the soft robot with assembled receiver coils is shown in Figure 19c. The coils were attached on the top side of the robot, while two SMA actuators were assembled through the body as shown in Figure 19d. Actuation of the robot was achieved 
by controlling the current in the SMA actuators to generate contraction, in combination with the alternating friction condition between the smooth hard white ends of the structure and the high friction rubber legs as shown in Figure 19e. Further details of the locomotion are provided in Publication IV. This work is a direct development of ${ }^{[58]}$, where the soft robotic caterpillar with wired powering was developed.

\subsubsection{Requirements and Constraints}

The developed wireless power transfer system was required to independently control the currents in two SMA actuators in order to generate locomotion, in a manner similar to that proposed for the wired device in ${ }^{[58]}$. 200 to $300 \mathrm{~mA}$ of current needed to be induced in each SMA to generate enough heat and therefore contraction force for the robot locomotion. The dimensions of the robot, $100 \mathrm{~mm} \times 25 \mathrm{~mm}$, restricted the receiver coil shapes and sizes. The coils had to be designed to be large enough to receive the needed power, but small and separated enough not to strongly couple to one another. Due to the flexible nature of the robot, the coils themselves would need to exhibit equal, or greater flexibility that the robot body so as not to deteriorate the spring constant of the whole device. A target of $20 \mathrm{~mm}$ standoff was set for the wireless power transfer system, to allow actuation through a standard table, for example. The range of locomotion for the proof of concept, was restricted to a circle of $150 \mathrm{~mm}$ diameter. The main design goal was to maximise the power transfer efficiency between the transmitter coil and each SMA actuator.

\subsubsection{System Level Design}

The system level description of caterpillar and transmitter loop is provided in Figure 20. The signals for stimulation of the SMA receivers were generated by $\mathrm{s}$ R\&S SMBV100A Vector Signal Generator and amplified by a 75 W E\&I A-075 Class-A Linear Amplifier. The transmitter loop (shown in blue in Figure 20) was implemented using litz copper wire coil sandwiched between two polyurethane sheets. The robotic caterpillar itself (shown in green in Figure 20) comprised of the two SMA actuators, which were manifested electrically as largely resistive loads, coupled to two resonant coil receivers. Power was inductively coupled between the transmitter loop and the receiver coils inducing currents in the SMA actuators. The resonance frequency of each receiver coil was tuned at different frequencies so that the SMA actuators could be independently controlled, as required to generate a wave of contraction through the caterpillar to provide locomotion. 


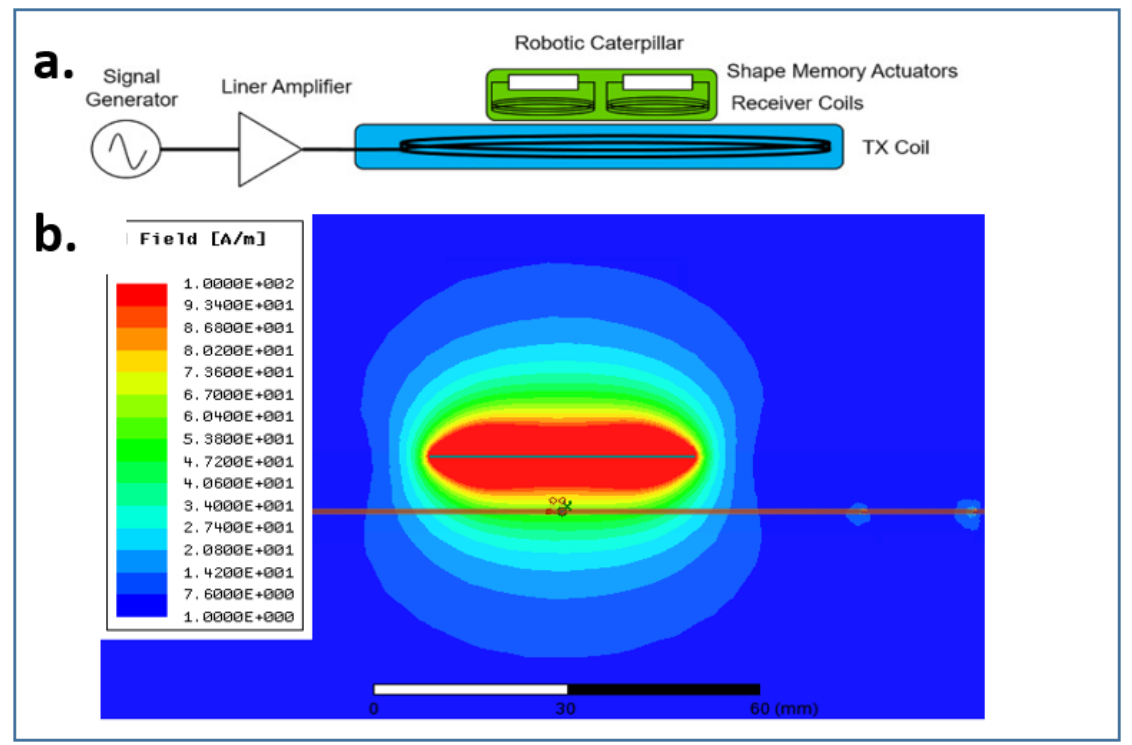

Figure 20. Block diagram and magnetic coupling simulations, (a) Block diagram showing the soft robotic caterpillar with two SMA actuators coupled to WPT receiver coils. Transmitter is driven by a linear amplifier controlled by an RF signal generator. (b) High frequency structure simulator (HFSS) simulation, showing the magnetic coupling between one SMA loaded receiver coil, and the transmitter, at a standoff of $10 \mathrm{~mm}$.

\subsubsection{Wireless Power Transfer}

The WPT system is based on near field resonant inductive coupling between the transmission loop and the receiver coils. A centre frequency was chosen as 8.5 $\mathrm{MHz}$, as a compromise between coupling distance achievable between large and small coils, resistive losses in the coils and avoidance of the coils self-resonant frequencies. This frequency also allows the design of receiver coils in the dimension order of the caterpillar, and a transmitter coil of size enough to demonstrate the caterpillar's locomotion. The SMA actuators were tuned to 8.25 $\mathrm{MHz}$ and $8.75 \mathrm{MHz}$ and a frequency sweeping input signal was used to alternately stimulate the coils.

A circuit representation of the WPT system is illustrated in Figure 21a. The signal generator and power amplifier is represented as an ideal voltage source with $50 \Omega$ source resistance. The transmitter coil is represented as an ideal inductor and parasitic equivalent series resistance. The parasitic parallel capacitance of the coil is omitted, as it is designed such that its effect is minimal at the transmission frequency. The transmitter loop is tuned to series resonance at the centre frequency of 8.5 MHz with capacitor $C 1$. Each receiver coil is represented as an ideal inductor and the coils equivalent series resistance, again parasitic parallel capacitance is omitted. The coils are tuned to series resonance targeted at 8.25 MHz and 8.75 MHz with capacitors $C_{2}$ and $C_{3}$. The SMA actuators are represented by $25 \Omega$ resistors, with a series inductance of $145 \mathrm{nH}$. The coupling between the transmitter loop and receiver coils is represented by the coupling coefficients $K 1$ and $K 2$, with a parasitic coupling between the two receiver coils 
of $K 12$ which is minimized by applying a separation of $10 \mathrm{~mm}$ between coil edges.

The circuit shown in Figure 21a (including parallel parasitic capacitances, and SMA inductance) was simulated in LTSPICE. Values for the circuit elements, detailed in Publication IV, represent those measured from the fabricated coils while the coupling factors were estimated based on HFSS simulations. The source frequency was swept from 6.5 - 10.5 MHz and the current induced in each SMA $\left(R_{4}\right.$ and $\left.R_{6}\right)$ is plotted in Figure $21 \mathrm{~b}$. A maximum current of $220 \mathrm{~mA}$ is induced in each SMA actuator at frequencies of 8.2 and $9 \mathrm{MHz}$ respectively. Some frequency deviation is observed due to the over coupling of the receiver coils to the transmitter loop. The effect of parasitic coupling between coils is also demonstrated from the simulation where each coil exhibits small current peaks of $75 \mathrm{~mA}$ at 7.8 and 9.3 $\mathrm{MHz}$ respectively. The induced $75 \mathrm{~mA}$ was not enough to generate an actuation response in the SMAs so it was considered tolerable.

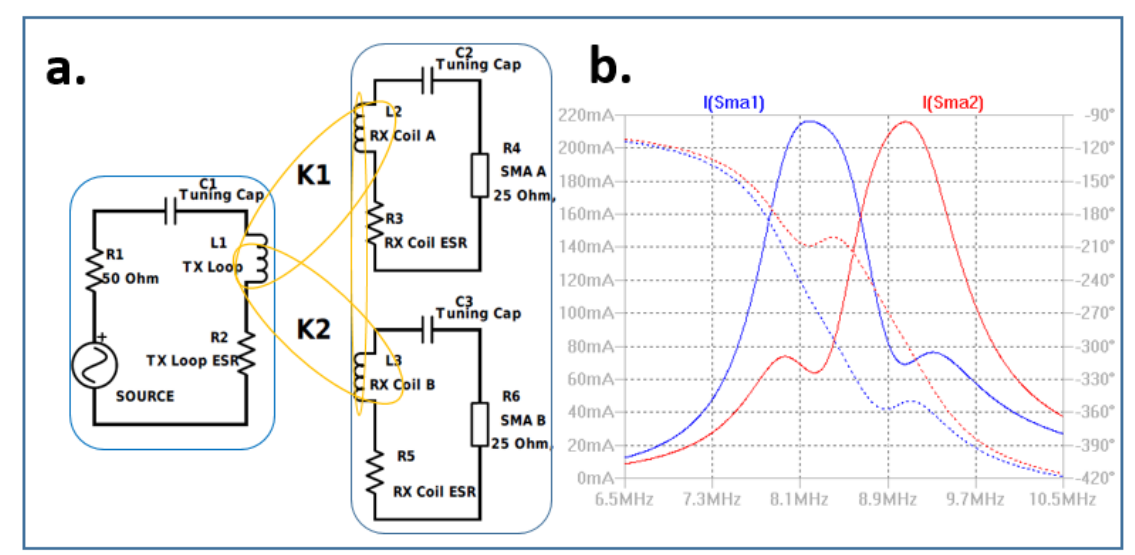

Figure 21. Electrical schematic and simulation of WPT system. (a) Electrical model of the transmitter (tuned by $C_{1}$ to resonance at $8.58 \mathrm{MHz}$ ) and two receiver coils tuned to resonance at 8.31 and $8.71 \mathrm{MHz}$ with $C_{2}$ and $C_{3}$ and coupled to SMA actuators. Coupling to the transmitter given by $K_{1}$ and $K_{2}$, and inter-receiver coil coupling of $K_{12}$. (b) LTSPICE simulation of the current coupled to the SMAs for a $20 \mathrm{~V}$ source and coupling coefficients of $K_{1}, K 2=0.1$ and $K_{12}=0.01$.

In order to approximate the coupling coefficient between the transmitter loop and each receiver coil as constant, the key design goal of the transmitter coil was the generation of a uniform magnetic field over the diameter of the loop. To that end, the design methodology of the transmitter loop was based on an achievement of uniform magnetic field in a planar loop found in the literature [92]. With reference to the published design, a 5 turn loop of $150 \mathrm{~mm}$ diameter was designed. HFSS simulations, based on a solid copper loop wire of $1 \mathrm{~mm}$ diameter, between two $4 \mathrm{~mm}$ thick polyethylene sheets estimated an impedance of $2+$ j365 (inductance of $6.8 \mathrm{uH}$ ) with a Q-factor of 183 and self-resonant frequency (SRF) of 28.6 MHz. Simulations showed a magnetic field uniformity of $81 \%$ within the diameter of the loop. The loop was fabricated from linz copper wire (660/46) between two $4 \mathrm{~mm}$ polyethylene sheets. The impedance of the real 
loop was measured as $3.1+\mathrm{j} 353$ (inductance of $6.3 \mathrm{uH}$ ) and Q-factor of 114 . The differences between simulation and measurements can be attributed to additional losses in the copper wire assumed perfect in simulation, and underestimation of the dielectric loss constant of the polyethylene. The fabricated loop was tuned to series resonance with a capacitor of $51.5 \mathrm{pF}$. Further details of the coil design methodology, simulations and measurements are provided in Publication IV.

The design goals of the receiver coils were to extract maximum power from the transmitter's magnetic field, to provide maximum power to the SMA load, and to minimise cross coupling between the two receivers. To maximise the coupling, the target was to design a coil with the largest possible surface area, and self-inductance. The power received by the coils would be divided between loss in the coils' series resistance and the SMA load. Therefore the coil design was optimised in terms of its Q-factor at 8.5 MHz. Etched copper of $18 \mathrm{um}$ thickness, on 38 um polyimide was selected as the fabrication process. Keeping the coil area fixed at $25 \mathrm{~mm} \times 40 \mathrm{~mm}$, an optimum was found, by HFSS simulation, in terms of the conductor width (100 - $1000 \mathrm{um})$ and the number of turns (3 19). An optimum conductor width was found to be 200 um and optimum number of turns to be 17. The simulation result was verified by the test of fabricated coils. Details of the optimisation simulations and comparison to the test of fabricated coils are provided in Publication IV.

\subsubsection{Test and Evaluation}

To evaluate the WPT system, the mutual coupling was measured for strongly and weakly coupled coils, where strongly coupled corresponds to the receiver placed on the polyethylene sheet ( $4 \mathrm{~mm}$ from transmitter coil) and weakly coupled corresponds to $20 \mathrm{~mm}$ standoff from the polyethylene sheet $(24 \mathrm{~mm}$ from transmitter coil). Coupling coefficients of 0.15 (strongly coupled) and 0.09 (weakly coupled) were measured. All of the following tests were performed with three identical, 17 turn, 200 um track width, receiver coils matched to series resonance at $8.31,8.58$ and $8.71 \mathrm{MHz}$ while the transmitter loop was matched to series resonance at $8.58 \mathrm{MHz}$. The power transfer efficiency, calculated as the square of the S21 parameter, of the system was evaluated using an R\&S VNA (Vector Network Analyser). The results, shown in Figure 22, demonstrate efficiencies for a $50 \Omega$ VNA load of 45 and $51 \%$, which map to 34 and $37 \%$ for 25 $\Omega$ SMA load, for receiver coils matched to 8.31 and 8.71. MHz. The modest efficiency values come from the small size of the receiver coils, the fact that they receive power at their own resonant frequency which deviates from the transmitter coils resonant frequency, and the non-optimal load matching of the whole WPT system. 


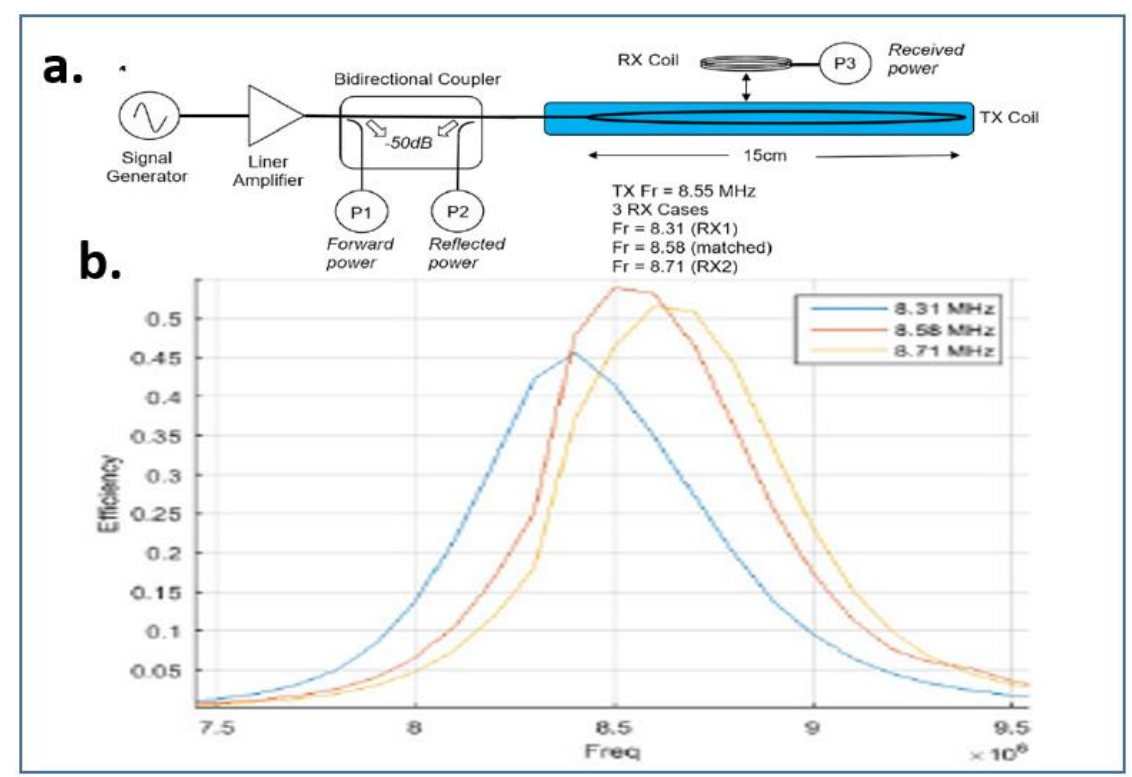

Figure 22. Power transfer efficiency measurements. (a) Setup for measurement of transmitted, reflected and received power. (b) Efficiency (calculated as received/transmitted power) for receiver coils at a standoff of $20 \mathrm{~mm}$, at resonant frequencies of $8.31,8.58$ and $8.71 \mathrm{MHz}$, with transmitter resonant at $8.58 \mathrm{MHz}$.

To compare with the power transfer and efficiency measurements, a test was devised to measure the real force generated in the SMA from the transmitters coil magnetic field. A single receiver coil was placed in the centre of the loop diameter at a standoff distance of $20 \mathrm{~mm}$, repeated for the coils resonant at 8.31, 8.58 and 8.71 MHz. The SMA (without the robot body) was loaded onto the coils as shown in Figure 19a, and one side was fixed to a rigid frame and the other to a Shimpo FGP-0.5 force measurement device. The frequency was incremented from $8-9.6 \mathrm{MHz}$ at intervals of $5 \mathrm{~s}$. The varying resonance behaviour of each receiver coil in delivering power to its SMA load was clearly observed in Figure 23. Maximum SMA forces of $41 \mathrm{~g}$ at $8.4 \mathrm{MHz}$ in the $8.31 \mathrm{MHz}$ matched receiver, $46 \mathrm{~g}$ at $8.6 \mathrm{MHz}$ in the $8.58 \mathrm{MHz}$ matched receiver, and $51 \mathrm{~g}$ at $8.8 \mathrm{MHz}$ for the 8.71 MHz matched receiver. Considering the below and above centre frequency matched coils, it was clearly observed that the force generated by their respective SMAs could be independently controlled by controlling the frequency of transmitted power and that a controlled wave of contraction could be stimulated in the soft robotic caterpillar by alternately actuating the SMAs by frequency sweeping the power source. 


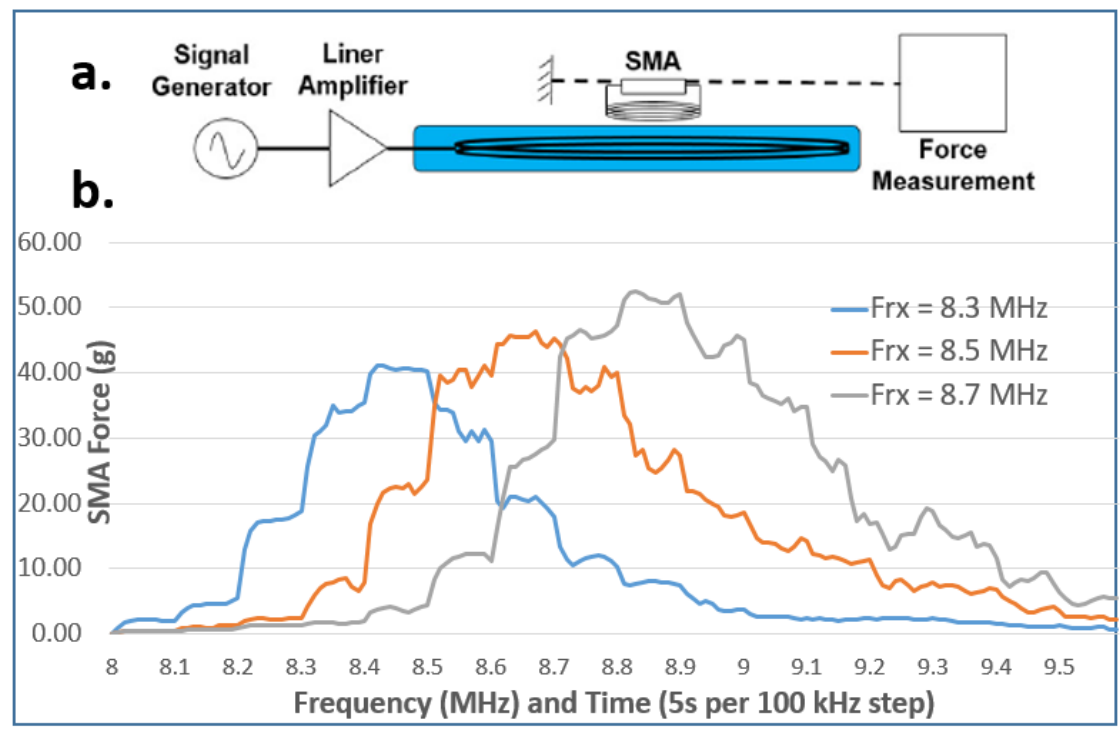

Figure 23. SMA force generation measurements. (c) Setup for the measurement of wirelessly stimulated SMA force. (b) Measured force generated in receiver coils at a standoff of $20 \mathrm{~mm}$, at resonant frequencies of $8.3,8.5$ and $8.7 \mathrm{MHz}$, with transmitter resonant at $8.58 \mathrm{MHz}$.

The power receiver coils, exactly as tested above, were assembled onto the caterpillar in a manner as shown in Figure 19. The caterpillar was placed above the transmitter coil, in a manner shown in Figure 20a, at a standoff of 20 mm, provided by a polystyrene separator. A gridded sheet of paper was placed under the caterpillar to easily measure its movements. The locomotion sweeps were synthesised in the signal generator outputting $2.5 \mathrm{~mW}$ and the frequency was incremented from 7.5 - 9.5 MHz, in steps of $100 \mathrm{kHz}$ with a dwell time of $100 \mathrm{~ms}$ between each step. There was a $1 \mathrm{~s}$ pause between sweeps to allow full cooling of both SMAs and therefore full relaxation of the caterpillar. One sequence of locomotion is summarised visually in Figure 24a. At the beginning of the sweep the power transferred to the SMAs is negligible and the caterpillar is in the rest state. As the sweep frequency reaches 8.3 MHz one SMA contracts fully. As the frequency reaches $8.5 \mathrm{MHz}$ both SMAs receive approximately the same power, and further increasing to $8.7 \mathrm{MHz}$ transfers the maximum contraction to the second SMA before both SMAs relax fully at the end of the cycle. This wave of contraction, in combination with the soft and elastic caterpillar body allowed the transfer of SMA contractive force to locomotion through the mechanism of alternating friction shown in Figure 19d. The direction of caterpillar movement can be alternated simply by changing the direction of the frequency sweep. The cycle described was repeated over a $28 \mathrm{~s}$ second period, and the forward locomotion of the caterpillar is plotted against time in Figure 24b. The caterpillar's forward movement occurs at approximately $7.5 \mathrm{~cm} / \mathrm{min}$. The locomotion tests of the device are more fully described in Publication IV. 

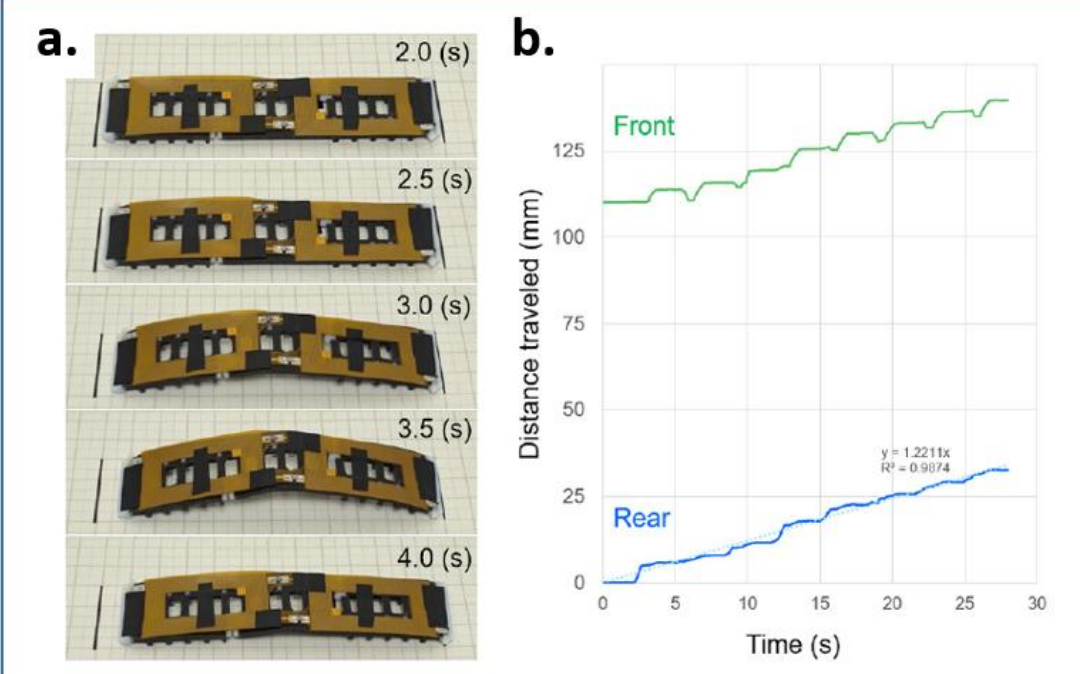

Figure 24. Demonstration of soft robotic caterpillar locomotion. (a) One locomotion cycle, where the transmission frequency is swept from $7.5 \mathrm{MHz}($ at $\mathrm{t}=0)$ to $9.5 \mathrm{MHz}($ at $\mathrm{t}=4 \mathrm{~s})$, in steps of $100 \mathrm{kHz}$ with $100 \mathrm{~ms}$ dwell time. (b) Measurement of caterpillar locomotion with time for $28 \mathrm{~s}$, for repeated frequency sweeps as described in (a).

\subsubsection{Summary}

This work has demonstrated a fully passive soft robotic caterpillar enabled by inductively coupled wireless power transfer. The device consisted of two SMA actuators coupled with two power receiver coils. A transmitter coil with an external diameter of $15 \mathrm{~cm}$ was simulated in HFSS and fabricated using linz copper wire. Independent control of the two SMAs was achieved by matching the receiver coils to series resonance at different frequencies $(8.31,8.71 \mathrm{MHz})$, the transmitter coil to series resonance at $8.58 \mathrm{MHz}$ and frequency sweeping the power source from 7.5 - 9.5 MHz. The power transfer efficiency of the system was evaluated and resulted in efficiencies of 34 and $37 \%$ for the 8.31 and 8.71 $\mathrm{MHz}$ matched coils. The system's capability to convert power received to SMA contraction force was observed to be $41 \mathrm{~g}$ and $51 \mathrm{~g}$ for the 8.31 and $8.71 \mathrm{MHz}$ matched coils. The locomotion of the device was analysed and found to occur at a rate of $7.5 \mathrm{~cm} / \mathrm{min}$. The fully passive soft robot achieves power autonomy, as the power and control signals are combined by sweeping the frequency of the wireless powering signal. 


\subsection{Passive Wireless Sensing}

The 'Zero Power Sensor System' has been under development at VTT for almost a decade. The work of this thesis is built on the direct background presented in $[59,77,93]$. The focus of the contribution in this work is the development and integration of the Zero Power Reader device introduced in Publication IX, along with its evaluation and steps taken to extend readout range, and increase the resolution of measurements detailed in Publication V. In addition, the integration of UHF RFID with the Zero Power Sensor for activation and identification is presented in Publication VIII.

The Zero Power readout mechanism is based on $3^{\text {rd }}$ order intermodulation. That is, the principle by which two incident electromagnetic signals are mixed when they encounter a non-linear element. This principle is exploited using a reader that transmits two frequencies, and a sensor tag which backscatters an intermodulation signal that depends on its measured parameter. The concept is illustrated in Figure 25, where the reader on the left transmits frequencies $f_{1}$ and $f_{2}$. The sensor node antenna (right of Figure 25) detects the incident signals, and mixes them in a diode to give a series of sum and difference frequencies. A choke inductor filters high frequency components, and only the low frequency difference $f_{2}-f_{1}$ is passed. A quartz resonator, selected with resonance around $f_{2}-f_{1}$ resonates the incident signal, and the maximum backscattered signal is achieved at that point where $f_{2}-f_{1}$ matches exactly the resonance of the quartz. The quartz oscillator is loaded by a sensor element which manifests its measured parameter in capacitance change (or in theory also inductance or resistance), that pulls the resonant frequency from its nominal value. The transmitted frequencies, $f_{1}$ and $f_{2}$, are swept so that their difference, $f_{2}-f_{1}$, covers the range of expected resonances of the oscillator-sensor combination and the loaded resonance frequency is determined by measuring the transmitted difference frequency at which the backscattered signal is at its maximum. The concept is described in much greater detail in Publication V and background to this work [59, 93].

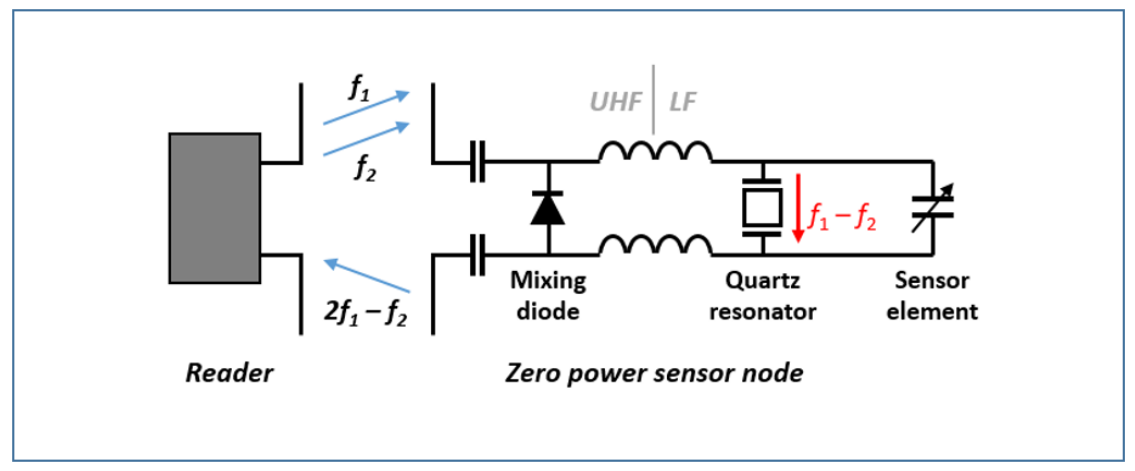

Figure 25. 3rd order intermodulation concept for sensing, reader device (left) transmitting two frequencies $f_{1}$ and $f_{2}$, Sensor node (right) with antenna and matching, non-linear mixing diode, RF choke inductor, quartz resonator resonant at $f_{1}-f_{2}$ and sensor element which pulls the quartz resonant frequency according to its measured parameter. 


\subsubsection{Requirements and Constraints}

The key limitations applied to the Zero Power System are borne of radio broadcast regulation constraints on usable electromagnetic spectrum and allowable transmit power. The maximum power over air at UHF RFID frequencies (around $868 \mathrm{MHz}$ ) is $2 \mathrm{~W}$ or $30 \mathrm{dBm}{ }^{[64]}$, which presents a limitation in the Zero Power System readout range, and highlights the requirement for power optimisation in the sensor tag and reader receiver front end.

In addition to the radio requirements, the zero power sensor tag needed to be as small and low cost as possible to compete with traditional battery powered sensors. The device needed to be designed to have a broadband matched antenna at $868 \mathrm{MHz}$. The frequency difference of the two transmitted signals had to be selected such that a high quality quartz oscillator could be found to resonance at that difference frequency. In addition, there are rather tight requirements on the sensor elements that can be combined with the zero power tags. They had to have low losses, corresponding to a high quality factor ( $>1000$ ) and manifest their measured parameter in terms of capacitance change in the region of $1 \mathrm{~s}-10 \mathrm{~s} \mathrm{pF}$.

The reader device itself needed to synthesise three required signals, at 865 $\mathrm{MHz}, 866 \mathrm{MHz}$ and $867 \mathrm{MHz}$, with an accuracy of $<10 \mathrm{~Hz}$ and be able to sweep them with a minimum achievable frequency resolution $<1 \mathrm{~Hz}$. The reader should combine the transmission signals and couple them to the air at the maximum permitted transmission power. The device needed to be able to detect sensor backscattered signals with a maximum achievable sensitivity and demodulate the sensor based intermodulation signal from them. It was desired that the reader would be compact and powered from a standard mains outlet, and that acquired data could easily be transferred to a cloud based IoT service.

\subsubsection{Radio Architecture}

While the $3^{\text {rd }}$ order intermodulation concept can work at any frequency, the limitations of available unregulated spectrum suggest open ISM bands at 433 $\mathrm{MHz}, 868 \mathrm{MHz}$ and $2.45 \mathrm{GHz} .868 \mathrm{MHz}$ was selected due to correlation with the UHF RFID frequency and allow the exploitation of a range of devices and components, such as power amplifiers, combiners, circulators, demodulators and antennae, optimised for use in RFID at the frequency. The UHF band allowed the design of compact antenna that was easily tuned and optimised to various sensor applications [59]. A UHF patch antenna was used for the reader giving a high gain and directivity to allow beam focused interrogation of sensor nodes.

The radio architecture of the Zero Power reader is illustrated in Figure 26. The architecture is divided into two parts; the transmitter shown in green which provides synthesis of transmission frequencies $f_{1}$ and $f_{2}$ and the superheterodyne receiver for demodulation shown in blue. The reader synthesises its two transmission frequencies using a high precision Voltage Controller Oscillator (VCO) 
coupled with a Phase Locked Loop (PLL), accurately controlled with an Analog Devices digital frequency synthesiser. The transmission frequency is isolated through a directional coupler, which also provides feedback for frequency correction within the PLL. The PLL output is band pass filtered to remove any harmonics before being output and amplified by a ZRL-3500+ power amplifier. The transmission signals, $\mathrm{f}_{1}$ and $\mathrm{f}_{2}$, are merged in a ZN2PD-920W-S+ combiner before being coupled to linearly polarised SPA 8090/75/8/o/V UHF patch antenna, through a Voyantic V2.o circulator.

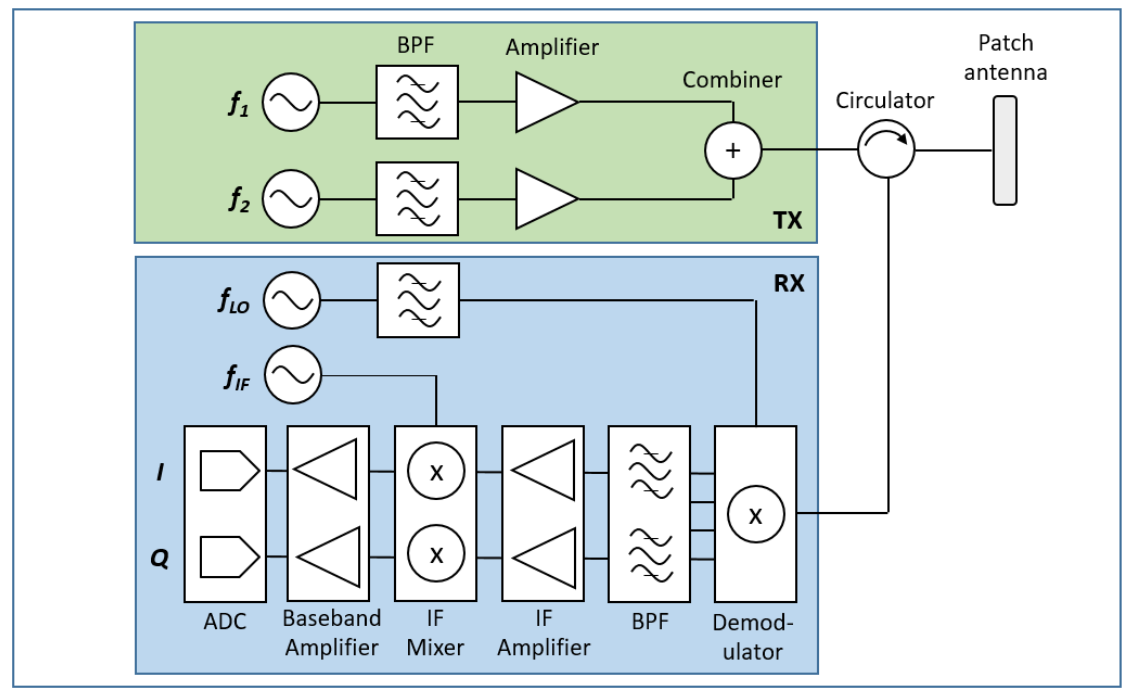

Figure 26. Radio architecture of the Zero Power reader. Transmitter (green) consists of $f_{1}$ and $f_{2}$ synthesisers, band pass filters, amplifiers and combiner. Receiver consists of centre frequency synthesizer, band pass filter, UHF demodulator, band pass filters, IF amplifier, low frequency mixer, baseband amplifier and ADCs giving $I$ and $Q$ channel outputs. External circulator is used to couple transmitted signals and received signals to the same antenna.

A third local oscillator frequency, $f_{\mathrm{LO}}$ for down conversion mixing, in the superheterodyne receiver is synthesised in a similar manor as far as the PLL output. The three generated frequencies are synchronised from a $40 \mathrm{MHz}$ reference oscillator, buffered in a low skew clock buffer and distributed to the frequency synthesisers. A $4^{\text {th }}$ frequency synthesizer is used in combination with the reference oscillator to generate synchronisation pulses that ensure frequency changes of the synthesisers are made exactly in sync. In the first implementation, $\mathrm{f}_{\mathrm{LO}}$ is the centre frequency, $\left(f_{1}+f_{2}\right) / 2$, and the frequency sweeping is accomplished by the sweeping of $f_{1}$ and $f_{2}$ in the opposite direction with respect to $f_{\mathrm{LO}}$.

The receiver acquires the backscattered single from the sensor node via the patch antenna through the circulator. The first stage of the receiver performs high frequency demodulation by multiplying the inputs $\left(\mathrm{o}^{0}\right.$ and phase shifted $90^{0}$ ) with the reference signal $f_{\mathrm{LO}}$. Here four signals $\left(I_{\mathrm{HI}}, I_{\mathrm{LO}}, Q_{\mathrm{HI}}, Q_{\mathrm{LO}}\right)$ are generated at the intermediate frequency (around $2 \mathrm{MHz}$ ). The IF signals are band pass filtered removing harmonics before being amplified through two-stage dif- 
ferential amplifiers. The intermediate frequency signals are further down converted in an analogue multiplier giving a baseband signal at the frequency sweeping rate (sub Hz). The baseband signal is amplified in a two-stage noninverting operation amplifier before being converted to digital signals in the microcontroller ADC. The radio architecture of the reader, both transmitter and receiver, is described in greater detail in Publication V.

The primary limitations of the Zero Power reader are highlighted and investigated in this work. Power optimisation, in respect of the zero power system indicates the maximisation of gains, minimisation of losses throughout the reader - sensor tag system. The optimisation leads directly to the extension of the readout range according to:

$$
r=\frac{\lambda}{4 \pi} \frac{\mathrm{P}_{\mathrm{tx}}{ }^{3 / 8}}{\mathrm{P}_{\mathrm{N}}{ }^{1 / 8}} G_{\mathrm{tx}}{ }^{1 / 2} G_{\mathrm{tag}}{ }^{1 / 2} S_{3}{ }^{1 / 8}
$$

Where $r$ is the readout range, $\lambda$ is the radio wavelength, $P_{\mathrm{tx}}$ is the transmission power, $P_{\mathrm{N}}$ is the reader noise level, $G_{\mathrm{tx}}$ is the transmitting antenna gain, $G_{\mathrm{tag}}$ is the sensor antenna gain and $S_{3}$ is the conversion loss from power received at the sensor to the IM3 produced. It is clear that the readout range is increased by increasing the wavelength, transmitted power, the antenna gains and the transmitted power, or by decreasing the conversion loss or reader noise level. Previous work ${ }^{[59]}$ optimised the sensor tag fixing the frequency, the conversion loss, and sensor antenna gain. On the reader side, the best available antenna is already in use, and the transmittable power is limited by regulation. Therefore, to extend the readout range, the reader noise level presents the only remaining optimisation parameter. The reader noise level is not defined by thermal noise, but rather the self-intermodulation within and between the reader components. $3^{\text {rd }}$ order intermodulation occurs not only at the sensor node mixing diode, but also at all non-linear elements of the reader where the two transmission frequencies are present. The most non-linear components of the reader are the power amplifiers, both within the frequency synthesisers and the output gain stage. The synthesised signals are too close in frequency for filtering, so the key design consideration is the isolation of the signal generation circuits from one another.

A second key limitation of the reader relates to the resolution of sensor readout. The change in the quartz resonance frequency can only be resolved with a resolution corresponding to the smallest frequency step possible in the intermediate frequency. The limitation is related to the minimum frequency step that can be made while maintaining synchronisation between the multiple synthesised frequencies.

A third limitation addressed in this work relates to the activation and identification of sensors. The readout mechanism described is inherently single channel, allowing only one sensor to be read out by the reader. 


\subsubsection{Reader Integration}

A block diagram of the integrated reader is provided in Figure 27a. The radio front end, described in 3.4.2, forms the core of the integrated reader. Power is provided to the reader through an $18 \mathrm{~V}$ DC adapter along with an on board DCDC converter which provides the range of positive and negative voltages needed for the system. The reader includes an ATMEL AVR ATXMEGA256A3BU microcontroller evaluation board (XMEGA-A3BU). As the 'brain' of the system, the AVR programs the synthesisers with the sweep parameters, initiates the sweep and synchronises the frequency steps with sync synthesiser. The microcontroller also acquires the I and Q signals, digitises them in its $\mathrm{ADC}$ and provides the data over USB to a PC user interface, or over Universal Asynchronous Receiver Transmitter (UART) to a Raspberry-PI3 which carries out communication of data to a cloud based service over RJ45 network cable or Wi-Fi. A cloud based monitoring service was implemented for data acquisition, visualisation and logging but it is outside the scope of this thesis.

For signal integrity and isolation, the front end was divided in to its UHF and baseband parts, implemented on separate Printed Circuit Boards (PCBs). The UHF PCB comprised the three frequency synthesizers and high frequency demodulator. Each of the synthesisers was shielded from one another, with separate LDO regulators inside the shield. All signals entering the shield were coupled in through a low pass LC feed through to minimise RF signal coupling. The reference clock generation and distribution was included on the UHF card, along with the generation of frequency step synchronisation pulses. The third shielded synthesiser included also the RF demodulator outputting the intermediate frequency signals to the baseband components implemented on a separate PCB. The baseband PCB, also shielded for isolation, included the IF amplifiers, DC mixers, baseband amplifiers and output near-DC I and Q signals, changing only at the frequency scan rate of the device, around 0.25 Hz.

A motherboard PCB was designed such that the four modules; UHF, baseband, AVR and Raspberry-PI could be mated vertically to it. The motherboard contained DCDC conversion circuits and provided power distribution to the modules on the board, each having their own LDO regulation. Digital signal routing was also done on the motherboard, while sensitive analogue and high frequency signals were connected using high quality shielded UFL cables. The large power amplifiers and combiner were assembled under the motherboard $\mathrm{PCB}$ and the circulator was placed externally to the reader. A $3 \mathrm{D}$ printed polymer casing was fabricated to house the reader. A system level block diagram of the reader is provided in Figure 27a and photograph of the integrated reader is provided in Figure 27b. A more detailed description of the reader architecture is presented in Publication V. 


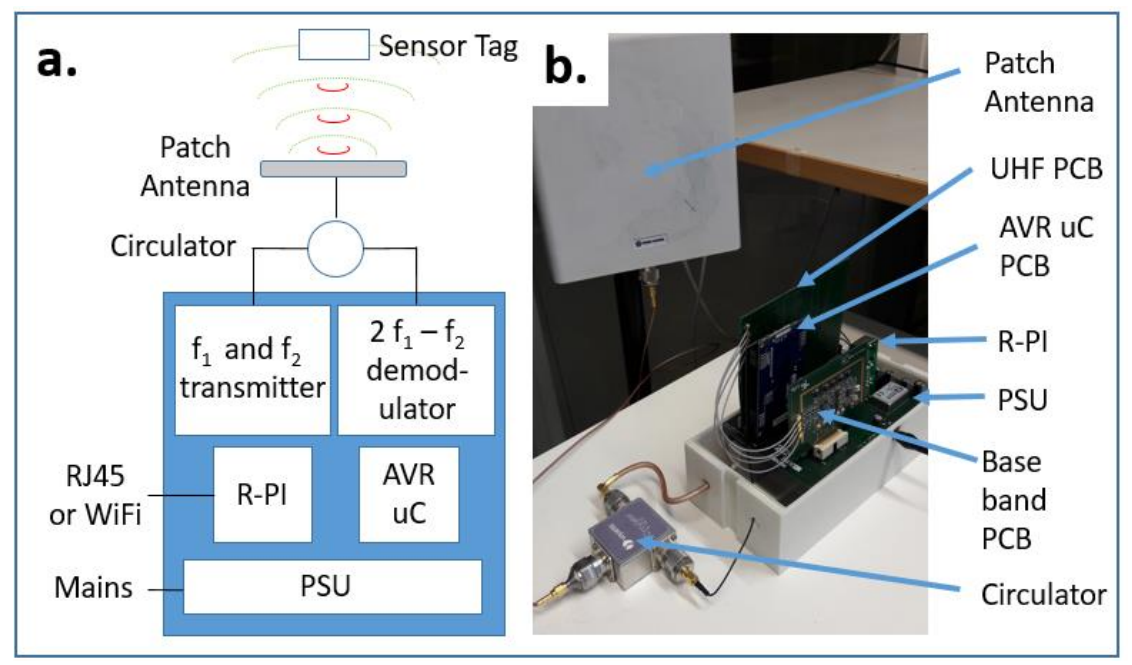

Figure 27. Zero power reader. (a) Block diagram of the reader comprising power supply, AVR microcontroller, Raspberry-Pi cloud interface, $f_{1}$ and $f_{2}$ synthesizer and transmitter, $2 f_{2}-f_{1}$ receiver and demodulator and external circulator and patch antenna showing transmitted (green) and sensor backscattered (red) signals. (b) Photo of the reader device as illustrated in (a).

\subsubsection{Frequency Sweeping}

In essence, the Zero Power reader investigates the resonant characteristics of a sensor node by scanning the range of frequency differences $f_{\mathrm{d}}=f_{2}-f_{1}$. The transmitted signals are swept over a range of $\pm \delta f$ to ensure that $f_{\mathrm{d}}$ sweeps over the loaded resonance frequency of the quartz. The tag mixes the difference frequency again with the incoming RF signals, and backscatters $2 f_{2}-f_{1}$ and $2 f_{1}-f_{2}$ to the reader. The backscattered signal is down conversion mixed with the centre frequency $f_{\mathrm{LO}}=\left(f_{2}+f_{1}\right) / 2$ so that the intermodulation frequencies are mixed down to the intermediate frequency $f_{\mathrm{IF}}=3 f_{\mathrm{D}} / 2 . f_{2}$ and $f_{1}$ are simultaneously swept in the opposite direction over a range of $\pm \delta f$, resulting in an $f_{\mathrm{d}}$ sweep of $\pm 2 \delta f$, and a sweep in the backscattered signals of $\pm 3 \delta f$. This is referred to as the Dual Side Band (DSB) approach, as shown in Figure 28a, and it allows the addition of both backscattered intermodulation signals $\left(2 f_{2}-f_{1}\right.$ and $\left.2 f_{1}-f_{2}\right)$ in the down conversion. However, this requires $f_{\text {LO }}$ to be swept over $\pm 3 / 2 \delta$. To maintain synchronisation in different frequency domains, with a common $40 \mathrm{MHz}$ quartz reference, a minimal step of $7.2 \mathrm{~Hz}$ in $f_{\mathrm{IF}}$ can be achieved, which correlates to a minimum step of $4.8 \mathrm{~Hz}$ in $f_{\mathrm{d}}$. This implies a limitation resolution of the sensor tags loaded resonance, translating to limitation of the resolution of the sensed parameter. The theory of the resolution is described in detail in Publication $\mathrm{V}$.

The frequency synthesiser (ADF4158) can achieve a minimal frequency step of $0.6 \mathrm{~Hz}$, while the AD5930 has a minimal increment of $2.4 \mathrm{~Hz}$. Therefore, to achieve maximum resolution, one has to devise a frequency mixing scheme, where the centre frequency is not swept. Keeping one frequency $\left(f_{1}\right)$ constant 
while sweeping the other $\left(f_{2}\right)$, to achieve the same frequency span on the tag side, $f_{\mathrm{d}}$ and thus $f_{2}$ must now be swept in the range of $\pm 2 \delta f$. Then, the backscattered signal sweeps over the range of $\pm 2 \delta f$ but in the opposite direction. So, if we set $f_{\mathrm{LO}}=2 f_{2}-f_{1}+f_{\mathrm{IF}}$, and sweep it over $\pm 2 \delta f$, then $f_{\mathrm{LO}}$ can be kept constant. The frequency spectrum of this technique is shown in Figure $28 \mathrm{~b}$ and for the other side band in Figure 28c. This case is referred to as Single Side Band (SSB), since only one single side band is used for down conversion and the other, sweeping at $\pm 4 \delta f$, is neglected. As a result, the received signal is expected to be $3 \mathrm{~dB}$ less than in the DSB scenario. Therefore, an 8 fold increase in resolution ( $0.6 \mathrm{~Hz}$ vs $4.8 \mathrm{~Hz}$ ) is achieved, at the cost of reduced reading range.

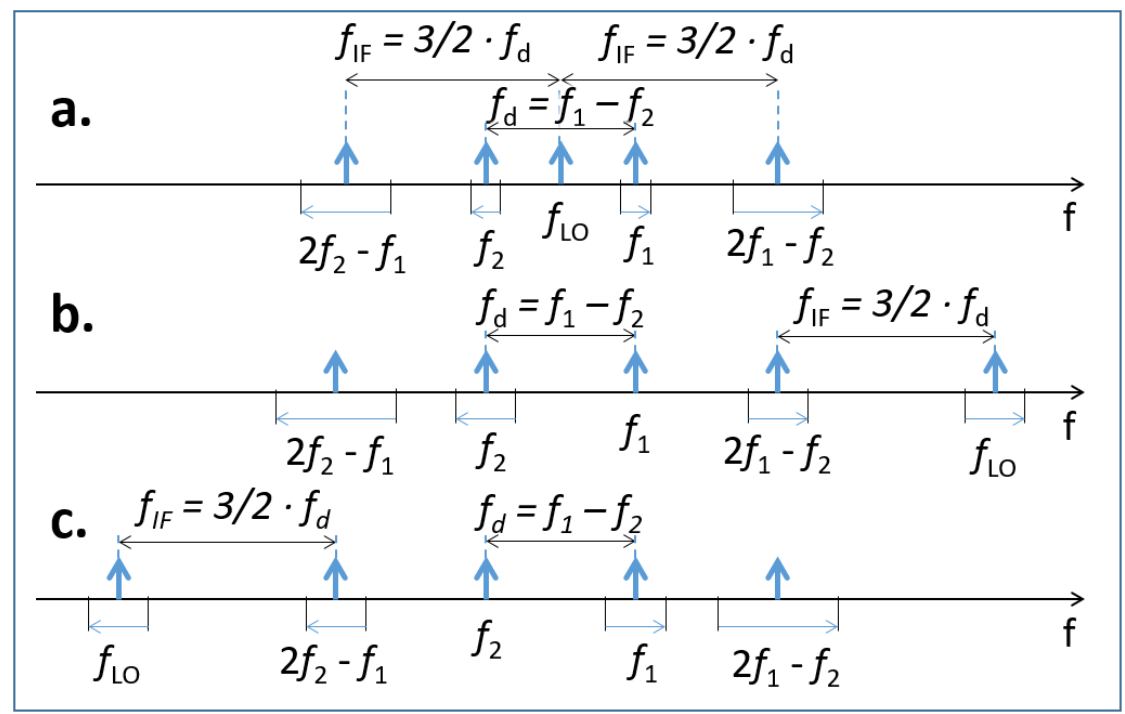

Figure 28. Frequency spectrum of the signals: a) DSB operation, both sidebands are used for maximal reading distance, b) and c) single sideband is used to achieve maximal resolution (at the cost of reduced reading distance). The sweeping ranges (horizontal blue arrows) are drawn proportional to one another, but are exaggerated in respect to distances.

\subsubsection{Activation and Identification}

The sensor readout mechanism is inherently single channel, which limits the practical deployment of the system. Some work has been done to investigate the frequency division multiple access (FDMA) of different sensors [94] but it is not considered a practical approach for a mass producible system. Instead, the combination of the Zero Power sensor with standardised UHF RFID technology was investigated as part of the work of this doctoral thesis.

The intermodulation is based on the non-linear characteristics of the diode element in the sensor. Therefore, to deactivate a particular sensor the diode needs to be biased out of its non-linear region of operation. To achieve this, the zero power sensor is combined with an NXP G2iL+ UHF RFID tag which includes a digital output. The output produces a voltage of $1.2 \mathrm{~V}$ which is enough to forward bias the diode into its linear region of operation. Using the RFID combination each sensor can be identified with the 96-bit electronic product 
code (EPC) address of the RFID tag, and activated/deactivated using the tag's digital output. The combination of the RFID tag with the zero power sensor is shown in Figure 29a. The digital output of the G2iL+ is connected across the quartz, so that it is allowed to apply a DC bias of $1.2 \mathrm{~V}$ to the diode through the filtering inductor. For the investigation of the concept a separate receiver antenna was designed for the sensor node and was placed at right angle to the ZPS antenna to minimise coupling between them. A circularly polarised transmit antenna was used in combination with a 'Feig Long Range Reader ID ISC.LRU100o' RFID reader for reading and writing the G2iL+ tags as shown in Figure 29b. Once the sensor is (de)activated, the RFID reader is shut down and the Zero Power reader's transmission illuminates the RFID tag such as to produce the digital output voltage to (de)activate the sensor node. Further details on the integration of UHF RFID with the ZPS is presented in Publication VIII.

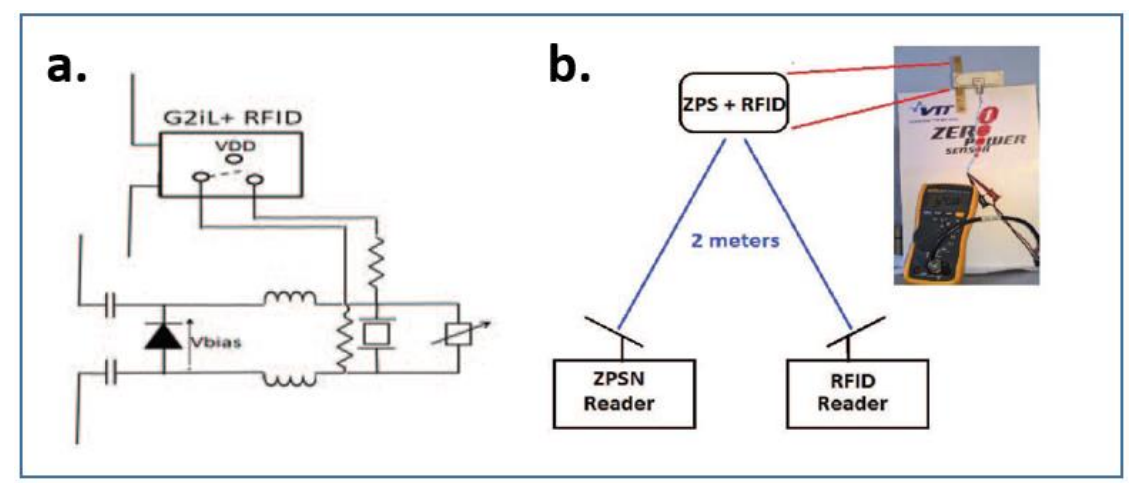

Figure 29. Activation and identification of Zero Power sensors using passive a G2iL+ UHF RFID tag. Showing the combination of the G2iL+ tag with the Zero Power Sensor (a) and the proof of concept test setup (b) showing the G2iL+ and ZPS tags with multimeter measurement of the digital output, and separate ZPSN and RFID readers.

\subsubsection{Test and Evaluation}

In addition to the externally imposed limitations on transmittable power, and with the optimisation of the sensor previously done [94], the remaining limitation of the readout range of the reader is the reader noise, based on its self-intermodulation. The range relates directly to the different transmission frequencies coupled back to the reader and the reader's IM3 products, a ratio defined as the spurious free dynamic range. Or more specifically, according the Eq. (1), the range depends on the ratio of $P_{\mathrm{tx}}{ }^{3} / P_{\mathrm{N}}$, where $P_{\mathrm{tx}}$ is the transmit power delivered to the antenna (measured at the circulator ANT terminal) and $P_{\mathrm{N}}$ is the self-intermodulation seen at the demodulator input (measured at the circulator $\mathrm{RX}$ terminal). The primary cause of the reader self-intermodulation was suspected to be the highly non-linear power amplifiers. Therefore, to fully understand their influence the system is examined with and without them. In particular, the coupling between the two power amplifier outputs through the combiner was an aspect of concern, so further investigation was conducted where additional 
isolation circulators were placed before the combiner, with the signals $f_{1}$ and $f_{2}$ input to their TX terminals, the circulator input connected to their ANT terminals, and the RX terminals terminated with $50 \Omega$. Therefore, the self-intermodulation evaluation of the reader was conducted under four scenarios, (a) where the power amplifiers are removed, (b) with the power amplifiers replaced, (c) with the power amplifiers removed and isolation circulators before the combiner and (d) with the power amplifiers replaced and isolation circulators before the combiner. The reader antenna was replaced with a $50 \Omega$ termination, and measurements were taken at three locations in the reader, with reference to Figure 26 (i) generated signal at the combiner output (circulator TX), (ii) transmitted signal to the antenna (circulator ANT) and (iii) received signal (circulator $\mathrm{RX).}$

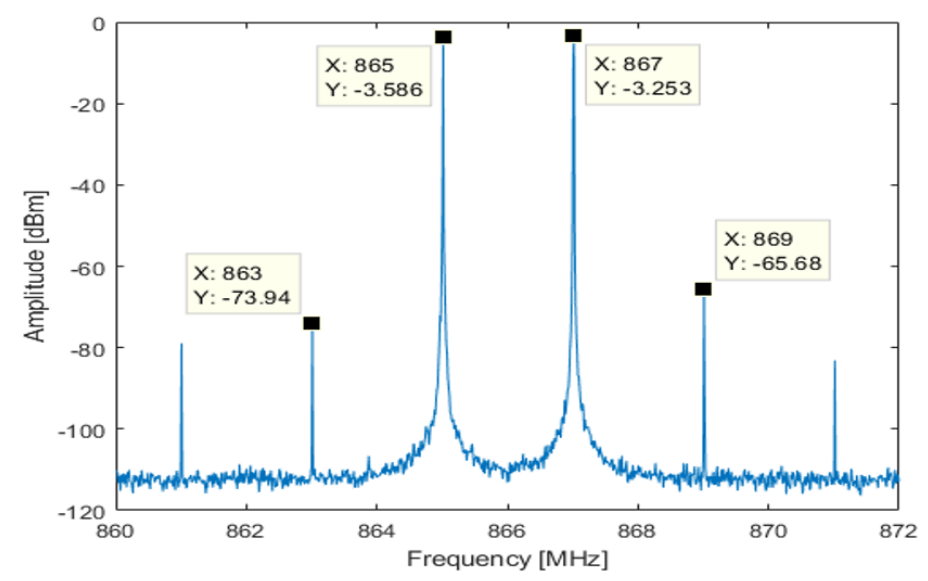

Figure 30. Evaluation of the reader's self-intermodulation. R\&S FSW $8 \mathrm{GHz}$ spectrum analyser measurement of the Reader \& Power Amps \& Isolation Circulators $(\mathrm{dBm})$ measured at the transmission circulator $\mathrm{RX}$ terminal. Transmission frequencies at 865 and $867 \mathrm{MHz}$, reader $3^{\text {rd }}$ order IM at 863 and $869 \mathrm{MHz}$, 5th order IM response at 861 and $871 \mathrm{MHz}$.

The reader's self-intermodulation was examined with an R\&S FWS 8 GHz spectrum analyser in the frequency range of $860-872 \mathrm{MHz}$. The results for case (d, iii) are illustrated in Figure 30. Here the reader including power amplifiers and isolation circulators are used and the power spectrum is measured at the transmission circulator RX terminal with a $50 \Omega$ termination in place of the antenna. The transmission frequencies at $f_{1}=865 \mathrm{MHz}$ and $f_{2}=867 \mathrm{MHz}$ are coupled back into the reader with respective powers of -3.6 and $-3.3 \mathrm{dBm}$. The powers at the IM3 frequencies $\left(2 f_{1}-f_{2}=863 \mathrm{MHz}\right.$, and $\left.2 f_{2}-f_{1}=869 \mathrm{MHz}\right)$ are respectively -73.9 and $-65.7 \mathrm{dBm}$. The SFDR is calculated as the average difference between the transmission frequency power and the IM3 power seen at the reader's demodulator input, and it's calculated to be $66.5 \mathrm{~dB}$ in this case. The $P_{\mathrm{tx}^{3}} / P_{\mathrm{N}}$ ratio is calculated between the IM3 products at the receiver and the transmit power delivered to the antenna, and is evaluated to be $126 \mathrm{~dB}$, comparing $P_{\mathrm{tx}}$ in (d, ii) and $P_{\mathrm{N}}$ at the IM3 frequencies in (d, iii). The isolation circulators, form the most significant design improvement in this work. Comparing 
the $P_{\mathrm{tx}} 3 / P_{\mathrm{N}}$ shows an increase of $24 \mathrm{~dB}$ when the isolation circulators are applied, and a corresponding extension in the read range can be expected as the following investigation will show. More details of all measurements taken are provided in Publication V.

The evaluation of the reader performance when interrogating one sensor was conducted using the reader hardware as described in Section 3.4.3, and a sensor comprising of the $2 \mathrm{MHz}$ crystal oscillator loaded with $1 \mathrm{pF}$, which is expected to pull the resonance frequency of slightly above $2 \mathrm{MHz}$. Both the SSB and DSB frequency sweeping approaches were investigated and both cases were measured with and without the isolation circulators in place. The tests were conducted in a corridor measuring $2 \mathrm{~m}$ width by $2.5 \mathrm{~m}$ height, and approximately $55 \mathrm{~m}$ long. The reader configuration was identical for all tests, including the radio cables and antenna. For the noise measurements, the transmission circulator was terminated with $50 \Omega$ in place of the antenna, to eliminate external disturbances in the noise measurements.

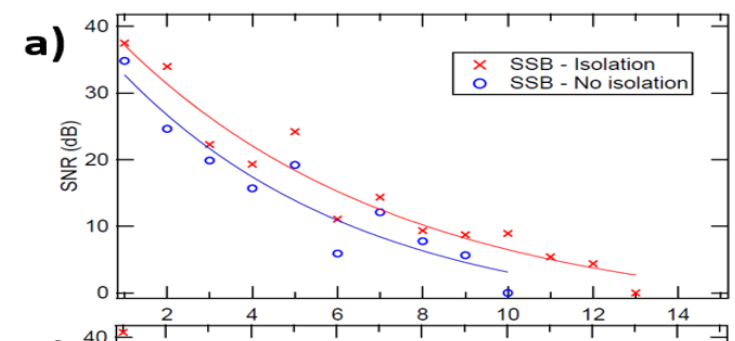

b)

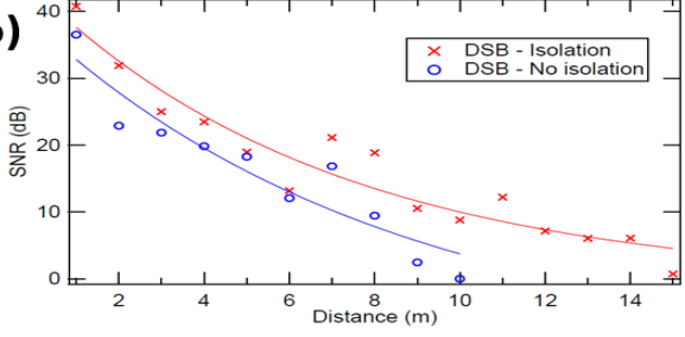

Figure 31. Evaluation results of the signal to noise ratio in $\mathrm{dB}$, for both the (a) SSB and (b) DSB readers, with (red) and without (blue) isolation circulators included after the power amplifiers and before the combiner in Fig. 27. Measured data points are shown along with trend lines.

The measurements, presented in Figure 31, were taken by analysing the baseband $I$ and $Q$ signals output from the receiver. The noise seen on the $I$ and $Q$ outputs includes both the thermal noise of the baseband and the more dominant reader self-intermodulation. The SNR was calculated as the ratio between the modules of the peak to peak $I$ and $Q$ signals divided by the module of the peak to peak noise on $I$ and $Q$ channels, and converted to $\mathrm{dB}$. The SNR with the SSB reader configuration, is shown in red; dotted for the case without isolation and solid including isolation. It is clear from this data that the isolation technique applied increases the SNR and extends the maximum readout range from $10 \mathrm{~m}$ 
to $13 \mathrm{~m}$. The DSB reader configuration is shown in blue, where again the dotted plot corresponds to non-isolation, and the solid plot includes isolation enhancement. Again, from this data we can clearly see that the isolation enhancement increases the SNR and extends the range to $15 \mathrm{~m}$, the longest readout range reported for the Zero Power Sensor system. Further analysis of the SNR and range results is included in Publication V.

The enhancement of the reader's resolution was evaluated by comparing the SSB and DSB frequency sweeping approaches as described in Section 3.4.4. The experiment was conducted under the same conditions as the SNR evaluation above, with the same sensor and a readout distance of $5 \mathrm{~m}$. The results, shown in Figure 32 represent the $I$ and $Q$ channel measurements, representing the backscattered and demodulated IM3 signal from the sensor, digitised in the reader's microcontroller ADC. The modulus signal is calculated as $\left(\sqrt{I^{2}+Q^{2}}\right)$. One single $I$ and $Q$ measurement is taken for each frequency step in the sweep. The DSB measurements, shown on the left, are taken with the minimum achievable frequency step of $4.8 \mathrm{~Hz}$. The limitation of the resolution is clearly observed, as the resonance peak is not well resolved in either the $I, Q$ or modulus signals. The SSB measurements shown on the right are also taken with the minimum achievable frequency step, $0.6 \mathrm{~Hz}$ in this case. Here the resonance peak is very well represented in the data, and even the high frequency noise can be resolved. While the quality factor of the sensor used in this test is low enough to be resolved by the DSB technique, it is clear that the peak resonance frequency can only be resolved with an accuracy of $+/-4.8 \mathrm{~Hz}$. In the SSB approach it can be resolved with $+/-0.6 \mathrm{~Hz}$. These can be mapped to a minimums capacitance resolution of $22 \mathrm{fF}$ in the DSB case and $3 \mathrm{fF}$ is the SSB case, for the measurement of a $1 \mathrm{pF}$ sensor element. Applying a least squares polynomial curve fitting can further enhance the resolution to $+/-1 \mathrm{~Hz}$ in the DSB case, and $+/-0.2 \mathrm{~Hz}$ in the SSB case. 


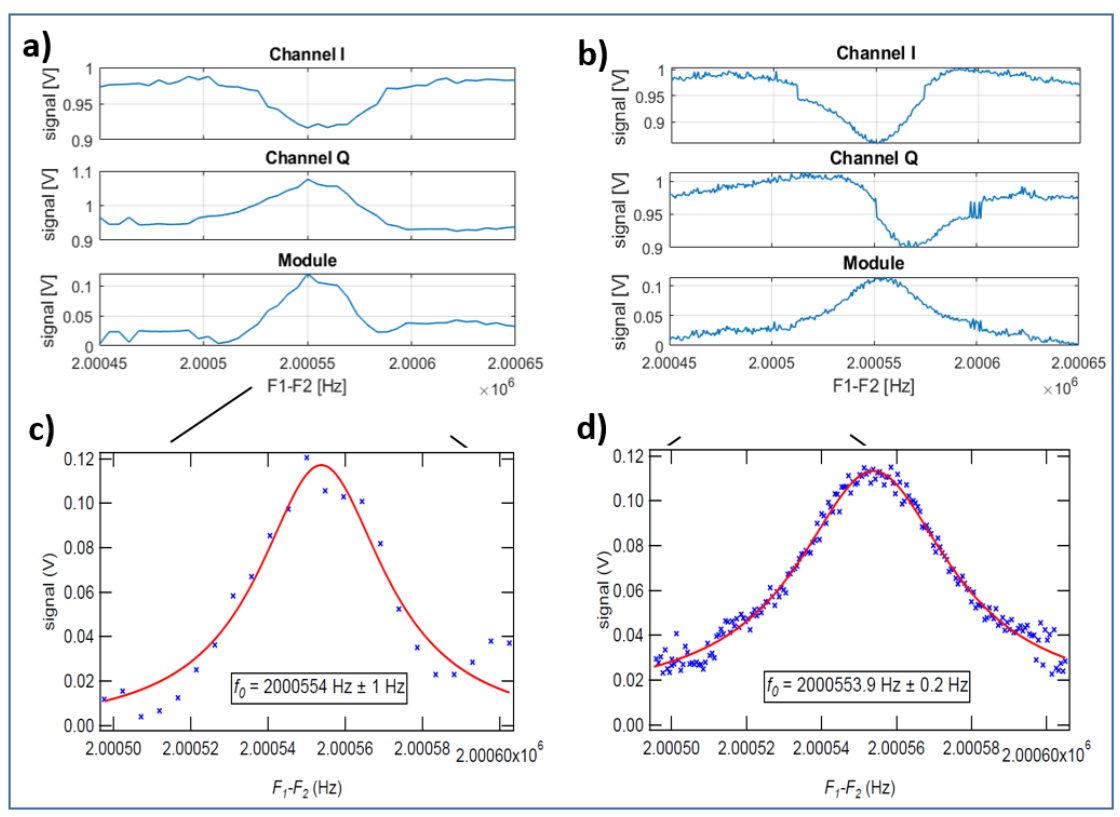

Figure 32. Evaluation of the reader frequency step resolution comparing (a) the DSB method with a minimum difference frequency step of $4.8 \mathrm{~Hz}$ and (b) the SSB method with a minimum frequency step of $0.6 \mathrm{~Hz}$. Close-up plots of the module resonance peak, with sample points marked, is given for DSB (c) and SSB (d).

The activation principle introduced in Section 3.4.5 was tested first by applying a DC bias to the diode using a benchtop DC power supply. The voltage was connected across the quartz using carefully arranged thin wires to avoid interfering with the radio signal. A sensor was placed at $2 \mathrm{~m}$ distance, and its intermodulation response was examined as the DC bias was increased from o V to $1 \mathrm{~V}$ in steps of $200 \mathrm{mV}$. The results illustrated in Figure 33a represent the $I$ channel response. The resonance peak is clearly seen with no DC bias, and as the bias increases to $600 \mathrm{mV}$ the response begins to reduce. At $1 \mathrm{~V}$ bias the sensor response is indistinguishable from the noise of the receiver. The proof of concept of the method of combining RFID and the zero power sensor was carried out using the setup illustrated in Figure 29b. The UHF RFID tag was placed orthogonal to the zero power sensor tag to minimise the coupling between them. The zero power reader and UHF RFID reader systems were kept separate, and placed a distance of $2 \mathrm{~m}$ from the sensor, with $1 \mathrm{~m}$ between their antennas. Both readers use circularly polarised antennas, so that they can each can couple to both tags. The RFID tag writing is performed first to record the address of RFID tags present and (de)activate the G2iL+ digital output, the state of which was monitored using a portable multimeter, as shown in Figure 29b. After the RFID activation was carried out the Zero Power reader was activated and began scanning for intermodulating sensor nodes. The power sent from the Zero Power reader was found to be sufficient to energize the RFID tag such as to bias the 
senor tags diode to $1.2 \mathrm{~V}$ at $2 \mathrm{~m}$ range. Figure $33 \mathrm{~b}$ shows the resonance response on the I channel when the tag is both activated and deactivated. The observed amplitude of the I channel response reduces from $180 \mathrm{mV}$ to $35 \mathrm{mV}$, giving approximately five times less signal in the deactivated state, considered sufficient to differentiate active and inactive sensors.

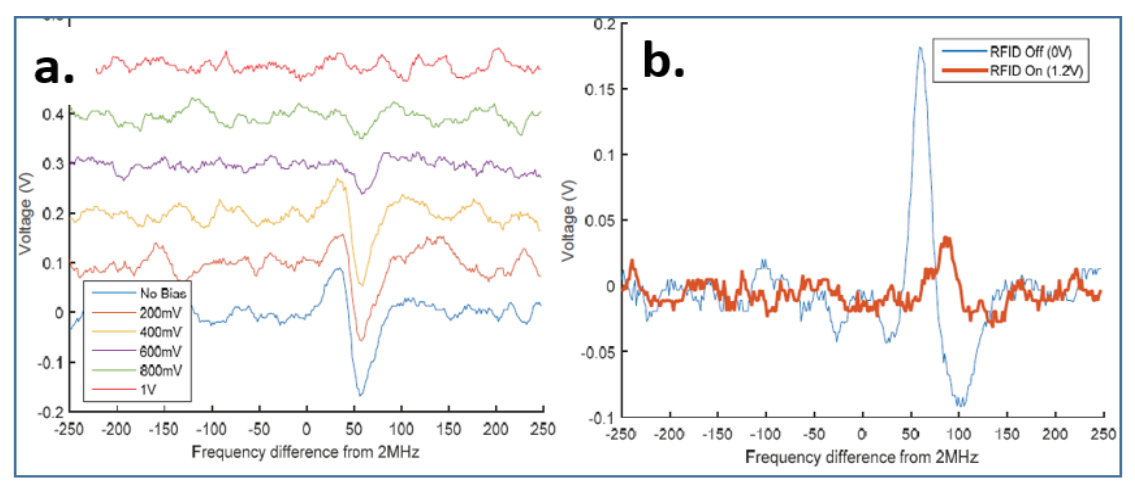

Figure 33. Results of the activation of zero power sensor using passive UHF RFID. (a) the I channel intermodulation response with reader antenna at $1 \mathrm{~m}$ distance, for a frequency sweep of $2 \mathrm{MHz} \pm 250 \mathrm{~Hz}$, with a bias voltage applied from a DC source and incremented from $0 \mathrm{~V}$ to $1 \mathrm{~V}$ in steps of $200 \mathrm{mV}$. For clarity the responses are offset in steps of $100 \mathrm{mV}$, (b) ZPS I channel intermodulation response, frequency sweep of $2 \mathrm{MHz} \pm 250 \mathrm{~Hz}$, with (red) and without (blue) a bias voltage of $1.2 \mathrm{~V}$ applied from the G2iL+ digital output, as shown in figure 2. Reader placed at a distance of $2 \mathrm{~m}$.

\subsubsection{Summary}

The work of this thesis has developed an integrated device for the readout of fully passive wireless sensors. The reader was designed based on the ad-hoc assembled reader in the previous works, integrated in a custom designed system. The reader was evaluated in terms of its two primary limitations; readout range and sensor readout resolution. Power optimisation was considered, as related directly to the maximum readout range of the system. The reader self-intermodulation noise was highlighted as the key optimisation parameter. It was reduced by applying circulator isolations between the two synthesised frequency outputs, and a maximum readout range of $16 \mathrm{~m}$ was achieved, representing a significant increase on the previous work. A technique for improving the sensor resolution was applied using a modified frequency sweeping technique which allowed a 12x increase in the frequency step resolution and therefore the sensor resolution. In addition, a concept for the combination of UHF RFID with the zero power sensor was developed and verified as a feasible method of activating and deactivating, and identifying zero power sensors. The work presented in this thesis represents significant steps forward towards the practical deployment of the zero power sensing technique in IoT applications. 



\section{Conclusion}

As IoT sensing devices become more prevalent in our world, the needs and challenges of powering them and communicating with them will continue to increase. Power autonomy and wireless communications have been highlighted in this work as two key requirements to enable the widespread deployment of IoT sensing devices. The work of this thesis has contributed four novel independent and mutually exclusive sensor systems, each interfaced wirelessly and each providing power autonomy. The power solutions presented here cover the primary options available in the context of IoT sensing devices; battery powered, energy harvester powered, wirelessly powered, and powered by far field electromagnetic radiation. This work developed radio communications in three of the most commonly used ISM frequency bands; $433 \mathrm{MHz}, 868 \mathrm{MHz}$ and $2.45 \mathrm{GHz}$. These bands include the frequency space where the vast majority of IoT devices operate, including UHF RFID, Bluetooth, Wi-Fi, ZigBee, Sigfox, LoRa and NBIoT. Additionally, the work developed wireless power transfer at 8.5 $\mathrm{MHz}$.

A swallowable capsule device, using an electronic tongue sensor, was developed for electrochemical measurements of the gastrointestinal fluids for disease diagnosis. The swallowable capsule device utilises a high energy density lithium battery to store enough energy for the maximum measurement lifetime of the device, 72 hours as defined by the maximum transit time of the human gastrointestinal tract. As the capsule is considered disposable, is it proposed to be fully power autonomous in that no energy related intervention is required during its entire measurement lifetime. Wireless communication is here considered essential to acquire the measured signal from inside the human body. It was enabled in this device using $433 \mathrm{MHz}$ transceiver system with an electrically small loop antenna inside the capsule and a dipole antenna on the external receiver module. The electrochemical E-tongue sensor developed in this work continues to be exploited for applications in food quality monitoring. The swallowable capsule device is currently being valorised through a research project in online bioreactor process monitoring.

A valve leakage detection system, using a MEMS based acoustic emission sensor, for online monitoring of petrochemical valve deterioration was developed in this work. The system presented a particular challenge in the monitoring of valve deterioration leading to leakage. As this occurs on the time scales of months and years, measurements need only be taken at long intervals, in the 
order of one day. Power autonomy was desired for continuous and indefinite operation in both the sensor nodes and for the reader gateway. To achieve power autonomy, the design integrated an ultra-low power wake-up radio which allowed the sensor nodes to be put in a very low power sleep mode and asynchronously interrogated on demand by the reader. Energy autonomy was achieved in the sensor nodes by applying a thermal energy harvester to exploit the temperature difference between the valves petrochemical payload and the ambient environment. In the reader gateway energy autonomy was achieved using an industrial current loop energy harvester. Wireless communication is here considered essential as a dangerous industrial environment is incompatible with retrofitted wired cables. The wake-up radio was implemented using a low frequency signal modulated on a $2.45 \mathrm{GHz}$ carrier and active data communication was implemented at $2.45 \mathrm{GHz}$. Quarter wave monopole antennas were used on both the sensor node and receiver gateway. The valve leakage detection system was deployed and piloted at Metso Flow Control Oy. The acoustic emission sensor continues to be exploited in other online condition monitoring measurements and the ultra-low power wake-up radio is available for exploitation in a wide range of applications.

A wirelessly powered soft robotic caterpillar was developed in this work. The device applied resonant inductive coupling, and a frequency sweeping technique to transfer power to multiple shape memory alloy actuators tuned at different resonant frequencies. The robotic caterpillar itself is fully passive and all power is delivered wirelessly from a transmitter coil. Control of the robots movement is achieved by applying two separate receiver coils on the robot, tuned at different resonant frequencies and transmitting power either by frequency sweeping or by sending power pulses at differing frequencies. The robotic caterpillar manifested was purely an actuator device, however it may be considered a sensor platform where sensor elements can be added and configured to exploit the inductively coupled energy for sensing and communication functions. The soft robotic caterpillar continues to advance, and now includes, for example, a more complex device for climbing. Further development in the work of this thesis may include the investigation of far field power transfer, and the expansion of the number of receiver-actuators to enable more complex movements of the caterpillar.

Finally, the work of this thesis has contributed towards the development of the zero power sensing system. In this case, power autonomy is enabled by utilising fully passive sensor nodes which receive and reflect all the needed energy for sensing from the reader device. The sensor utilised third order intermodulation to down convert radio signals, and a loaded high-Q resonator for the sensing technique. Therefore, radio powering and communication is integrated in the same incident signal at UHF frequencies (868 MHz). This work focused on the development of a reader device, its evaluation and optimisation to enhance the readout range and sensing resolution of the passive sensors. In addition, this 
work tackles the inherent single channel nature of the zero power system by integrating the sensor with UHF RFID for activation and identification of sensor nodes. The zero power sensor system continues to be exploited in a wide range of applications including temperature, pressure, inclination and acceleration monitoring. Further developments can include deeper integration of the reader device and fusion of RFID technology with the sensor tag.

This thesis highlights that there is no universal solution for achieving power autonomous wireless sensors. Instead, a system level approach is required and this has been the approach taken in each of the described cases. As emphasized in the thesis, the radio and powering methodologies are integral in the complex development of power autonomous wireless sensors. This can be observed most clearly in the valve leakage detection system, where the ultra-low power wakeup radio is the core technology enabling power autonomy, or the zero power passive sensor where the radio waves provide energy for the sensing, and carry the sensor measurement. In the case of the robotic caterpillar, the radio waves carry the power from the transmitter to the device. On the other hand, in the battery powered capsule, the radio system is purely a draw on the energy supply, but nonetheless essential for communication of the measured data. In all of the systems developed in this work, the radio communication technology is emphasized as one of the most significant system level considerations in the quest for power autonomy.

This work has explored the challenge of power autonomy in the context of wireless sensing IoT devices. Examining four independent cases, this work has illustrated that a holistic system level approach to power autonomy is required. This work has exploited four of the most practical powering mechanisms available to IoT system developers; battery power, energy harvesting, wirelessly powered, and by far field radio backscattering. The work has exploited communication systems at $433 \mathrm{MHz}, 868 \mathrm{MHz}$ and $2.45 \mathrm{GHz}$, and investigated ultralow power wake-up radio and a passive back scattering sensor based on third order intermodulation. This thesis has presented four very different approaches to the delivery a measurement system that can perform fully its required functions without power related intervention by the user. The impact of this work as a whole is to provide a number of solutions that enhance the feasibility of the deployment of IoT sensors for indefinite operation without energy related intervention from the user. This is a key advancement towards the wide adoption of IoT sensors in our world 



\section{References}

[1] M. Bellevilleet al, "Energy autonomous sensor systems: Towards a ubiquitous sensor technology," Microelectronics Journal, vol. 41, no.11, pp. 740-745, 2010.

[2] Rook Security. "IoT device category guide," 2017. [Online]. Available: https://www.rooksecurity.com/building-security-into-iot-development/iot-devicecategory-guide/. [Accessed: Nov. 30, 2018].

[3] Statista. "Internet of things (IoT) did-27915-1," 2018. [Online]. Available: https://www.statista.com/study/27915/internet-of-things-iot-statista-dossier/. [Accessed: Nov. 30, 2018].

[4] C. McCaffrey et al, "Live demonstration: Energy autonomous wireless valve leakage monitoring system with acoustic emission sensor," in IEEE Sensors 2017, pp. 1.

[5] Abhi Raj and Dan Steingart, "Review-Power sources for the internet of things," Journal of the Electrochemical Society, vol. 165, no. 8, pp. 3130-3136, 2018.

[6] B. Martinez et al, "The power of models: Modeling power consumption for loT devices," IEEE Sensors Journal, vol. 15, no. 10, pp. 5777-5789, 2015.

[7] Z. Abbas and W. Yoon, "A survey on energy conserving mechanisms for the internet of things: Wireless networking aspects," Sensors, vol. 15, no. 10, pp. 24818-

24847, 2015.

[8] H. Yetgin et al, "A survey of network lifetime maximization techniques in wireless sensor networks," IEEE Communications Surveys \& Tutorials, vol. 19, no. 2, pp. 828854, 2017.

[9] Yanwei Wu et al, "Energy-efficient wake-up scheduling for data collection and aggregation," IEEE Transactions on Parallel and Distributed Systems, vol. 21, no. 2, pp. 275-287, 2010.

[10] A. Dementyev et al, "Power consumption analysis of bluetooth low energy, ZigBee and ANT sensor nodes in a cyclic sleep scenario," in IEEE International Wireless Symposium (IWS) 2013, pp. 1-4.

[11] CYPRESS. "PSoC ${ }^{\circledR} 4$ : PSoC 4XX7_BLE family datasheet programmable system-onchip (PSoC ${ }^{\circledast}$ ) - download," 2017. [Online]. Available: http://www.cypress.com/file/137466/download. [Accessed: Feb. 19, 2018]. 
[12] Texas Instruments. "2.4-GHz bluetooth low energy and proprietary system-onchip. AN-CC2541," 2017. [Online]. Available: http://www.ti.com/lit/ds/symlink/cc2541.pdf. [Accessed: Nov. 30, 2018].

[13] Cypress. "AN92584 - designing for low power and estimating battery life for BLE applications," 2017. [Online]. Available: http://www.cypress.com/AN92584. [Accessed: Nov. 30, 2018].

[14] Texas Instruments. "AN092 -- measuring bluetooth low energy power consumption (rev. A)," 2012. [Online]. Available:

http://www.ti.com/lit/an/swra347a/swra347a.pdf. [Accessed: Nov. 30, 2018].

[15] Nordic Semiconductor. "nRF52832 product specification v1.4,". [Online]. Available: http://infocenter.nordicsemi.com/pdf/nRF52832 PS v1.4.pdf. [Accessed: Jan. 30, 2018].

[16] V. Jelicic et al, "Analytic comparison of wake-up receivers for WSNs and benefits over the wake-on radio scheme," in Proceedings of the 7th ACM workshop on performance monitoring and measurement of heterogeneous wireless and wired networks Oct 21, 2012, pp. 99-106.

[17] G.U. Gamm et al, "Low power wake-up receiver for wireless sensor nodes," in 2010 Sixth International Conference on Intelligent Sensors, Sensor Networks and Information Processing 2010, pp. 121-126.

[18] S.J. Marinkovic and E.M. Popovici, "Nano-power wireless wake-up receiver with serial peripheral interface," IEEE Journal on Selected Areas in Communications, vol. 29, no. 8, pp. 1641-1647, 2011.

[19] EM Microelectronic. "1 kbit read/write, EPC Gen2 / ISO 18000-63 type C passive or battery-assisted passive RFID IC, EM4324 datasheet," 2017. [Online]. Available: http://www.emmicroelectronic.com/sites/default/files/products/datasheets/em4324 ds.pdf. [Accessed: Feb, 16, 2018].

[20] P. Pursula, V. Viikari, and J. Saari, "Wake-up radio architecture utilizing passive down conversion mixing," Microwave and Optical Technology Letters, vol. 55, no. 5, pp. 1038-1041, 2013.

[21] C. Knight, J. Davidson, and S. Behrens, "Energy options for wireless sensor nodes," Sensors, vol. 8, no. 12, pp. 8037-8066, 2008.

[22] J.F.M. Oudenhoven, R.J.M. Vullers, and R. Schaijk, "A review of the present situation and future developments of micro-batteries for wireless autonomous sensor systems," International Journal of Energy Research, vol. 36, no. 12, pp. 1139-1150, 2012.

[23] B. Scrosati and J. Garche, "Lithium batteries: Status, prospects and future," Journal of Power Sources, vol. 195, no. 9, pp. 2419-2430, 2010.

[24] J. Ordoñez, E.J. Gago, and A. Girard, "Processes and technologies for the recycling and recovery of spent lithium-ion batteries," Renewable and Sustainable Energy Reviews, vol. 60, pp. 195-205, 2016. 
[25] J.A. Paradiso and T. Starner, "Energy scavenging for mobile and wireless electronics," IEEE Pervasive Computing, vol. 4, no. 1, pp. 18-27, 2005.

[26] R. Vullers et al, "Energy harvesting for autonomous wireless sensor networks," IEEE Solid-State Circuits Magazine, vol. 2, no. 2, pp. 29-38, 2010.

[27] Chin Keong Ho and Rui Zhang, "Optimal energy allocation for wireless communications with energy harvesting constraints," IEEE Transactions on Signal Processing, vol. 60, no. 9, pp. 4808-4818, 2012.

[28] F.K. Shaikh and S. Zeadally, "Energy harvesting in wireless sensor networks: A comprehensive review," Renewable and Sustainable Energy Reviews, vol. 55, pp. 1041-1054, 2016.

[29] Anonymous, "Silicon cells harvest sun's energy," Electrical Engineering, vol. 77, no. 11, pp. 1073-1074, 1958.

[30] B. Subudhi and R. Pradhan, "A comparative study on maximum power point tracking techniques for photovoltaic power systems," IEEE Transactions on Sustainable Energy, vol. 4, no. 1, pp. 89-98, 2013.

[31] Vijay Raghunathan et al, "Design considerations for solar energy harvesting wireless embedded systems," in IPSN 2005. Fourth International Symposium on Information Processing in Sensor Networks 2005, pp. 457-462.

[32] D. Brunelli et al, "Design of a solar-harvesting circuit for batteryless embedded systems," IEEE Transactions on Circuits and Systems I: Regular Papers, vol. 56, no. 11, pp. 2519-2528, 2009.

[33] S. Lineykin and S. Ben-Yaakov, "Modeling and analysis of thermoelectric modules," IEEE Transactions on Industry Applications, vol. 43, no. 2, pp. 505-512, 2007.

[34] V. Leonov, "Thermoelectric energy harvesting of human body heat for wearable sensors," IEEE Sensors Journal, vol. 13, no. 6, pp. 2284-2291, 2013.

[35] Xin Lu and Shuang-Hua Yang, "Thermal energy harvesting for WSNs," in 2010 IEEE International Conference on Systems, Man and Cybernetics 2010, pp. 30453052.

[36] J.P. Carmo, L.M. Goncalves, and J.H. Correia, "Thermoelectric microconverter for energy harvesting systems," IEEE Transactions on Industrial Electronics, vol. 57, no. 3, pp. 861-867, 2010.

[37] P.D. Mitcheson et al, "Energy harvesting from human and machine motion for wireless electronic devices," Proceedings of the IEEE, vol. 96, no. 9, pp. 1457-1486, 2008.

[38] S. Chamanian et al, "Wearable battery-less wireless sensor network with electromagnetic energy harvesting system," Sensors and Actuators A: Physical, vol. 249, pp. 77-84, 2016. 
[39] Xiao Lu et al, "Wireless networks with RF energy harvesting: A contemporary survey," IEEE Communications Surveys \& Tutorials, vol. 17, no. 2, pp. 757-789, 2015.

[40] C.R. Valenta and G.D. Durgin, "Harvesting wireless power: Survey of energy-harvester conversion efficiency in far-field, wireless power transfer systems," IEEE Microwave Magazine, vol. 15, no.4, pp. 108-120, 2014.

[41] Sangkil Kim et al, "Ambient RF energy-harvesting technologies for self-sustainable standalone wireless sensor platforms," Proceedings of the IEEE, vol. 102, no. 11, pp. 1649-1666, 2014.

[42] N. Tesla, "The transmission of electrical energy without wires," Electrical World and Engineer, vol. XLIII, pp. 429-431, 1904.

[43] A. Kurs et al, "Wireless power transfer via strongly coupled magnetic resonances," Science, vol. 317, no. 5834, pp. 83-86, 2007.

[44] N. Shinohara, "Power without wires," IEEE Microwave Magazine, vol. 12, no.7, pp. S73, 2011.

[45] S.Y.R. Hui, W. Zhong, and C.K. Lee, "A critical review of recent progress in midrange wireless power transfer," IEEE Transactions on Power Electronics, vol. 29, no. 9, pp. 4500-4511, 2014.

[46] R. Zhang and C. K. Ho, "MIMO broadcasting for simultaneous wireless information and power transfer," IEEE Transactions on Wireless Communications, vol. 12, no. 5, pp. 1989-2001, 2013.

[47] D. Patil et al, "Wireless power transfer for vehicular applications: Overview and challenges," IEEE Transactions on Transportation Electrification, vol. 4, no. 1, pp. 337, 2018.

[48] S.Y. Hui, "Planar wireless charging technology for portable electronic products and qi," Proceedings of the IEEE, vol. 101, no. 6, pp. 1290-1301, 2013.

[49] K.N. Bocan and E. Sejdić, "Adaptive transcutaneous power transfer to implantable devices: A state of the art review," Sensors, vol. 16, no. 3, pp. 393, 2016.

[50] X. Lu et al, "Wireless charging technologies: Fundamentals, standards, and network applications," IEEE Communications Surveys \& Tutorials, vol. 18, no. 2, pp. 14131452, 2016.

[51] F. Michahelles et al, "Pervasive RFID and near field communication technology," IEEE Pervasive Computing, vol. 6, no. 3, pp. 96, c3, 2007.

[52] Wireless Power Consortium. "Qi standard version 1.2.3," 2016. [Online]. Available: https://www.wirelesspowerconsortium.com/downloads/download-wirelesspower-specification.html. [Accessed: Feb. 22, 2018]. 
[53] T. Deyle and M. Reynolds, "Surface based wireless power transmission and bidirectional communication for autonomous robot swarms," in 2008 IEEE International Conference on Robotics and Automation 2008, pp. 1036-1041.

[54] M. Karpelson et al, "A wirelessly powered, biologically inspired ambulatory microrobot," in 2014 IEEE International Conference on Robotics and Automation (ICRA) 2014, pp. 2384-2391.

[55] M.S. Mohamed Ali and K. Takahata, "Wireless microfluidic control with integrated shape-memory-alloy actuators operated by field frequency modulation," Journal of Micromechanics and Microengineering, vol. 21, pp. 075005, 2011.

[56] M. Boyvat, J. Koh, and R.J. Wood, "Addressable wireless actuation for multijoint folding robots and devices," Science Robotics, vol. 2, no. 8, 2017.

[57] Y. Kim et al, "An earthworm-like micro robot using shape memory alloy actuator," Sensors \& Actuators: A. Physical, vol. 125, no. 2, pp. 429-437, 2006.

[58] T. Umedachi, V. Vikas, and B.A. Trimmer, "Highly deformable 3-D printed soft robot generating inching and crawling locomotions with variable friction legs," in IEEE/RSJ International Conference on Intelligent Robots and Systems 2013, pp. 45904595.

[59] J. Song, "On passive wireless sensors based on intermodulation communication ", Doctoral Dissertation, Dept. Radio Sci. \& Eng., Aalto University, 2015.

[60] Bo, Lui., Xiao, Chen., Hualin, Cai, Ali, Mohammad, Xangguang, Tian, Luqi, Tao. Yi, Yang and Tianling, Ren, "Surface acoustic wave devices for sensor applications," Journal of Semiconductors, vol. 37, no. 2, pp. 1-9, 2016.

[61] A. Pohl, "A review of wireless SAW sensors," IEEE transactions on ultrasonics, ferroelectrics, and frequency control, vol. 47, no. 2, pp. 317-332, 2000.

[62] C.S. Hartmann and L.T. Claiborne, "Fundamental limitations on reading range of passive IC-based RFID and SAW-based RFID," in 2007 IEEE International Conference on RFID 2007, pp. 41-48.

[63] F. Nawaz and V. Jeoti, "SAW sensor read range limitations and perspectives," Wireless Networks, vol. 20, no. 8, pp. 2581-2587, 2014.

[64] GS1. "EPC compliant class-1 generation-2 UHF RFID devices conformance requirements 2.0.1," 2015. [Online]. Available:

https://www.gs1.org/docs/epc/Gen2 conformance requirements.pdf. [Accessed: Feb. 28, 2018].

[65] R. Weinstein, "RFID: A technical overview and its application to the enterprise," IT Professional, vol. 7, no. 3, pp. 27-33, 2005.

[66] P. V. Nikitin and K. V. S. Rao, "Theory and measurement of backscattering from RFID tags," IEEE Antennas and Propagation Magazine, vol. 48, no. 6, pp. 212-218, 2006. 
[67] S. Roy et al, "RFID: From supply chains to sensor nets," Proceedings of the IEEE, vol. 98, no. 9, pp. 1583-1592, 2010.

[68] D. J. Yeager et al, "Wirelessly-charged UHF tags for sensor data collection," in IEEE International Conference on RFID 2008, pp. 320-327.

[69] A. P. Sample et al, "Design of an RFID-based battery-free programmable sensing platform," IEEE Transactions on Instrumentation and Measurement, vol. 57, no. 11, pp. 2608-2615, 2008.

[70] A. Vaz et al, "Full passive UHF tag with a temperature sensor suitable for human body temperature monitoring," IEEE Transactions on Circuits and Systems II: Express Briefs, vol. 57, no. 2, pp. 95-99, 2010.

[71] H. Reinisch et al, "A multifrequency passive sensing tag with on-chip temperature sensor and off-chip sensor interface using EPC HF and UHF RFID technology," IEEE Journal of Solid-State Circuits, vol. 46, no. 12, pp. 3075-3088, 2011.

[72] J. M. Collignon et al, "RF energy harvester optimized for wireless sensor network in launcher application," 2017, pp. 48-51.

[73] M. M. Islam et al, "UHF RFID sensors based on frequency modulation," in IEEE Sensors 2016, pp. 1-3.

[74] C. Occhiuzzi, S. Caizzone, and G. Marrocco, "Passive UHF RFID antennas for sensing applications: Principles, methods, and classifcations," IEEE Antennas and Propagation Magazine, vol. 55, no. 6, pp. 14-34, 2013.

[75] J. G. Vogler, D. J. Maquire, and A. E. Steinhauer, "DINADE-A new interrogation, navigation and detection system," Microwave Journal, vol. 10, no. 4, pp. 2-6, 1967.

[76] K. Rasilainen et al, "On design and evaluation of harmonic transponders," IEEE Transactions on Antennas and Propagation, vol. 63, no. 1, pp. 15-23, 2015.

[77] J. Song et al, "On the use of the intermodulation communication towards zero power sensor nodes," in Proceedings of European Microwave Association 2013, pp. 124-127.

[78] R.S. Mackay and B. Jacobson, "Endoradiosonde," Nature, vol. 179, pp. 1239, 1957.

[79] G. Ciuti, A. Menciassi, and P. Dario, "Capsule endoscopy: From current achievements to open challenges," IEEE Reviews in Biomedical Engineering, vol. 4, pp. 59-72, 2011.

[80] T. D. Than et al, "A review of localization systems for robotic endoscopic capsules," IEEE Transactions on Biomedical Engineering, vol. 59, no. 9, pp. 2387-2399, 2012. 
[81] Y. T. Jui et al, "The ESO-pill: A non-invasive MEMS capsule for bolus transit monitoring in the esophagus," in Proceedings of the 2004 11th IEEE International Conference on Electronics, Circuits and Systems 2004, pp. 427-430.

[82] Lei Wang et al, "A programmable microsystem using system-on-chip for realtime biotelemetry," IEEE Transactions on Biomedical Engineering, vol. 52, no. 7, pp. 1251-1260, 2005.

[83] K. Kalantar-zadeh et al, "Ingestible sensors," ACS Sensors, vol. 2, no. 4, pp. 468483, 2017.

[84] K. Twomey, A. Truemper, and K. Murphy, "A portable sensing system for electronic tongue operations," Sensors, vol. 6, no. 11, pp. 1679-1696, 2006.

[85] P. Jesudoss et al, "System packaging \& integration for a swallowable capsule using a direct access sensor," in 2009 European Microelectronics and Packaging Conference 2009, pp. 1-4.

[86] P. Jesudoss, "Development and reliability of a direct access sensor using flip chip on flex technology with anisotropic conductive adhesive", PhD. Dissertation, University College Cork, Dept. Elec. \& Electronic Eng., 2011.

[87] J. Halme, T. Sillanpää, P. Pekko, M. Heikkinen, A. Oja, "Micromechanical acoustic emmision sensors for condition monitoring," in Proc. 16th Eur. Conf. Solid-State Transducers Transducers, Eurosensors 2002, pp. 328-332.

[88] P. Pursula, V. Viikari, and J. Saari, "Wake-up radio architecture utilizing passive down conversion mixing," Microwave and Optical Technology Letters, vol. 55, no. 5, pp. 1038-1041, 2013.

[89] T. Jespen, ATEX - explosive atmospheres : Risk assessment, control and compliance, Springer, Cham, 2016.

[90] Jin Yan et al, "Nondestructive detection of valves using acoustic emission technique," Advances in Materials Science and Engineering, vol. 2015, pp. 1-9, 2015.

[91] Colm Mc Caffrey. "DEMO 6: Live demonstration: Energy autonomous wireless valve leakage monitoring system with acoustic emission sensor," 2017. [Online]. Available: http://ieee-sensors2017.org/live-demos. [Accessed: Feb. 20, 2019].

[92] E. Waffenschmidt, "Homogeneous magnetic coupling for free positioning in an inductive wireless power system," IEEE Journal of Emerging and Selected Topics in Power Electronics, vol. 3, no. 1, pp. 226-233, 2015.

[93] V. Viikari, H. Seppä, and K. Dong-Wook, "Intermodulation read-out principle for passive wireless sensors," IEEE Transactions on Microwave Theory and Techniques Vol.59 Nr. 4, pt. 1, 1025-1031, 2011.

[94] J. Song et al, "Realizing frequency division multiple access with passive wireless intermodulation communication sensors," Microwave and Optical Technology Letters, vol. 57, no. 2, pp. 274-277, 2015. 
This thesis examines power autonomy in wireless sensors. Four independent systems are presented, each a power autonomous solution. The first system integrates a voltammetric electronic tongue sensor for gastrointestinal disease diagnosis, with power provided by a high density lithium manganese dioxide primary cell. The second system applies a MEMS acoustic emission sensor for online condition monitoring of valve leakage, utilising thermal energy harvesting in the sensor node, and industrial current loop harvesting in the receiver. The third system exploits resonant inductive coupling for the actuation of a wirelessly powered soft robotic caterpillar which itself is potentially a sensor platform. The fourth system employs a fully passive wireless sensing platform based on radio backscattering, and a harmonic resonant sensor based on the third order intermodulation principle.

This work represents progress on multiple fronts on the key challenge of powering the next generation of IoT devices.

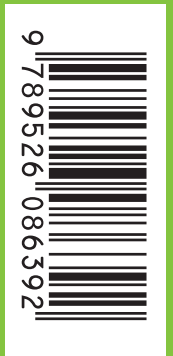

